ESAIM: COCV 27 (2021) 95

https://doi.org/10.1051/cocv/2021093
ESAIM: Control, Optimisation and Calculus of Variations

www.esaim-cocv.org

\title{
CUBIC MICROLATTICES EMBEDDED IN NEMATIC LIQUID CRYSTALS: A LANDAU-DE GENNES STUDY
}

\author{
Razvan-Dumitru CeucA ${ }^{1,2,3, *}$ ๑
}

\begin{abstract}
We consider a Landau-de Gennes model for a connected cubic lattice scaffold in a nematic host, in a dilute regime. We analyse the homogenised limit for both cases in which the lattice of embedded particles presents or not cubic symmetry and then we compute the free effective energy of the composite material. In the cubic symmetry case, we impose different types of surface anchoring energy densities, such as quartic, Rapini-Papoular or more general versions, and, in this case, we show that we can tune any coefficient from the corresponding bulk potential, especially the phase transition temperature. In the case with loss of cubic symmetry, we prove similar results in which the effective free energy functional has now an additional term, which describes a change in the preferred alignment of the liquid crystal particles inside the domain. Moreover, we compute the rate of convergence for how fast the surface energies converge to the homogenised one and also for how fast the minimisers of the free energies tend to the minimiser of the homogenised free energy.
\end{abstract}

Mathematics Subject Classification. 35J50, 35B27, 76M50, 76A15.

Received August 10, 2020. Accepted September 22, 2021.

\section{INTRODUCTION}

We consider a cubic microlattice scaffold constructed of connected particles of micrometer scale, within a nematic liquid crystal. In this article, we treat the particles of the cubic microlattice as being inclusions from the mathematical point of view, while they might be interpreted as colloids from the physical point of view, even though they do not possess all of their properties. The cubic microlattice scaffold is also called a bicontinuous porous solid matrix (BPSM) in the physics literature (for example, see [7, 23] or [24]). By cubic microlattice scaffold we understand a connected family of parallelepipeds or cubes of different sizes, placed in a periodic fashion, as in Figure 1, where only the embedded particles have been shown. For simplicity, we might refer to this object as being a scaffold or a cubic microlattice. This type of scaffold is usually obtained using the two-photon polymerization (TPP or $2 \mathrm{PP}$ ) process, which represents a technique of $3 \mathrm{D}$-manufacturing structures and which can generate stand-alone objects. An overview of the field of TPP processes can be found in [4]. There are numerous experiments, theory and computer simulations regarding embedding microparticles into nematic liquid crystals (for example, see [18, 21, 22]).

Keywords and phrases: Liquid crystals, Landau-de Gennes, Gamma-convergence, Cubic microlattices.

${ }^{1}$ BCAM, Basque Center for Applied Mathematics, Mazarredo 14, 48009 Bilbao, Bizkaia, Spain.

2 UPV/EHU, Universidad del País Vasco/Euskal Herriko Unibertsitatea, Barrio Sarriena s/n, 48940 Leioa, Bizkaia, Spain.

3 UAIC, Alexandru Ioan Cuza University of Iasi, Bulevardul Carol I, Nr. 11, 700506 Iasi, Romania.

* Corresponding author: rceuca@bcamath.org 


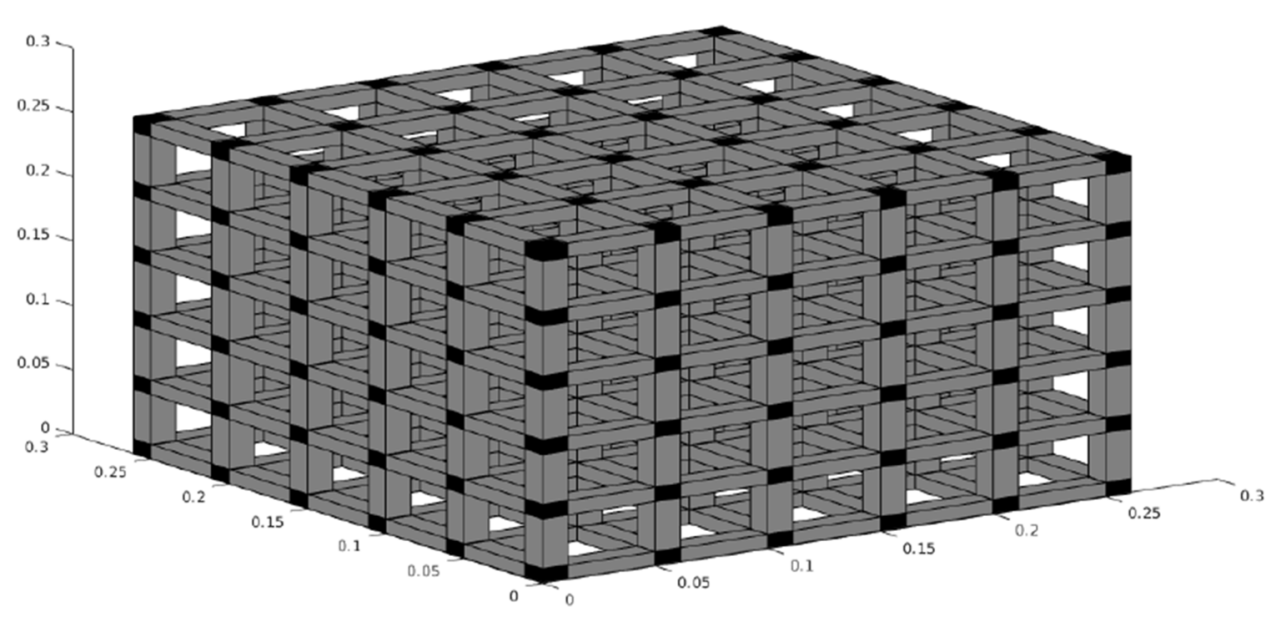

Figure 1. Example of a cubic microlattice.

The system bears mathematical similarities to that of colloids embedded into nematic liquid crystals. The mathematical studies of nematic colloids (the mixture of colloidal particles embedded into nematic liquid crystals) are split into two broad categories:

- one is dealing with the effect produced by a small number of particles in this mixture, with a focus on the defect patterns that arise in the alignment of the nematic particles induced by the interaction at the boundary of the colloid between the two combined materials (see, for example, [2, 3, 9-12, 25]);

- the other one treats the study of the collective effects, that is the homogenisation process (see, for example, $[5,6,8,13,14])$.

This work continues within the second direction, that is studying the homogenised material, and it is built on the work from $[13,14]$, which was also based on $[5,6,8]$. The general thrust of these papers is to prove that the homogenisation limit of a nematic liquid crystal with colloidal inclusions of a specific geometry can generate a new material, which behaves like a new nematic liquid crystal, but now with different material parameters.

In $[13,14]$, the set of inclusion particles is disconnected, obtained from different or identical model particles, in such a way that the distance between the particles is considerable larger than the size of them, which is called the dilute regime. Also, in this regime, the volume fraction of colloids tends to zero.

In this article, we are going to consider the case of a cubic microlattice scaffold, as shown in Figure 1. The idea of using such a particular geometry for the scaffold comes from the work done in [23]. At the same time, this geometric configuration is more relevant from the physical point of view, since in $[13,14]$ one cannot position a priori the colloidal particles in a periodic fashion. Here the periodicity is automatically generated by the structure of the cubic microlattice. We construct two types of scaffolds: one with identical cubes centered in a periodic 3D lattice of points, cubes which are inter-connected by parallelepipeds, and one where we replace the cube with a parallelepiped with three different length sides. If by cubic symmetry we understand the family of rotations that leave a cube invariant, then the first case is when the scaffold particles have cubic symmetry and the second one is with the loss of this type of symmetry.

The main new aspects of this work are:

- the set of all the inclusion particles is now replaced with an individual inclusion particle, which can be seen as a connected union of smaller particles

- the model particle that we use (that is, a parallelepiped or a cube) grants us the possibility to compute the surface contribution for arbitrarily high order terms in the surface energy density - hence, a generalisation has been done for higher order polynomials in the bulk energy potential that admit at least one local minimiser (see Thm. 3.6); 
- in the case where the cubic symmetry is lost, we obtain a new term into the homogenised limit that can be seen as a change in the preferred alignment of the liquid crystal particles inside the domain (see Thm. 3.7);

- we obtain a rate of convergence for how fast the surface energies converge to the homogenised one (more details in Prop. 6.4); in Remark 6.5, we also obtain a rate of convergence for how fast the sequence of minimisers of the free energies tend to a minimiser of the homogenised free energy.

Liquid crystal materials, which typically consist of either rod-like or disc-like molecules, can achieve a state of matter which has properties between those of conventional liquids and those of solid crystals. The liquid crystal state of matter is one where there exists a long range orientational order for the molecules. In order to quantify the local preferred alignment of the rod-like molecules, we use the theory of Q-tensors (for more details, see [20]). A background of the field of liquid crystal materials can be found in [16].

Let $\Omega \subset \mathbb{R}^{3}$ be an open and bounded domain from $\mathbb{R}^{3}$. For every $\varepsilon>0$, we construct a cubic microlattice $\mathcal{N}_{\varepsilon}$ inside of $\Omega$, such that, as $\varepsilon \rightarrow 0$, the volume of the scaffold tends to 0. More details regarding the construction of the cubic microlattice can be found in Section 2 and in Appendix A.1.

Let $\Omega_{\varepsilon}=\Omega \backslash \mathcal{N}_{\varepsilon}$, which represents the space where only liquid crystal particles can be found. We use functions $Q: \Omega_{\varepsilon} \rightarrow \mathcal{S}_{0}$ to describe the orientation of the liquid crystal particles, where:

$$
\mathcal{S}_{0}=\left\{Q \in \mathbb{R}^{3 \times 3}: Q=Q^{T}, \operatorname{tr}(Q)=0\right\},
$$

is denoted as the set of $Q$-tensors. In the space $\mathcal{S}_{0}$, if we define $|Q|=\left(\operatorname{tr}\left(Q^{2}\right)\right)^{1 / 2}$, for any $Q \in \mathcal{S}_{0}$, we can see that $\mathcal{S}_{0}$ is a normed linear space and the so-called Frobenius norm is induced by the scalar product $Q \cdot P=\operatorname{tr}(Q \cdot P)$.

We consider the following Landau-de Gennes free energy functional:

$$
\mathcal{F}_{\varepsilon}[Q]:=\int_{\Omega_{\varepsilon}}\left(f_{e}(\nabla Q)+f_{b}(Q)\right) \mathrm{d} x+\frac{\varepsilon^{3}}{\varepsilon^{\alpha}\left(\varepsilon-\varepsilon^{\alpha}\right)} \int_{\partial \mathcal{N}_{\varepsilon}} f_{s}(Q, \nu) \mathrm{d} \sigma
$$

where $f_{e}$ represents the elastic energy, $f_{b}$ the bulk energy, $f_{s}$ the surface density energy, $\alpha$ is a real parameter and $\partial \mathcal{N}_{\varepsilon}$ the surface of the scaffold. The coefficient in front of the surface energy term is chosen such that the denominator $\varepsilon^{\alpha}\left(\varepsilon-\varepsilon^{\alpha}\right)$ balances the effect given by the surface terms from $\partial \mathcal{N}_{\varepsilon}$, in the limit $\varepsilon \rightarrow 0$.

The elastic energy, also called the distortion energy, penalises the distortion of $Q$ in the space and, in the Landau-de Gennes theory, it is usually considered to be a positive definite quadratic form in $\nabla Q$. More details regarding the elastic energy used can be found in Section 3.2.

The bulk energy in our case consists only of the thermotropic energy, which is a potential function that describes the preferred state of the liquid crystal, that is either uniaxial, biaxial or isotropic ${ }^{1}$. For large values of the temperature, the minimum of this energy is obtained in the isotropic case, that is $Q=0$, and for small values, the minimum set is a connected set of the form $s\left(\nu \otimes \nu-\mathbb{I}_{3} / 3\right)$, with $\nu \in \mathbb{S}^{2}$ and $\mathbb{I}_{3}$ the identity $3 \times 3$ matrix, and this is a connected set diffeomorphic with the real projective plane. The simplest form that we can take for the bulk energy in our case is the quartic expansion:

$$
f_{b}(Q)=a \operatorname{tr}\left(Q^{2}\right)-b \operatorname{tr}\left(Q^{3}\right)+c \operatorname{tr}\left(Q^{2}\right)^{2}
$$

where the coefficient $a$ depends on the temperature of the liquid crystal and $b$ and $c$ depend on the properties of the liquid crystal material, with $b, c>0$. The coefficient of $\operatorname{tr}\left(Q^{2}\right)$ depends on the temperature at which the phase transition occurs. More specifically, $a$ from (1.2) is of the form $a:=a_{*}\left(T-T_{*}\right)$, in which $a_{*}$ is a material parameter and $T_{*}$ is the characteristic temperature of the nematic liquid crystal material (the temperature where the isotropic state starts losing local stability). More details regarding the bulk energy used can be found in Section 3.2.

\footnotetext{
${ }^{1}$ The isotropic case corresponds to the case in which $Q=0$. The uniaxial case corresponds to the one in which two of the eigenvalues of $Q$ are equal and the third one has a different value. The biaxial case corresponds to the case in which all the eigenvalues have different values.
} 
The surface energy describes the interaction between the liquid crystal material and the boundary of the scaffold. We assume, for simplicity, that it depends only on $Q$ and on $\nu$, where $\nu$ is the outward normal at the boundary of the cubic microlattice. Throughout this work, we choose several versions for the surface energy, depending on the bulk energy used and on whether the scaffold presents cubic symmetry or not. More details regarding the surface energies used can be found in Section 3.2.

We are interested in studying the behaviour of the whole material when $\varepsilon \rightarrow 0$. We will show that in our dilute regime we obtain for the homogenised material an energy functional of the following form

$$
\mathcal{F}_{0}[Q]:=\int_{\Omega}\left(f_{e}(\nabla Q)+f_{b}(Q)+f_{h o m}(Q)\right) \mathrm{d} x
$$

where $f_{\text {hom }}$ is defined in (3.1) and in (3.2), depending on the choice of $f_{b}$.

Our focus will be on a priori designing the $f_{\text {hom }}$, in terms of the available parameters of the system. More specifically, if $(a, b, c)$ are the parameters from (1.2) of the nematic liquid crystal used in the homogenisation process and $\left(a^{\prime}, b^{\prime}, c^{\prime}\right)$ are the desired parameters for the homogenised material, our goal is to choose the lenghts of the model particle used for constructing the scaffold and a surface energy density $f_{s}$ such that if, for example, the bulk energy chosen is the one from (1.2), then, in the limit $\varepsilon \rightarrow 0$, we want to obtain a $f_{\text {hom }}$ with the following property:

$$
f_{b}(Q)+f_{\text {hom }}(Q)=a^{\prime} \operatorname{tr}\left(Q^{2}\right)-b^{\prime} \operatorname{tr}\left(Q^{3}\right)+c^{\prime} \operatorname{tr}\left(Q^{2}\right)^{2} .
$$

The article is organised in the following manner:

- in Section 2 we present the technical assumptions of the problem;

- in Section 3 we present the main results of this work: a general result together with its applications to the Landau-de Gennes model;

- in Section 4 we present the study of the properties of the functional $\mathcal{F}_{\varepsilon}$ for a fixed value of $\varepsilon>0$;

- in Section 5 we glue together the properties studied in the previous section and analyse the $\Gamma$-limit of $\mathcal{F}_{\varepsilon}$ as $\varepsilon \rightarrow 0$ and we prove the main theorems stated in Section 3;

- in Section 6 we analyse the rate of convergence of the sequence of surface energies to the homogenised surface functional, where the main result is Proposition 6.4, but we also analyse the rate of convergence of the sequence of minimisers of the free energies to a minimiser of the homogenised free energy (see Rem. 6.5)

and

- in Appendix A we prove various results, the most important of which is the proposition regarding the explicit extension function that we use in Section 4.1.

\section{Notations And technical assumptions}

Let $\Omega \subset \mathbb{R}^{3}$ be a bounded, Lipschitz domain, that models the ambient liquid crystal, and let $\mathcal{C} \subset \mathbb{R}^{3}$ be the model particle for the cubic microlattice. Since $\Omega$ is bounded in $\mathbb{R}^{3}$, then:

$$
\exists L_{0}, l_{0}, h_{0} \in[0,+\infty) \text { such that } \bar{\Omega} \subseteq\left[-L_{0}, L_{0}\right] \times\left[-l_{0}, l_{0}\right] \times\left[-h_{0}, h_{0}\right] .
$$

In Figure 2, we illustrate some examples of cubic microlattices, where the "connecting" boxes (which can be seen better in Figure 1 as being the black cubes) are cubes of size $\varepsilon^{\alpha}$, with $\alpha=1.4999^{2}$ and $\varepsilon$ has a positive value close to 0 , since we desire to work in the dilute regime. The distance between two closest black cubes is

\footnotetext{
${ }^{2}$ We choose $\alpha$ close to the value $3 / 2$ in order to make the difference between the lengths of the sides of the black cubes and the gray parallelepipeds from Figure 1 more visible, for relatively "large" values of $\varepsilon(0.01,0.05$ or 0.001$)$.
} 


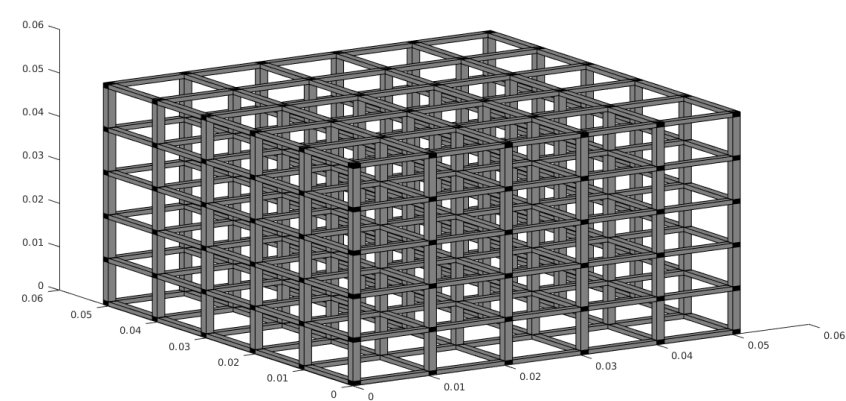

(a) $\varepsilon=0.01$ and $l=0.05$;

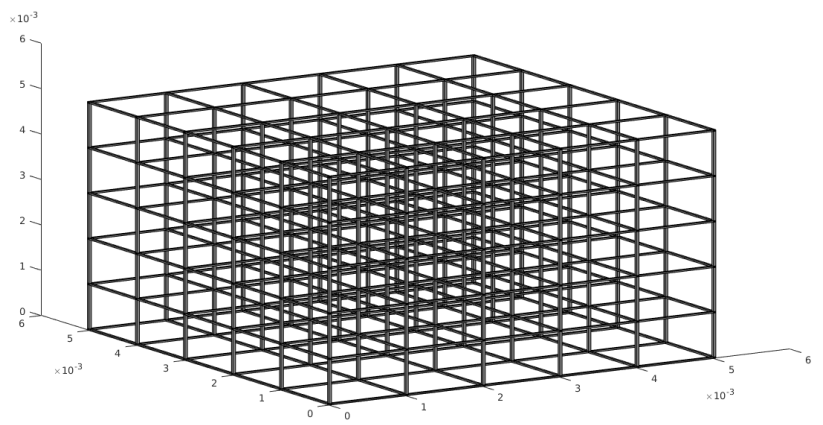

(b) $\varepsilon=0.001$ and $l=0.005$.

Figure 2. Cubic microlattices constructed in the box $[0, l]^{3}$ with $\alpha=1.4999$.

equal to $\varepsilon$, therefore the length of the black cubes is significantly smaller than the distance between them, by using the exponent $\alpha .{ }^{3}$ For Figure 1 , we used $\varepsilon=0.05, \alpha=1.4999$ and $l=0.25$, so we keep the same ratio between $\varepsilon$ and $l$ as in Figure 2.

In order to construct such a scaffold, we use as a model particle the cube:

$$
\mathcal{C}=\left[-\frac{1}{2}, \frac{1}{2}\right]^{3}
$$

We denote by $\partial \mathcal{C}$ the surface of the cube $\mathcal{C}$, which we also write it as:

$$
\partial \mathcal{C}=\mathcal{C}^{x} \cup \mathcal{C}^{y} \cup \mathcal{C}^{z}
$$

where $\mathcal{C}^{x}$ is the union of the two faces of the cube that are perpendicular to the $x$ direction and in the same way are defined $\mathcal{C}^{y}$ and $\mathcal{C}^{z}$.

Then, for a fixed value of $\varepsilon>0$ and an $\varepsilon$-independent positive constant $\alpha$, we define

$$
\mathcal{C}^{\alpha}=\left[-\frac{\varepsilon^{\alpha}}{2 p},+\frac{\varepsilon^{\alpha}}{2 p}\right] \times\left[-\frac{\varepsilon^{\alpha}}{2 q},+\frac{\varepsilon^{\alpha}}{2 q}\right] \times\left[-\frac{\varepsilon^{\alpha}}{2 r},+\frac{\varepsilon^{\alpha}}{2 r}\right],
$$

with $p, q, r \in[1,+\infty)$.

Remark 2.1. Using the notion of cubic symmetry described in introduction, we call the scaffold symmetric whenever $p=q=r$. In Figures 1 and 2, we illustrate only the symmetric case $p=q=r=1$.

We construct now the lattice

$$
\mathcal{X}_{\varepsilon}=\left\{x \in \Omega: x=\left(x_{1}, x_{2}, x_{3}\right), \operatorname{dist}(x, \partial \Omega) \geq \varepsilon \text { and } x_{k} / \varepsilon \in \mathbb{Z} \text { for } k \in \overline{1,3}\right\},
$$

which we rewrite it as:

$$
\mathcal{X}_{\varepsilon}=\left\{x_{\varepsilon}^{i}: i \in \overline{1, N_{\varepsilon}}\right\} \text {, where } N_{\varepsilon}=\operatorname{card}\left(\mathcal{X}_{\varepsilon}\right) .
$$

\footnotetext{
${ }^{3}$ The reason why we represent the lattice only in the box $[0, l]^{3}$, with $l=5 \varepsilon$, is that if we keep the same $l$ and shrink $\varepsilon$, then the number of boxes appearing in the image would be significantly larger, hence, as we make $\varepsilon$ smaller, we also zoom in to have a better picture of what is happening for small values of $\varepsilon$.
} 
Hence, the first part of the scaffold is the family of parallelepipeds

$$
\mathcal{C}_{\varepsilon}=\bigcup_{i=1}^{N_{\varepsilon}} \mathcal{C}_{\varepsilon}^{i} \text {, where } \mathcal{C}_{\varepsilon}^{i}=x_{\varepsilon}^{i}+\mathcal{C}^{\alpha}, \text { for every } i \in \overline{1, N_{\varepsilon}},
$$

which represents the union of all black parallelepipeds from Figure 1.

Remark 2.2. We call throughout this work the black parallelepipeds constructed in (2.7) the "inner particles" of the scaffold or the "inner parallelepipeds" or the "inner boxes". In this way, $\mathcal{C}_{\varepsilon}$ represents the set of all "inner parallelepipeds". We choose the term "inner" because most of these particles will interact with the nematic liquid crystal material only on their edges and most of them are not visible, as shown in Figure 1, except for those which will be close to the boundary of the domain $\Omega$.

We add now the lattice

$$
\begin{aligned}
\mathcal{Y}_{\varepsilon} & :=\left\{y_{\varepsilon} \in \Omega: \exists i, j \in \overline{1, N_{\varepsilon}} \text { such that }\left|x_{\varepsilon}^{i}-x_{\varepsilon}^{j}\right|=\varepsilon \text { and } y_{\varepsilon}=\frac{1}{2}\left(x_{\varepsilon}^{i}+x_{\varepsilon}^{j}\right)\right\} \\
\text { and let } M_{\varepsilon} & =\operatorname{card}\left(\mathcal{Y}_{\varepsilon}\right) .
\end{aligned}
$$

In each of the points from the lattice $\mathcal{Y}_{\varepsilon}$ we construct a gray parallelepiped, as shown in Figure 1.

Remark 2.3. We call throughout this work the gray parallelepipeds from Figure 1 the "connecting parallelepipeds" of the scaffold or the "connecting particles" or the "connecting boxes". The reason why we use this notation is because any single "connecting particle" joins two different "inner particles", for which their centers are at $\varepsilon$ distance apart from each other.

Remark 2.4. We can interpret now more easily the "inner parallelepipeds" which are close to the $\partial \Omega$ by observing that it has less than 6 adjacent "connecting parallelepipeds". If it has 6 , then that "inner parallelepiped" will not be "visible" (in Fig. 1) and further away from $\partial \Omega$. Moreover, we prove in Section 4.2 that all the "inner parallelepipeds" have no contribution to the limiting problem, regardless whether they are close to the boundary of $\Omega$ or not. This is mainly because the "inner parallelepipeds" which have 6 adjacent "connecting parallelepipeds" touch the nematic liquid crystal only on their edges (which are of measure zero) and the "inner parallelepipeds" which have less than 6 , their contribution becomes negligible due to their "small" number. More details can be found in Section 4.2.

We split this lattice into three parts, since the "connecting parallelepipeds" are elongated into three different directions, granted by the axes of the Cartesian coordinate system in $\mathbb{R}^{3}$. We denote by $\mathcal{P}_{\varepsilon}$ the union of all of "connecting parallelepipeds".

Since the scaffold is the union between the "inner particles" and the "connecting particles", we denote the scaffold as $\mathcal{N}_{\varepsilon}=\mathcal{C}_{\varepsilon} \cup \mathcal{P}_{\varepsilon}$ with $\partial \mathcal{N}_{\varepsilon}$ its surface.

More details regarding the construction of these objects can be found in Appendix A.1.

Remark 2.5. In this paper, we use the notation $A \lesssim B$ for two real numbers $A$ and $B$ whenever there exists an $\varepsilon$-independent constant $C$ such that $A \leq C \cdot B$.

We assume furthermore that:

$\left(A_{1}\right) \Omega \subset \mathbb{R}^{3}$ is a smooth and bounded domain

$\left(A_{2}\right) 1<\alpha<2$;

Remark 2.6. The condition $1<\alpha$ ensures that the "connecting particles" exist and also the dilute regime of the homogenisation problem. This is explained more in Appendix A.1 and in Remark A.1. The reason why we impose other bound comes from the fact that if $\alpha>2$, then equicoercivity may be lost. An example of this 
situation is described in Lemma 4.8 and we follow the same directions as in Lemma 3.6 from [13]. Another way of understanding why we impose this upper bound is given by the factor in front of the surface energy: $\frac{\varepsilon^{3}}{\varepsilon^{\alpha}\left(\varepsilon-\varepsilon^{\alpha}\right)}$, which can be seen as $\frac{\varepsilon^{2-\alpha}}{1-\varepsilon^{\alpha-1}}$. Since $\alpha>1,1-\varepsilon^{\alpha-1} \rightarrow 1$ as $\varepsilon \rightarrow 0$, hence the factor in front of the surface energy behaves like $\varepsilon^{2-\alpha}$. As a comparison, in [13], the factor in front of the surface energy is $\varepsilon^{3-2 \alpha}$ and the upper bound used there is $\alpha<3 / 2$.

$\left(A_{3}\right)$ There exists a constant $\lambda_{\Omega}>0$ such that

$$
\operatorname{dist}\left(z_{\varepsilon}^{i}, \partial \Omega\right)+\frac{1}{2} \inf _{j \neq i}\left|z_{\varepsilon}^{j}-z_{\varepsilon}^{i}\right| \geq \lambda_{\Omega} \varepsilon
$$

for any $\varepsilon>0$ and any center $z_{\varepsilon}^{i}$ of an object (either a "inner" or "connecting" parallelepiped) that is contained within the cubic microlattice, where $i \in \overline{1,\left(N_{\varepsilon}+M_{\varepsilon}\right)}$.

$\left(A_{4}\right)$ As $\varepsilon \rightarrow 0$, the measures

$$
\mu_{\varepsilon}^{X}:=\varepsilon^{3} \sum_{k=1}^{X_{\varepsilon}} \delta_{y_{\varepsilon}^{x, k}}, \mu_{\varepsilon}^{Y}:=\varepsilon^{3} \sum_{l=1}^{Y_{\varepsilon}} \delta_{y_{\varepsilon}^{y, l}} \text { and } \mu_{\varepsilon}^{Z}:=\varepsilon^{3} \sum_{m=1}^{Z_{\varepsilon}} \delta_{y_{\varepsilon}^{z, m}}
$$

converge weakly* (as measures in $\mathbb{R}^{3}$ ) to the Lebesgue measure restricted on $\Omega$, denoted $\mathrm{dx} L \Omega$.

We say that a function $f: \mathcal{S}_{0} \otimes \mathbb{R}^{3} \rightarrow \mathbb{R}$ is strongly convex if there exists $\theta>0$ such that the function $\tilde{f}: \mathcal{S}_{0} \otimes \mathbb{R}^{3} \rightarrow \mathbb{R}$ defined by $\tilde{f}(D)=f(D)-\theta|D|^{2}$ is convex.

$\left(A_{5}\right) f_{e}: \mathcal{S}_{0} \otimes \mathbb{R}^{3} \rightarrow[0,+\infty)$ is differentiable, strongly convex and there exists a constant $\lambda_{e}>0$ such that

$$
\lambda_{e}^{-1}|D|^{2} \leq f_{e}(D) \leq \lambda_{e}|D|^{2}, \quad\left|\left(\nabla f_{e}\right)(D)\right| \leq \lambda_{e}(|D|+1)
$$

for any $D \in \mathcal{S}_{0} \times \mathbb{R}^{3}$.

$\left(A_{6}\right) f_{b}: \mathcal{S}_{0} \rightarrow \mathbb{R}$ is continuous, bounded from below and there exists a constant $\lambda_{b}>0$ such that $\left|f_{b}(Q)\right| \leq \lambda_{b}\left(|Q|^{6}+1\right)$ for any $Q \in \mathcal{S}_{0}$.

$\left(A_{7}\right) f_{s}: \mathcal{S}_{0} \times \mathbb{S}^{2} \rightarrow \mathbb{R}$ is continuous and there exists a strictly positive constant $\lambda_{s}$ such that, for any $Q_{1}, Q_{2} \in \mathcal{S}_{0}$ and any $\nu \in \mathbb{S}^{2}$, we have

$$
\left|f_{s}\left(Q_{1}, \nu\right)-f_{s}\left(Q_{2}, \nu\right)\right| \leq \lambda_{s}\left|Q_{1}-Q_{2}\right|\left(\left|Q_{1}\right|^{3}+\left|Q_{2}\right|^{3}+1\right)
$$

It is easy to see from here that $f_{s}$ has a quartic growth in $Q$.

\section{MAin Results}

\subsection{General case}

Let $f_{\text {hom }}: \mathcal{S}_{0} \rightarrow \mathbb{R}$ be the function defined as:

$$
f_{h o m}(Q):=\frac{q+r}{q r} \int_{\mathcal{C}^{x}} f_{s}(Q, \nu) \mathrm{d} \sigma+\frac{p+r}{p r} \int_{\mathcal{C}^{y}} f_{s}(Q, \nu) \mathrm{d} \sigma+\frac{p+q}{p q} \int_{\mathcal{C}^{z}} f_{s}(Q, \nu) \mathrm{d} \sigma
$$

for any $Q \in \mathcal{S}_{0}$, where $\mathcal{C}^{x}, \mathcal{C}^{y}$ and $\mathcal{C}^{z}$ are defined in (2.3). From $\left(A_{7}\right)$, we can deduce that $f_{\text {hom }}$ is also continuous and that it has a quartic growth. If we work in the symmetric case, that is $p=q=r$, then relation (3.1) becomes: 


$$
f_{h o m}(Q):=\frac{2}{p} \int_{\partial \mathcal{C}} f_{s}(Q, \nu) \mathrm{d} \sigma
$$

Remark 3.1. Throughout this paper, the function $f_{\text {hom }}$ is sometimes referred to as being the homogenised functional, simply because it represents the effect that arises from the surface energy term in the limiting free energy functional.

The main results of these notes concerns the asymptotic behaviour of local minimisers of the functional $\mathcal{F}_{\varepsilon}$, as $\varepsilon \rightarrow 0$.

Let $g \in H^{1 / 2}\left(\partial \Omega, \mathcal{S}_{0}\right)$ be a boundary datum. We denote by $H_{g}^{1}\left(\Omega, \mathcal{S}_{0}\right)$ the set of maps $Q$ from $H^{1}\left(\Omega, \mathcal{S}_{0}\right)$ such that $Q=g$ on $\partial \Omega$ in the trace sense. Similarly, we define $H_{g}^{1}\left(\Omega_{\varepsilon}, \mathcal{S}_{0}\right)$ to be $H^{1}\left(\Omega_{\varepsilon}\right)$ with $Q=g$ on $\partial \Omega$ in the trace sense.

We use the harmonic extension operator, $E_{\varepsilon}: H_{g}^{1}\left(\Omega_{\varepsilon}, \mathcal{S}_{0}\right) \rightarrow H_{g}^{1}\left(\Omega, \mathcal{S}_{0}\right)$, defined in the following way: $E_{\varepsilon} Q:=$ $Q$ on $\Omega_{\varepsilon}$ and inside the scaffold, $E_{\varepsilon} Q$ is the unique solution of the following problem:

$$
\begin{cases}\Delta E_{\varepsilon} Q=0 & \text { in } \mathcal{N}_{\varepsilon} \\ E_{\varepsilon} Q \equiv Q & \text { on } \partial \mathcal{N}_{\varepsilon}\end{cases}
$$

Using this framework, we can produce the main result of this work:

Theorem 3.2. Suppose that the assumptions $\left(A_{1}\right)-\left(A_{7}\right)$ are satisfied. Let $Q_{0} \in H_{g}^{1}\left(\Omega, \mathcal{S}_{0}\right)$ be an isolated $H^{1}$ local minimiser for $\mathcal{F}_{0}$, defined in (1.3), that is, there exists $\delta_{0}>0$ such that $\mathcal{F}_{0}\left[Q_{0}\right]<\mathcal{F}_{0}[Q]$ for any $Q \in$ $H_{g}^{1}\left(\Omega, \mathcal{S}_{0}\right)$ such that $\left\|Q-Q_{0}\right\|_{H_{g}^{1}\left(\Omega, \mathcal{S}_{0}\right)} \leq \delta_{0}$ and $Q \neq Q_{0}$. Then for any $\varepsilon$ sufficiently small enough, there exists a sequence of $H^{1}$-local minimisers $Q_{\varepsilon}$ of $\mathcal{F}_{\varepsilon}$ such that $E_{\varepsilon} Q_{\varepsilon} \rightarrow Q_{0}$ strongly in $H_{g}^{1}\left(\Omega, \mathcal{S}_{0}\right)$.

\subsection{Applications to the Landau-de Gennes model}

In this subsection, we particularise Theorem 3.2 to the case of the Landau-de Gennes model. Before doing this, let us introduce first some of the energies used in this model for nematic liquid crystals.

\section{- The elastic energy}

We consider the following form for the elastic energy:

$$
f_{e}(\nabla Q):=\sum_{i, j, k \in\{1,2,3\}}\left[\frac{L_{1}}{2}\left(\frac{\partial Q_{i j}}{\partial x_{k}}\right)^{2}+\frac{L_{2}}{2} \frac{\partial Q_{i j}}{\partial x_{j}} \frac{\partial Q_{i k}}{\partial x_{k}}+\frac{L_{3}}{2} \frac{\partial Q_{i k}}{\partial x_{j}} \frac{\partial Q_{i j}}{\partial x_{k}}\right],
$$

where $Q_{i j}$ is the $(i j)^{t h}$ component of $Q,\left(x_{1}, x_{2}, x_{3}\right)$ represents the usual cartesian coordinates and $e_{i j k}$ represents the Levi-Civita symbol.

In order to fulfill assumption $\left(A_{5}\right)$, we take as in [19]:

$$
L_{1}>0, \quad-L_{1}<L_{3}<2 L_{1}, \quad-\frac{3}{5} L_{1}-\frac{1}{10} L_{3}<L_{2} .
$$

\section{- The bulk energy}

For the bulk energy density, we use several versions of it. The first one is the classical quartic polynomial in the scalar invariants of $Q$, defined in (1.2), which verifies the conditions of assumption $\left(A_{4}\right)$ :

$$
f_{b}(Q)=a \operatorname{tr}\left(Q^{2}\right)-b \operatorname{tr}\left(Q^{3}\right)+c \operatorname{tr}\left(Q^{2}\right)^{2} .
$$


We also prove similar results for a general polynomial in the scalar invariants of $Q$, that is:

$$
f_{b}^{g e n}(Q)=\sum_{k=2}^{N} a_{k} \operatorname{tr}\left(Q^{k}\right),
$$

where $N \in \mathbb{N}, N \geq 4$ is fixed, with the coefficients $a_{k} \in \mathbb{R}$ chosen such that the polynomial $h: \mathbb{R} \rightarrow \mathbb{R}$, defined by $h(x)=\sum_{k=2}^{N} a_{k} x^{k}$, for any $x \in \mathbb{R}$, admits at least one local minimum over $\mathbb{R}$.

- The surface energy

For each of the versions of the bulk energy densities, we choose suitable surface energy densities, such that, in the homogenised functional, the surface terms grant an effect $f_{h o m}$ which has the same form as the initial bulk energy chosen, but with different coefficients, most important of which the coefficient of $\operatorname{tr}\left(Q^{2}\right)$ is now different. Therefore, our choice of the surface energy density has a strong connection with the bulk energy density chosen.

Moreover, since Theorem 3.2 holds for any values of $p, q$ and $r$, that is, for any type of parallelepiped chosen for the construction of the scaffold, and since, in reality, 2PP (two-photon polymerization) materials with cubic symmetry properties, in the sense from Remark 2.1, have been obtained (for example, in [23]), then we also split our work on whether the scaffold is symmetric or not.

Hence, our choices of surface energy densities will depend on the bulk energy density chosen and if the scaffold is symmetric or not.

I) If the scaffold is symmetric, as described in Remark 2.1, then the physical invariances require

$$
f_{s}\left(U Q U^{T}, U u\right)=f_{s}(Q, u), \forall(Q, u) \in \mathcal{S}_{0} \times \mathbb{R}^{3}, U \in \mathcal{O}(3)
$$

and this leads, according to Proposition 2.6 from [13], to a surface energy of the form

$$
f_{s}(Q, \nu)=\tilde{f}_{s}\left(\operatorname{tr}\left(Q^{2}\right), \operatorname{tr}\left(Q^{3}\right), \nu \cdot Q \nu, \nu \cdot Q^{2} \nu\right), \forall(Q, \nu) \in \mathcal{S}_{0} \times \mathbb{R}^{3} .
$$

1) Let us consider the case in which the bulk energy is the classical Landau-de Gennes quartic polynomial in $Q$, described by (1.2). In this case, we use one of the most common forms for the surface energy, which is the Rapini-Papoular energy:

$$
f_{s}(Q, \nu)=W \operatorname{tr}\left(Q-s_{+}\left(\nu \otimes \nu-\mathbb{I}_{3} / 3\right)\right)^{2},
$$

where $W$ is a coefficient measuring the strength of the anchoring, $s_{+}$is measuring the deviation from the homeotropic (perpendicular) anchoring to the boundary and $\mathbb{I}_{3}$ is the $3 \times 3$ identity matrix.

Another surface energy density that we use, which verifies (3.5), is the following:

$$
f_{s}(Q, \nu)=k_{a}\left(\nu \cdot Q^{2} \nu\right)+k_{b}(\nu \cdot Q \nu)\left(\nu \cdot Q^{2} \nu\right)+k_{c}\left(\nu \cdot Q^{2} \nu\right)^{2}+a^{\prime} \operatorname{tr}\left(Q^{2}\right)+\frac{2 b^{\prime}}{3} \operatorname{tr}\left(Q^{3}\right)+\frac{c^{\prime}}{2} \operatorname{tr}\left(Q^{2}\right)^{2},
$$

where $k_{a}, k_{b}, k_{c}, a^{\prime}, b^{\prime}$ and $c^{\prime}$ are constants.

Remark 3.3. If our choice of $f_{s}$ contains terms of the form $\operatorname{tr}\left(Q^{2}\right)$ or $\operatorname{tr}\left(Q^{3}\right)$, then these terms very easily generate in $f_{\text {hom }}$ terms similar with the one from the bulk energy defined in (1.2), since they are exactly the same. Our goal in the paragraphs is to use the other terms from (3.7) for the surface energy densities, which are of the form $\nu \cdot Q^{k} \nu$ and for which the previous implication is not that immediate.

2) For the bulk energy density in (3.4), we choose a more general form for $f_{s}(Q, \nu)$, depending only on terms of the form $\nu \cdot Q^{k} \nu$. In this situation, the function $f_{\text {hom }}$ can be computed easily, due to the geometry of the scaffold. More specifically, according to Proposition A.8, we obtain in the homogenised functional terms of the 
form $\operatorname{tr}\left(Q^{k}\right)$, with $k \geq 4$, but they only depend on $\operatorname{tr}\left(Q^{2}\right)$ and $\operatorname{tr}\left(Q^{3}\right)$, since $\operatorname{tr}(Q)=0$. In order to prove this statement, let $\lambda_{1}, \lambda_{2}$ and $\lambda_{3}$ the eigenvalues of $Q$. Then they satisfy the system:

$$
\left\{\begin{array}{l}
\lambda_{1}+\lambda_{2}+\lambda_{3}=0 \\
\lambda_{1}^{2}+\lambda_{2}^{2}+\lambda_{3}^{2}=\operatorname{tr}\left(Q^{2}\right) \\
\lambda_{1}^{3}+\lambda_{2}^{3}+\lambda_{3}^{3}=\operatorname{tr}\left(Q^{3}\right)
\end{array}\right.
$$

and, by solving the system, we can see that $\lambda_{1}, \lambda_{2}$ and $\lambda_{3}$ can be viewed as functions of $\operatorname{tr}\left(Q^{2}\right)$ and $\operatorname{tr}\left(Q^{3}\right)$. Since $\operatorname{tr}\left(Q^{k}\right)=\lambda_{1}^{k}+\lambda_{2}^{k}+\lambda_{3}^{k}$, for any $k \in \mathbb{N}, k \geq 1$, then it is easy to see from here that $\operatorname{tr}\left(Q^{k}\right)$, for $k \geq 4$, is depending only on $\operatorname{tr}\left(Q^{2}\right)$ and $\operatorname{tr}\left(Q^{3}\right)$. Indeed, by Cayley-Hamilton theorem, the identity:

$$
Q^{3}-\frac{1}{2} \operatorname{tr}\left(Q^{2}\right) Q-\frac{1}{3} \operatorname{tr}\left(Q^{3}\right) \mathbb{I}_{3}=0
$$

becomes valid for any Q-tensor $Q$, where $\mathbb{I}_{3}$ is the $3 \times 3$ identity matrix. Multiplying this identity succcessively by $Q, Q^{2}, Q^{3}$ and so on and taking the trace we obtain the claim.

II) If the scaffold does not present cubic symmetry, in the sense of Remark 2.1, we only illustrate the case in which the bulk energy density is the one from (1.2) and the surface energy density is a variation of (3.7).

The goal of the next subsubsections is to analyse each particular case described above and to obtain similar results as Theorem 3.2 for each of it.

\subsubsection{The symmetric case: $p=q=r$}

Assuming $p=q=r$ implies that the "inner" parallelepipeds constructed in (2.7) are actually cubes.

1. We analyse first the case in which $f_{b}$ is defined in (1.2), that is:

$$
f_{b}(Q)=a \operatorname{tr}\left(Q^{2}\right)-b \operatorname{tr}\left(Q^{3}\right)+c \operatorname{tr}\left(Q^{2}\right)^{2}
$$

a) We analyse the case when $(a, b, c) \rightsquigarrow\left(a^{\prime}, b^{\prime} c^{\prime}\right)$, where all the parameters are non-zero and $c$ and $c^{\prime}$ are positive, which by " $\rightsquigarrow$ " we mean that from a nematic liquid crystal with the parameters $(a, b, c)$ we want to generate a new homogenised material, which also behaves like a NLC, but with parameters $\left(a^{\prime}, b^{\prime}, c^{\prime}\right)$.

We choose $f_{s}$ in this case to be:

$$
f_{s}^{L D G}(Q, \nu)=\frac{p}{4}\left(\left(a^{\prime}-a\right)\left(\nu \cdot Q^{2} \nu\right)-\left(b^{\prime}-b\right)\left(\nu \cdot Q^{3} \nu\right)+2\left(c^{\prime}-c\right)\left(\nu \cdot Q^{4} \nu\right)\right)
$$

where $a^{\prime}, b^{\prime}$ and $c^{\prime}$ are the desired coefficients in the homogenised bulk potential, such that in the homogenised material, we have:

$$
f_{\text {hom }}^{L D G}(Q)=\left(a^{\prime}-a\right) \operatorname{tr}\left(Q^{2}\right)-\left(b^{\prime}-b\right) \operatorname{tr}\left(Q^{3}\right)+\left(c^{\prime}-c\right)\left(\operatorname{tr}\left(Q^{2}\right)\right)^{2} .
$$

We are interested in studying the behaviour of the whole material when $\varepsilon \rightarrow 0$, that is, studying the following functionals:

$$
\mathcal{F}_{\varepsilon}^{L D G}\left[Q_{\varepsilon}\right]:=\int_{\Omega_{\varepsilon}}\left(f_{e}\left(\nabla Q_{\varepsilon}\right)+a \operatorname{tr}\left(Q_{\varepsilon}^{2}\right)-b \operatorname{tr}\left(Q_{\varepsilon}^{3}\right)+c\left(\operatorname{tr}\left(Q_{\varepsilon}^{2}\right)\right)^{2}\right) \mathrm{d} x+\frac{\varepsilon^{3-\alpha}}{\varepsilon-\varepsilon^{\alpha}} \int_{\partial \mathcal{N}_{\varepsilon}} f_{s}^{L D G}\left(Q_{\varepsilon}, \nu\right) \mathrm{d} \sigma
$$


and

$$
\mathcal{F}_{0}^{L D G}[Q]:=\int_{\Omega}\left(f_{e}(\nabla Q)+a^{\prime} \operatorname{tr}\left(Q^{2}\right)-b^{\prime} \operatorname{tr}\left(Q^{3}\right)+c^{\prime}\left(\operatorname{tr}\left(Q^{2}\right)\right)^{2}\right) \mathrm{d} x
$$

Theorem 3.4. Let $(a, b, c)$ and $\left(a^{\prime}, b^{\prime}, c^{\prime}\right)$ be two set of parameters with $c>0$ and $c^{\prime}>0$. Suppose that the assumptions $\left(A_{1}\right)-\left(A_{7}\right)$ are satisfied and also the inequalities from (3.3). Then, for any isolated $H^{1}$-local minimiser $Q_{0}$ of the functional $\mathcal{F}_{0}^{L D G}$ defined by (3.11), and for $\varepsilon>0$ sufficiently small enough, there exists a sequence of local minimisers $Q_{\varepsilon}$ of the functionals $\mathcal{F}_{\varepsilon}^{L D G}$, defined by (3.10), such that $E_{\varepsilon} Q_{\varepsilon} \rightarrow Q_{0}$ strongly in $H_{g}^{1}\left(\Omega, \mathcal{S}_{0}\right)$.

Proof. This theorem is a particular case of Theorem 3.2. It is sufficient to prove that relation (3.9) can be obtained via (3.2), that is:

$$
f_{h o m}^{L D G}(Q)=\frac{2}{p} \int_{\partial \mathcal{C}} f_{s}^{L D G}(Q, \nu) \mathrm{d} \sigma=\left(a^{\prime}-a\right) \operatorname{tr}\left(Q^{2}\right)-\left(b^{\prime}-b\right) \operatorname{tr}\left(Q^{3}\right)+\left(c^{\prime}-c\right)\left(\operatorname{tr}\left(Q^{2}\right)\right)^{2} .
$$

Using Proposition A.8, we have:

$$
\int_{\partial \mathcal{C}} \nu \cdot Q^{2} \nu \mathrm{d} \sigma=2 \operatorname{tr}\left(Q^{2}\right), \quad \int_{\partial \mathcal{C}} \nu \cdot Q^{3} \nu \mathrm{d} \sigma=2 \operatorname{tr}\left(Q^{3}\right) \quad \text { and } \quad \int_{\partial \mathcal{C}} \nu \cdot Q^{4} \nu \mathrm{d} \sigma=2 \operatorname{tr}\left(Q^{4}\right),
$$

from which we get

$$
\begin{aligned}
\frac{2}{p} \int_{\partial \mathcal{C}} f_{s}^{L D G}(Q, \nu) \mathrm{d} \sigma & =\frac{2}{p} \cdot \frac{p}{4}\left(\left(a^{\prime}-a\right) \cdot 2 \operatorname{tr}\left(Q^{2}\right)-\left(b^{\prime}-b\right) \cdot 2 \operatorname{tr}\left(Q^{3}\right)+2\left(c^{\prime}-c\right) \cdot 2 \operatorname{tr}\left(Q^{4}\right)\right) \\
\Rightarrow f_{h o m}^{L D G}(Q) & =\left(a^{\prime}-a\right) \operatorname{tr}\left(Q^{2}\right)-\left(b^{\prime}-b\right) \operatorname{tr}\left(Q^{3}\right)+\left(c^{\prime}-c\right) \cdot 2 \operatorname{tr}\left(Q^{4}\right) .
\end{aligned}
$$

Since $Q \in \mathcal{S}_{0}$, then, by Cayley-Hamilton theorem, if $\lambda_{1}, \lambda_{2}$ and $\lambda_{3}$ are the eigenvalues of $Q$, we have:

$$
\left\{\begin{array}{l}
\lambda_{1}+\lambda_{2}+\lambda_{3}=\operatorname{tr}(Q)=0 \\
\lambda_{1} \lambda_{2}+\lambda_{2} \lambda_{3}+\lambda_{3} \lambda_{1}=\frac{1}{2}\left((\operatorname{tr}(Q))^{2}-\operatorname{tr}\left(Q^{2}\right)\right)=-\frac{1}{2} \operatorname{tr}\left(Q^{2}\right)
\end{array}\right.
$$

and

$$
\begin{aligned}
\operatorname{tr}\left(Q^{4}\right)=\lambda_{1}^{4}+\lambda_{2}^{4}+\lambda_{3}^{4} & =\left(\lambda_{1}^{2}+\lambda_{2}^{2}+\lambda_{3}^{2}\right)^{2}-2\left(\lambda_{1}^{2} \lambda_{2}^{2}+\lambda_{2}^{2} \lambda_{3}^{2}+\lambda_{3}^{2} \lambda_{1}^{2}\right) \\
& =\left(\lambda_{1}^{2}+\lambda_{2}^{2}+\lambda_{3}^{2}\right)^{2}-2\left(\left(\lambda_{1} \lambda_{2}+\lambda_{2} \lambda_{3}+\lambda_{3} \lambda_{1}\right)^{2}-2 \lambda_{1} \lambda_{2} \lambda_{3}\left(\lambda_{1}+\lambda_{2}+\lambda_{3}\right)\right) \\
& =\left(\operatorname{tr}\left(Q^{2}\right)\right)^{2}-2\left(-\frac{1}{2} \operatorname{tr}\left(Q^{2}\right)\right)^{2} \\
& =\frac{1}{2}\left(\operatorname{tr}\left(Q^{2}\right)\right)^{2}
\end{aligned}
$$

from which we get the relation $2 \operatorname{tr}\left(Q^{4}\right)=\left(\operatorname{tr}\left(Q^{2}\right)\right)^{2}$.

Hence, we conclude that:

$$
f_{\text {hom }}^{L D G}(Q)=\left(a^{\prime}-a\right) \operatorname{tr}\left(Q^{2}\right)-\left(b^{\prime}-b\right) \operatorname{tr}\left(Q^{3}\right)+\left(c^{\prime}-c\right)\left(\operatorname{tr}\left(Q^{2}\right)\right)^{2} .
$$


b) We analyse now the case in which we want $(a, 0,0) \rightsquigarrow\left(a^{\prime}, 0,0\right)$, with $a$ and $a^{\prime}$ non-zero. In this situation, we have

$$
f_{b}^{R P}(Q)=a \operatorname{tr}\left(Q^{2}\right)
$$

and we choose $f_{s}$ to be given by the Rapini-Papoular form (3.6):

$$
f_{s}^{R P}(Q, \nu)=\frac{p}{12}\left(a^{\prime}-a\right) \operatorname{tr}\left(Q-Q_{\nu}\right)^{2}
$$

where $Q_{\nu}=\nu \otimes \nu-\mathbb{I}_{3} / 3$ and $\mathbb{I}_{3}$ is the $3 \times 3$ identity matrix.

In this case, we have:

$$
\mathcal{F}_{\varepsilon}^{R P}\left[Q_{\varepsilon}\right]:=\int_{\Omega_{\varepsilon}}\left(f_{e}\left(\nabla Q_{\varepsilon}\right)+a \operatorname{tr}\left(Q_{\varepsilon}^{2}\right)\right) \mathrm{d} x+\frac{p}{2} \cdot\left(a^{\prime}-a\right) \cdot\left(\frac{\varepsilon^{3-\alpha}}{\varepsilon-\varepsilon^{\alpha}} \int_{\partial \mathcal{N}_{\varepsilon}} \operatorname{tr}\left(Q_{\varepsilon}-Q_{\nu}\right)^{2} \mathrm{~d} \sigma\right)
$$

and we prove that

$$
f_{\text {hom }}^{R P}(Q)=\left(a^{\prime}-a\right) \operatorname{tr}\left(Q^{2}\right)
$$

and

$$
\mathcal{F}_{0}^{R P}[Q]:=\int_{\Omega}\left(f_{e}(\nabla Q)+a^{\prime} \operatorname{tr}\left(Q^{2}\right)\right) \mathrm{d} x
$$

Theorem 3.5. Let $a$ and $a^{\prime}$ be two parameters. Suppose that the assumptions $\left(A_{1}\right)-\left(A_{7}\right)$ are satisfied and also the inequalities from (3.3). Then, for any isolated $H^{1}$-local minimiser $Q_{0}$ of the functional $\mathcal{F}_{0}^{R P}$ defined by (3.15), and for $\varepsilon>0$ sufficiently small enough, there exists a sequence of local minimisers $Q_{\varepsilon}$ of the functionals $\mathcal{F}_{\varepsilon}^{R P}$, defined by $(3.13)$, such that $E_{\varepsilon} Q_{\varepsilon} \rightarrow Q_{0}$ strongly in $H_{g}^{1}\left(\Omega, \mathcal{S}_{0}\right)$.

Proof. The proof follows the same steps as in the proof of Theorem 3.4, using Proposition A.9. We only have to prove that relation (3.14) can be obtained using (3.2), knowing that (3.12) holds.

From (3.2) and Proposition A.9, we have:

$$
\begin{aligned}
f_{h o m}^{R P}(Q) & =\frac{2}{p} \int_{\partial \mathcal{C}} f_{s}^{R P}(Q, \nu) \mathrm{d} \sigma=\frac{2}{p} \cdot \frac{p}{12}\left(a^{\prime}-a\right) \int_{\partial \mathcal{C}} \operatorname{tr}\left(Q-Q_{\nu}\right) \mathrm{d} \sigma \\
& =\frac{\left(a^{\prime}-a\right)}{6}\left(6 \operatorname{tr}\left(Q^{2}\right)+4\right)=\left(a^{\prime}-a\right) \operatorname{tr}\left(Q^{2}\right)+\frac{2}{3}\left(a^{\prime}-a\right) .
\end{aligned}
$$

We can eliminate the constant $\frac{2}{3}\left(a^{\prime}-a\right)$ from $f_{h o m}^{R P}$, since it does not influence the minimisers of the functional $\mathcal{F}_{\varepsilon}^{R P}$, so we obtain: $f_{\text {hom }}^{R P}(Q)=\left(\underset{a^{\prime}}{ }-a\right) \operatorname{tr}\left(Q^{2}\right)$.

2. We now analyse the situation in which $f_{b}$ is of the form given by (3.4). In this situation, we choose:

$$
f_{s}^{g e n}(Q, \nu)=\frac{p}{4} \sum_{k=2}^{M} b_{k}\left(\nu \cdot Q^{k} \nu\right),
$$

where $\left(b_{k}\right)_{k \in \overline{2, M}}$ are the coefficients of the polynomial $i: \mathbb{R} \rightarrow \mathbb{R}$ of degree $M \in \mathbb{N}, M \geq 4$, defined by $i(x)=$ $\sum_{k=2}^{M} b_{k} x^{k}$, for any $x \in \mathbb{R}$, with the property that $i$ admits at least one local minimum over $\mathbb{R}$. 
In the same manner, we have

$$
f_{\text {hom }}^{\text {gen }}(Q)=\sum_{k=2}^{\max \{M, N\}} c_{k} \operatorname{tr}\left(Q^{k}\right)
$$

where, for any $k \in \overline{2, \max \{M, N\}}$, we have

$$
c_{k}=\left\{\begin{array}{l}
a_{k}+b_{k}, \text { if } 2 \leq k \leq \min \{M, N\} \\
a_{k}, \text { if } \min \{M, N\}<k \leq \max \{M, N\} \text { and } M \leq N \\
b_{k}, \text { if } \min \{M, N\}<k \leq \max \{M, N\} \text { and } M \geq N
\end{array}\right.
$$

In this case, $\mathcal{F}_{\varepsilon}$ and $\mathcal{F}_{0}$ become:

$$
\mathcal{F}_{\varepsilon}^{g e n}\left[Q_{\varepsilon}\right]:=\int_{\Omega_{\varepsilon}}\left(f_{e}\left(\nabla Q_{\varepsilon}\right)+\sum_{k=2}^{N} a_{k} \operatorname{tr}\left(Q_{\varepsilon}^{k}\right)\right) \mathrm{d} x+\frac{p}{4} \cdot \sum_{k=2}^{M} b_{k} \cdot\left(\frac{\varepsilon^{3-\alpha}}{\varepsilon-\varepsilon^{\alpha}} \int_{\partial \mathcal{N}_{\varepsilon}}\left(\nu \cdot Q_{\varepsilon}^{k} \nu\right) \mathrm{d} \sigma\right)
$$

and

$$
\mathcal{F}_{0}^{g e n}[Q]=\int_{\Omega}\left(f_{e}(\nabla Q)+\sum_{k=2}^{\max \{M, N\}} c_{k} \operatorname{tr}\left(Q^{k}\right)\right) \mathrm{d} x
$$

Theorem 3.6. Let $\left(a_{k}\right)_{k \in \overline{2, N}}$ and $\left(b_{k}\right)_{k \in \overline{2, M}}$ be such that the polynomials $h$ and $i$ defined earlier admit at least one local minimum over $\mathbb{R}$. Suppose that the assumptions $\left(A_{1}\right)-\left(A_{7}\right)$ are satisfied and also the inequalities from (3.3). Then, for any isolated $H^{1}$-local minimiser $Q_{0}$ of the functional $\mathcal{F}_{0}^{\text {gen }}$ defined by (3.18), and for $\varepsilon>0$ sufficiently small enough, there exists a sequence of local minimisers $Q_{\varepsilon}$ of the functionals $\mathcal{F}_{\varepsilon}^{\text {gen }}$, defined by (3.17), such that $E_{\varepsilon} Q_{\varepsilon} \rightarrow Q_{0}$ strongly in $H_{g}^{1}\left(\Omega, \mathcal{S}_{0}\right)$.

Proof. This theorem is a particular case of Theorem 3.2. Using once again Proposition A.8, the proof is finished.

\subsubsection{The asymmetric case $p \neq q \neq r \neq p$}

We now assume that $p, q$ and $r$ are three different real values, each greater than or equal to 1 . In this situation, the "inner particles" are not cubes anymore, but simple parallelepipeds.

We only illustrate how to proceed for the case in which we have

$$
f_{b}(Q)=a \operatorname{tr}\left(Q^{2}\right)-b \operatorname{tr}\left(Q^{3}\right)+c \operatorname{tr}\left(Q^{4}\right)=a \operatorname{tr}\left(Q^{2}\right)-b \operatorname{tr}\left(Q^{3}\right)+\frac{c}{2}\left(\operatorname{tr}\left(Q^{2}\right)\right)^{2},
$$

with $c>0$. Similar results can be obtained for the other cases in which we modify the form of $f_{b}$.

Let

$$
A=\frac{1}{3}\left(\begin{array}{cccc}
-\frac{2}{p}+\frac{1}{q}+\frac{1}{r} & 0 & 0 \\
0 & \frac{1}{p}-\frac{2}{q}+\frac{1}{r} & 0 \\
0 & 0 & \frac{1}{p}+\frac{1}{q}-\frac{2}{r}
\end{array}\right) \quad \text { and } B=\left(\begin{array}{ccc}
\frac{1}{q}+\frac{1}{r} & 0 & 0 \\
0 & \frac{1}{p}+\frac{1}{r} & 0 \\
0 & 0 & \frac{1}{p}+\frac{1}{q}
\end{array}\right)
$$


and $\omega=\frac{2}{3}\left(\frac{1}{p}+\frac{1}{q}+\frac{1}{r}\right)$. Note that $A, B$ and $\omega$ are constants depending only on the choice of $p, q$ and $r$. Moreover, we have $\operatorname{tr}(A)=0$ and $B=\omega \mathbb{I}_{3}+A$, where $\mathbb{I}_{3}$ is the $3 \times 3$ identity matrix.

Consider now

$$
f_{s}^{a s y m}(Q, \nu)=\frac{1}{2 \omega}\left(\left(a^{\prime}-a\right)\left(\nu \cdot Q^{2} \nu\right)-\left(b^{\prime}-b\right)\left(\nu \cdot Q^{3} \nu\right)+\left(c^{\prime}-c\right)\left(\nu \cdot Q^{4} \nu\right)\right),
$$

with $a^{\prime}, b^{\prime}$ and $c^{\prime}$ real parameters such that $c^{\prime}>0$ and the associated free energy functional:

$$
\mathcal{F}_{\varepsilon}^{\text {asym }}\left[Q_{\varepsilon}\right]:=\int_{\Omega}\left(f_{e}\left(\nabla Q_{\varepsilon}\right)+a \operatorname{tr}\left(Q_{\varepsilon}^{2}\right)-b \operatorname{tr}\left(Q_{\varepsilon}^{3}\right)+c \operatorname{tr}\left(Q_{\varepsilon}^{4}\right)\right) \mathrm{d} x+\frac{\varepsilon^{3-\alpha}}{\varepsilon-\varepsilon^{\alpha}} \int_{\partial \mathcal{N}_{\varepsilon}} f_{s}^{a s y m}\left(Q_{\varepsilon}, \nu\right) .
$$

We prove in the next theorem that the homogenised functional is:

$$
\begin{aligned}
f_{\text {hom }}^{\text {asym }}(Q)= & \left(\left(a^{\prime}-a\right) \operatorname{tr}\left(Q^{2}\right)-\left(b^{\prime}-b\right) \operatorname{tr}\left(Q^{3}\right)+\left(c^{\prime}-c\right) \operatorname{tr}\left(Q^{4}\right)\right) \\
& +\frac{1}{\omega}\left(\left(a^{\prime}-a\right) \operatorname{tr}\left(A \cdot Q^{2}\right)-\left(b^{\prime}-b\right) \operatorname{tr}\left(A \cdot Q^{3}\right)+\left(c^{\prime}-c\right) \operatorname{tr}\left(A \cdot Q^{4}\right)\right) .
\end{aligned}
$$

Theorem 3.7. Let $(a, b, c)$ and $\left(a^{\prime}, b^{\prime}, c^{\prime}\right)$ be two set of parameters with $c>0$ and $c^{\prime}>0$. Suppose that the assumptions $\left(A_{1}\right)-\left(A_{7}\right)$ are satisfied and also the inequalities from (3.3). Then, for $\varepsilon>0$ sufficiently small enough and for any isolated $H^{1}$-local minimiser $Q_{0}$ of the functional:

$$
\begin{aligned}
\mathcal{F}_{0}^{\text {asym }}[Q]: & =\int_{\Omega}\left(f_{e}(\nabla Q)+a^{\prime} \operatorname{tr}\left(Q^{2}\right)-b^{\prime} \operatorname{tr}\left(Q^{3}\right)+c^{\prime}\left(\operatorname{tr}\left(Q^{2}\right)\right)^{2}\right) \mathrm{d} x \\
& +\frac{1}{\omega} \int_{\Omega}\left(\left(a^{\prime}-a\right) \operatorname{tr}\left(A \cdot Q^{2}(x)\right)-\left(b^{\prime}-b\right) \operatorname{tr}\left(A \cdot Q^{3}(x)\right)+\left(c^{\prime}-c\right) \operatorname{tr}\left(A \cdot Q^{4}(x)\right)\right) \mathrm{d} x
\end{aligned}
$$

there exists a sequence of local minimisers $Q_{\varepsilon}$ of the functionals $\mathcal{F}_{\varepsilon}^{\text {asym }}$, defined by (3.20), such that $E_{\varepsilon} Q_{\varepsilon} \rightarrow Q_{0}$ strongly in $H_{g}^{1}\left(\Omega, \mathcal{S}_{0}\right)$.

Proof. We follow the same steps as in Theorem 3.4 and in Theorem 3.5, that is, we prove that relation (3.21) can be obtained using (3.1) and (3.19).

In the proof of Proposition A.8, we obtain that:

$$
\int_{\mathcal{C}^{x}} \nu \cdot Q^{k} \nu \mathrm{d} \sigma=2 q_{11, k}, \quad \int_{\mathcal{C}^{y}} \nu \cdot Q^{k} \nu \mathrm{d} \sigma=2 q_{22, k} \quad \text { and } \quad \int_{\mathcal{C}^{z}} \nu \cdot Q^{k} \nu \mathrm{d} \sigma=2 q_{33, k},
$$

for any $k \in \mathbb{N}, k \neq 0$, where $q_{i j, k}$ is the $i j$-th component of $Q^{k}$, from which we get:

$$
\begin{aligned}
& \int_{\mathcal{C}^{x}} f_{s}^{a s y m}(Q, \nu) \mathrm{d} \sigma=\frac{1}{\omega}\left(\left(a^{\prime}-a\right) q_{11,2}-\left(b^{\prime}-b\right) q_{11,3}+\left(c^{\prime}-c\right) q_{11,4}\right) \\
& \int_{\mathcal{C}^{y}} f_{s}^{a s y m}(Q, \nu) \mathrm{d} \sigma=\frac{1}{\omega}\left(\left(a^{\prime}-a\right) q_{22,2}-\left(b^{\prime}-b\right) q_{22,3}+\left(c^{\prime}-c\right) q_{22,4}\right) \\
& \int_{\mathcal{C}^{z}} f_{s}^{a s y m}(Q, \nu) \mathrm{d} \sigma=\frac{1}{\omega}\left(\left(a^{\prime}-a\right) q_{33,2}-\left(b^{\prime}-b\right) q_{33,3}+\left(c^{\prime}-c\right) q_{33,4}\right) .
\end{aligned}
$$

Using now (3.1), we obtain:

$$
f_{\text {hom }}^{\text {asym }}(Q)=\frac{1}{\omega}\left(a^{\prime}-a\right)\left(q_{11,2}\left(\frac{1}{q}+\frac{1}{r}\right)+q_{22,2}\left(\frac{1}{p}+\frac{1}{r}\right)+q_{33,2}\left(\frac{1}{p}+\frac{1}{q}\right)\right)
$$




$$
\begin{aligned}
& -\frac{1}{\omega}\left(b^{\prime}-b\right)\left(q_{11,3}\left(\frac{1}{q}+\frac{1}{r}\right)+q_{22,3}\left(\frac{1}{p}+\frac{1}{r}\right)+q_{33,3}\left(\frac{1}{p}+\frac{1}{q}\right)\right) \\
& +\frac{1}{\omega}\left(c^{\prime}-c\right)\left(q_{11,4}\left(\frac{1}{q}+\frac{1}{r}\right)+q_{22,4}\left(\frac{1}{p}+\frac{1}{r}\right)+q_{33,4}\left(\frac{1}{p}+\frac{1}{q}\right)\right)
\end{aligned}
$$

which we can see as:

$$
f_{\text {hom }}^{a s y m}(Q)=\frac{1}{\omega}\left(\left(a^{\prime}-a\right) \operatorname{tr}\left(B \cdot Q^{2}\right)-\left(b^{\prime}-b\right) \operatorname{tr}\left(B \cdot Q^{3}\right)+\left(c^{\prime}-c\right) \operatorname{tr}\left(B \cdot Q^{4}\right)\right)
$$

and since $B=\omega \mathbb{I}_{3}+A$, we obtain:

$$
\begin{aligned}
f_{\text {hom }}^{\text {asym }}(Q) & =\left(\left(a^{\prime}-a\right) \operatorname{tr}\left(Q^{2}\right)-\left(b^{\prime}-b\right) \operatorname{tr}\left(Q^{3}\right)+\left(c^{\prime}-c\right) \operatorname{tr}\left(Q^{4}\right)\right) \\
& +\frac{1}{\omega}\left(\left(a^{\prime}-a\right) \operatorname{tr}\left(A \cdot Q^{2}\right)-\left(b^{\prime}-b\right) \operatorname{tr}\left(A \cdot Q^{3}\right)+\left(c^{\prime}-c\right) \operatorname{tr}\left(A \cdot Q^{4}\right)\right),
\end{aligned}
$$

from which we conclude.

Remark 3.8. We have obtained in this case a part which is exactly the same as in the case in which we have cubic symmetry, but also three terms of the form $\operatorname{tr}\left(A \cdot Q^{k}\right)$ which describe a new preferred alignment of the liquid crystal particles inside of the domain, given by the loss of the cubic symmetry of the scaffold.

\section{Properties of the functional $\mathcal{F}_{\varepsilon}$}

\subsection{Analytical tools: trace and extension}

The main result of this subsection consists on a $L^{p}$ inequality, which is adapted from Lemma 3.1. from [13], because our scaffold now consists on inter-connected particles and the interaction between the liquid crystal and the cubic microlattice happens only up to five faces of the particles of the scaffold.

In the following, given a set $\mathcal{P} \subset \mathbb{R}^{2}$ and a real number $a>0$, we define $a \mathcal{P}=\{a x: x \in \mathcal{P}\}$.

Lemma 4.1. Let $\mathcal{P} \subseteq \mathbb{R}^{2}$ be a compact, convex set whose interior contains the origin. Let a and $b$ be positive numbers such that $a<b$. Then there exists a bijective, Lipschitz map $\phi: b \mathcal{P} \backslash a \mathcal{P} \rightarrow \bar{B}_{b} \backslash \bar{B}_{a}$ that has a Lipschitz inverse and satisfies

$$
\|\nabla \phi\|_{L^{\infty}(b \mathcal{P} \backslash a \mathcal{P})}+\left\|\nabla\left(\phi^{-1}\right)\right\|_{L^{\infty}\left(\bar{B}_{b} \backslash \bar{B}_{a}\right)} \leq C(\mathcal{P}),
$$

where $C(\mathcal{P})$ is a positive constant that depends only on $\mathcal{P}$ and neither on a nor $b$.

The proof of Lemma 4.1 follows the same steps as Lemma 3.2. from [13], the only difference being that now we are in the case of $\mathbb{R}^{2}$ instead of $\mathbb{R}^{3}$.

Lemma 4.2. Let $\mathcal{P} \subseteq \mathbb{R}^{2}$ be a compact, convex set whose interior contains the origin and $n \in[2,4]$. Then, there exists $C=C(\mathcal{P}, \phi)>0$, such that for any $0<a \leq b$ and any $u \in H^{1}(b \mathcal{P} \backslash a \mathcal{P})$, there holds

$$
\oint_{\partial(a \mathcal{P})}|u|^{n} \mathrm{~d} s \lesssim \frac{2 a C}{b^{2}-a^{2}} \int_{b \mathcal{P} \backslash a \mathcal{P}}|u|^{n} d x+\frac{n C}{2} \int_{b \mathcal{P} \backslash a \mathcal{P}}\left(|u|^{2 n-2}+|\nabla u|^{2}\right) \mathrm{d} x,
$$

where $\oint$ represents the curvilinear integral in $\mathbb{R}^{2}$. 
Proof. Using Lemma 4.1, we can restrict without loss of generality to the case in which $\mathcal{P}=\bar{B}_{1}$, which is the two dimensional unit disk, centered in origin. Then $\partial B_{\tau}=\left\{x \in \mathbb{R}^{2}:|x|=\tau\right\}=\{(\rho, \theta): \rho=\tau, \theta \in[0,2 \pi]\}$, for any $\tau>0$, and we can write, for any $\rho \in[a, b]$ and any $\theta \in[0,2 \pi]$ :

$$
\begin{aligned}
|u|^{n}(a, \theta) & =|u|^{n}(\rho, \theta)-\int_{a}^{\rho} \partial_{\tau}\left(|u|^{n}\right)(\tau, \theta) \mathrm{d} \tau \\
& \leq|u|^{n}(\rho, \theta)+n \int_{a}^{\rho}\left(|u|^{n-1} \cdot\left|\partial_{\tau} u\right|\right)(\tau, \theta) \mathrm{d} \tau \\
& \leq|u|^{n}(\rho, \theta)+\frac{n}{2} \int_{a}^{\rho}\left(|u|^{2 n-2}+\left|\partial_{\tau} u\right|^{2}\right)(\tau, \theta) \mathrm{d} \tau \\
|u|^{n}(a, \theta) & \leq|u|^{n}(\rho, \theta)+\frac{n}{2} \int_{a}^{b}\left(|u|^{2 n-2}+|\nabla u|^{2}\right)(\tau, \theta) \mathrm{d} \tau
\end{aligned}
$$

If we multiply both sides by $\rho$ and integrate over $[a, b]$ with respect to $\rho$, we get:

$$
\begin{aligned}
& |u|^{n}(a, \theta) \int_{a}^{b} \rho \mathrm{d} \rho \leq \int_{a}^{b}|u|^{n}(\rho, \theta) \cdot \rho \mathrm{d} \rho+\frac{n}{2} \int_{a}^{b} \rho \mathrm{d} \rho \int_{a}^{b}\left(|u|^{2 n-2}+|\nabla u|^{2}\right)(\tau, \theta) \mathrm{d} \tau \\
& \frac{b^{2}-a^{2}}{2}|u|^{n}(a, \theta) \leq \int_{a}^{b}|u|^{n}(\rho, \theta) \cdot \rho \mathrm{d} \rho+\frac{n\left(b^{2}-a^{2}\right)}{4} \int_{a}^{b}\left(|u|^{2 n-2}+|\nabla u|^{2}\right)(\tau, \theta) \mathrm{d} \tau .
\end{aligned}
$$

Since for any $\tau \in[a, b]$ we have $\tau>a$, then:

$$
\frac{b^{2}-a^{2}}{2 a}|u|^{n}(a, \theta) \cdot a \leq \int_{a}^{b}|u|^{n}(\rho, \theta) \cdot \rho \mathrm{d} \rho+\frac{n\left(b^{2}-a^{2}\right)}{4 a} \int_{a}^{b}\left(|u|^{2 n-2}+|\nabla u|^{2}\right)(\tau, \theta) \cdot \tau \mathrm{d} \tau .
$$

Now we integrate with respect to $\theta$ over $[0,2 \pi]$ and we get:

$$
\begin{aligned}
\frac{b^{2}-a^{2}}{2 a} \int_{0}^{2 \pi}|u|^{n}(a, \theta) \cdot a \mathrm{~d} \theta & \leq \int_{0}^{2 \pi} \int_{a}^{b}|u|^{n}(\rho, \theta) \cdot \rho \mathrm{d} \rho \mathrm{d} \theta+\frac{n\left(b^{2}-a^{2}\right)}{4 a} \int_{0}^{2 \pi} \int_{a}^{b}\left(|u|^{2 n-2}+|\nabla u|^{2}\right)(\tau, \theta) \cdot \tau \mathrm{d} \tau \mathrm{d} \theta \\
\frac{b^{2}-a^{2}}{2 a} \oint_{\partial B_{a}}|u|^{n} \mathrm{~d} s & \leq \int_{B_{b} \backslash B_{a}}|u|^{n} \mathrm{~d} x+\frac{n\left(b^{2}-a^{2}\right)}{4 a} \int_{B_{b} \backslash B_{a}}\left(|u|^{2 n-2}+|\nabla u|^{2}\right) \mathrm{d} x
\end{aligned}
$$

therefore

$$
\oint_{\partial B_{a}}|u|^{n} \mathrm{~d} s \leq \frac{2 a}{b^{2}-a^{2}} \int_{B_{b} \backslash B_{a}}|u|^{n} \mathrm{~d} x+\frac{n}{2} \int_{B_{b} \backslash B_{a}}\left(|u|^{2 n-2}+|\nabla u|^{2}\right) \mathrm{d} x .
$$

If we apply now the Lipschitz homeomorphism $\phi$ defined by Lemma 4.1, the conclusion follows.

Lemma 4.3. For any $Q \in H^{1}\left(\Omega_{\varepsilon}, \mathcal{S}_{0}\right)$ and any $n \in[2,4]$, there holds:

$$
\frac{\varepsilon^{3}}{\varepsilon^{\alpha}\left(\varepsilon-\varepsilon^{\alpha}\right)} \int_{\partial \mathcal{N}_{\varepsilon}^{\mathcal{T}}}|Q|^{n} \mathrm{~d} \sigma \lesssim \frac{n}{2} \cdot \frac{\varepsilon^{2-\alpha}}{1-\varepsilon^{\alpha-1}} \int_{\Omega_{\varepsilon}}\left(|Q|^{2 n-2}+|\nabla Q|^{2}\right) \mathrm{d} x+\frac{1}{2\left(1-\varepsilon^{\alpha-1}\right)^{2}} \int_{\Omega_{\varepsilon}}|Q|^{n} \mathrm{~d} x
$$


Proof. Let $I_{\varepsilon}[Q]=\frac{\varepsilon^{3}}{\varepsilon^{\alpha}\left(\varepsilon-\varepsilon^{\alpha}\right)} \int_{\partial \mathcal{N}_{\varepsilon}^{\mathcal{T}}}|Q|^{n} \mathrm{~d} \sigma$ and

$$
I_{\varepsilon}^{X}[Q]=\frac{\varepsilon^{3}}{\varepsilon^{\alpha}\left(\varepsilon-\varepsilon^{\alpha}\right)} \sum_{k=1}^{X_{\varepsilon}} \int_{\mathcal{T}_{x}^{k}}|Q|^{n} \mathrm{~d} \sigma .
$$

Let $\overline{e_{1}}=(1,0,0)^{T}, \overline{e_{2}}=(0,1,0)^{T}, \overline{e_{3}}=(0,0,1)^{T}$ and $k \in \overline{1, X_{\varepsilon}}$. Then, according to the definitions from Appendix A.1, $y_{\varepsilon}^{x, k}$ is the center of the "connecting parallelepiped" $\mathcal{P}_{\varepsilon}^{x, k}$ with the "contact" faces $\mathcal{T}_{x}^{k}$. If this parallelepiped is sufficiently far away from the boundary of $\Omega$, then Figure 3 shows a cross section of a neighbourhood of $\mathcal{P}_{\varepsilon}^{x, k}$, surrounding $\mathcal{T}_{x}^{k}$, a section which is parallel to the $y O z$ plane and which is passing through $y_{\varepsilon}^{x, k}+\delta \overline{e_{1}}$, where $\delta \in I_{p}:=\left[-\frac{p \varepsilon-\varepsilon^{\alpha}}{2 p}, \frac{p \varepsilon-\varepsilon^{\alpha}}{2 p}\right]$. Nevertheles, if the parallelepiped $\mathcal{P}_{\varepsilon}^{x, k}$ is close to $\partial \Omega$, then the same argument will work, since we have relations (2.5) and (2.8).

Let $\mathcal{T}_{x}^{k}(\delta)$ be

$$
\mathcal{T}_{x}^{k}(\delta)=\left\{y_{\varepsilon}^{x, k}+\delta \overline{e_{1}}+y \overline{e_{2}}+z \overline{e_{3}} \mid-\frac{\varepsilon^{\alpha}}{2 q} \leq y \leq \frac{\varepsilon^{\alpha}}{2 q} ;-\frac{\varepsilon^{\alpha}}{2 r} \leq z \leq \frac{\varepsilon^{\alpha}}{2 r}\right\}
$$

which represents the centered white rectangle from Figure 3.

Let $\mathcal{V}_{x}^{k}(\delta)$ be

$$
\mathcal{V}_{x}^{k}(\delta)=\left\{y_{\varepsilon}^{x, k}+\delta \overline{e_{1}}+y \overline{e_{2}}+z \overline{e_{3}} \mid-\varepsilon+\frac{\varepsilon^{\alpha}}{2 q} \leq y \leq \varepsilon-\frac{\varepsilon^{\alpha}}{2 q} ;-\varepsilon+\frac{\varepsilon^{\alpha}}{2 r} \leq z \leq \varepsilon-\frac{\varepsilon^{\alpha}}{2 r}\right\} \backslash \mathcal{T}_{x}^{k}(\delta),
$$

which represents the darker shaded area from Figure 3, containing only liquid crystal particles, that is $\mathcal{V}_{x}^{k}(\delta) \subset$ $\Omega_{\varepsilon}$, for any $\delta \in I_{p}$.

In our case, $\mathcal{V}_{x}^{k}(\delta)$ plays the role of $b \mathcal{P} \backslash a \mathcal{P}$ from Lemma 4.3.

If for every $\delta \in I_{p}$, we apply the translation $y_{\varepsilon}^{k}+\delta \overline{e_{1}}$ to the origin of the system, then for

$$
\mathcal{P}=\{0\} \times\left[-\frac{1}{2 q}, \frac{1}{2 q}\right] \times\left[-\frac{1}{2 r}, \frac{1}{2 r}\right]
$$

we can choose $a=\varepsilon^{\alpha}$, therefore $\varepsilon^{\alpha} \mathcal{P}=\mathcal{T}_{x}^{k}(\delta)$. In order to choose $b$, we assume: $\frac{b}{2 r} \leq \varepsilon-\frac{\varepsilon^{\alpha}}{2 r}$ and $\frac{b}{2 q} \leq \varepsilon-\frac{\varepsilon^{\alpha}}{2 q}$, that is: $b \leq 2 q \varepsilon-\varepsilon^{\alpha}$ and $b \leq 2 r \varepsilon-\varepsilon^{\alpha}$. Since $p, q, r \geq 1$, we can choose $b=2 \varepsilon-\varepsilon^{\alpha}$. In this way, we have $b \mathcal{P} \backslash a \mathcal{P} \subset \mathcal{V}_{x}^{k}(\delta)$ and we also have $b \geq a \Leftrightarrow 2 \varepsilon-\varepsilon^{\alpha} \geq \varepsilon^{\alpha} \Leftrightarrow \alpha \geq 1$.

Therefore, we can apply Lemma 4.2 for $Q$ with $a=\varepsilon^{\alpha}, b=2 \varepsilon-\varepsilon^{\alpha}$ and $\mathcal{P}$ defined as before, hence:

$$
\oint_{\partial \mathcal{T}_{x}^{k}(\delta)}|Q|^{n} \mathrm{~d} s \lesssim \frac{2 \varepsilon^{\alpha}}{\left(2 \varepsilon-\varepsilon^{\alpha}\right)^{2}-\varepsilon^{2 \alpha}} \int_{b \mathcal{P} \backslash \mathcal{T}_{x}^{k}(\delta)}|Q|^{n} \mathrm{~d} x+\frac{n}{2} \int_{b \mathcal{P} \backslash \mathcal{T}_{x}^{k}(\delta)}\left(|Q|^{2 n-2}+|\nabla Q|^{2}\right) \mathrm{d} x
$$

and since $b \mathcal{P} \subset \mathcal{V}_{x}^{k}(\delta)$, we have:

$$
\oint_{\partial \mathcal{T}_{x}^{k}(\delta)}|Q|^{n} \mathrm{~d} s \lesssim \frac{\varepsilon^{\alpha}}{2 \varepsilon\left(\varepsilon-\varepsilon^{\alpha}\right)} \int_{\mathcal{V}_{x}^{k}(\delta)}|Q|^{n} \mathrm{~d} x+\frac{n}{2} \int_{\mathcal{V}_{x}^{k}(\delta)}\left(|Q|^{2 n-2}+|\nabla Q|^{2}\right) \mathrm{d} x
$$




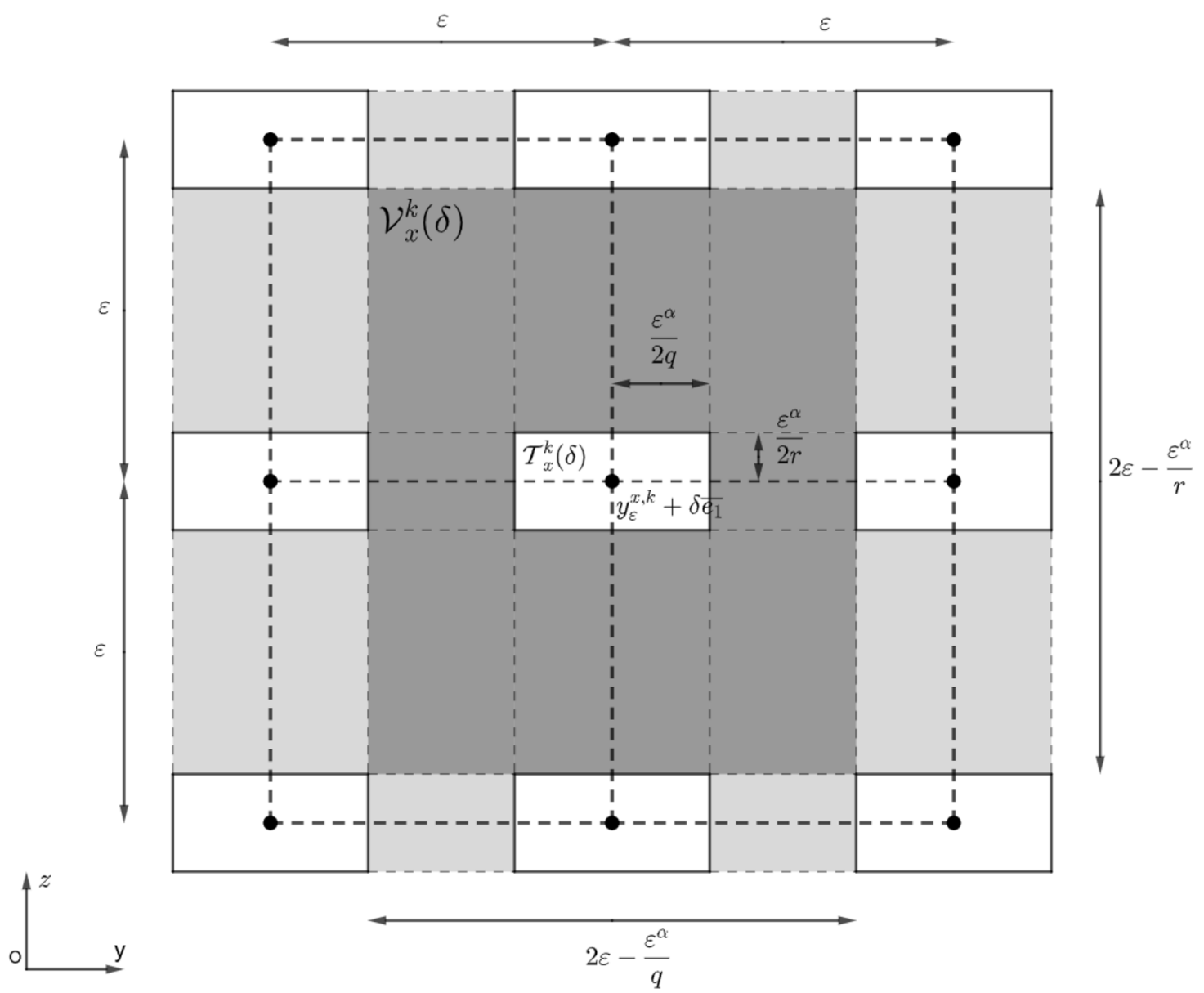

Figure 3. Cross section of the scaffold, parallel to $y O z$ plane, passing through $y_{\varepsilon}^{x, k}+\delta \overline{e_{1}}$. The gray shaded areas represent the liquid crystal and the white rectangles represent the sections of the parts of the scaffold nearby.

for every $\delta \in I_{p}$. Integrating now with respect to $\delta$ over $I_{p}$, we get:

$$
\begin{aligned}
\int_{I_{p}}\left(\oint_{\partial \mathcal{T}_{x}^{k}(\delta)}|Q|^{n} \mathrm{~d} s\right) \mathrm{d} \delta & \lesssim \frac{\varepsilon^{\alpha}}{2 \varepsilon\left(\varepsilon-\varepsilon^{\alpha}\right)} \int_{I_{p}}\left(\int_{\mathcal{V}_{x}^{k}(\delta)}|Q|^{n} \mathrm{~d} x\right) \mathrm{d} \delta+\frac{n}{2} \int_{I_{p}}\left(\int_{\mathcal{V}_{x}^{k}(\delta)}\left(|Q|^{2 n-2}+|\nabla Q|^{2}\right) \mathrm{d} x\right) \mathrm{d} \delta \\
\int_{\mathcal{T}_{x}^{k}}|Q|^{n} \mathrm{~d} \sigma & \lesssim \frac{\varepsilon^{\alpha}}{2 \varepsilon\left(\varepsilon-\varepsilon^{\alpha}\right)} \int_{\mathcal{U}_{x}^{k}}|Q|^{n} \mathrm{~d} x+\frac{n}{2} \int_{\mathcal{U}_{x}^{k}}\left(|Q|^{2 n-2}+|\nabla Q|^{2}\right) \mathrm{d} x,
\end{aligned}
$$

where $\mathcal{U}_{x}^{k}:=\bigcup_{\delta \in I_{p}} \mathcal{V}_{x}^{k}(\delta) \subset \Omega_{\varepsilon}$ is now a three dimensional object. Hence:

$$
\frac{\varepsilon^{3-\alpha}}{\varepsilon-\varepsilon^{\alpha}} \int_{\mathcal{T}_{x}^{k}}|Q|^{n} \mathrm{~d} \sigma \lesssim \frac{n}{2} \cdot \frac{\varepsilon^{2-\alpha}}{1-\varepsilon^{\alpha-1}} \int_{\mathcal{U}_{x}^{k}}\left(|Q|^{2 n-2}+|\nabla Q|^{2}\right) \mathrm{d} x+\frac{1}{2\left(1-\varepsilon^{\alpha-1}\right)^{2}} \int_{\mathcal{U}_{x}^{k}}|Q|^{n} \mathrm{~d} x
$$

Repeating the same argument for all the other "connecting parallelepipeds" of the scaffold and considering the fact that parts of $\mathcal{U}_{x}^{k}$ are added only up to four times (by constructing the same sets for the nearby "connecting parallelepipeds" from the scaffold), then the conclusion follows. 
Since we are interested in the homogenised material, it is useful to consider maps defined on the entire $\Omega$ and for this we use the harmonic extension operator $E_{\varepsilon}: H_{g}^{1}\left(\Omega_{\varepsilon}, \mathcal{S}_{0}\right) \rightarrow H_{g}^{1}\left(\Omega, \mathcal{S}_{0}\right)$, defined as follows: for $Q \in H_{g}^{1}\left(\Omega_{\varepsilon}, \mathcal{S}_{0}\right)$, we take $E_{\varepsilon} Q \equiv Q$ in $\Omega_{\varepsilon}$ and inside $\mathcal{N}_{\varepsilon}, E_{\varepsilon} Q$ solves the following PDE:

$$
\begin{cases}\Delta E_{\varepsilon} Q=0 & \text { in } \mathcal{N}_{\varepsilon} \\ E_{\varepsilon} Q \equiv Q & \text { on } \partial \mathcal{N}_{\varepsilon}\end{cases}
$$

Since $\mathcal{N}_{\varepsilon}$ has a Lipschitz boundary, we can apply Theorem 4.19 from [15] and see that there exists a unique solution $E_{\varepsilon} Q \in H^{1}\left(\mathcal{N}_{\varepsilon}\right)$ to the problem (4.1). Hence the operator $E_{\varepsilon}$ is well defined. Moreover, from (4.1), we can see that $E_{\varepsilon} Q$ verifies:

$$
\left\|\nabla E_{\varepsilon} Q\right\|_{L^{2}\left(\mathcal{N}_{\varepsilon}\right)}=\min \left\{\|\nabla u\|_{L^{2}\left(\mathcal{N}_{\varepsilon}\right)} \mid u \in H^{1}\left(\mathcal{N}_{\varepsilon}\right), u=Q \text { on } \partial \mathcal{N}_{\varepsilon}\right\}
$$

Our aim is now to prove that the extension operator $E_{\varepsilon}$ is uniformly bounded with respect to $\varepsilon>0$. More specifically, we prove that the following lemma holds.

Lemma 4.4. There exists a constant $C>0$ such that $\left\|\nabla E_{\varepsilon} Q\right\|_{L^{2}(\Omega)} \leq C\|\nabla Q\|_{L^{2}\left(\Omega_{\varepsilon}\right)}$ for any $\varepsilon \in\left(0, \varepsilon_{0}\right)$, where $\varepsilon_{0}$ is suitably small enough, and for any $Q \in H_{g}^{1}\left(\Omega_{\varepsilon}, \mathcal{S}_{0}\right)$.

Proof. By Appendix A.3, we know that there exists $v \in H^{1}(\Omega)$ such that:

$$
\left\{\begin{array}{l}
v \equiv Q \text { in } \Omega_{\varepsilon} \\
v=Q \text { on } \partial \mathcal{N}_{\varepsilon} \\
\|\nabla v\|_{L^{2}(\Omega)} \lesssim\|\nabla Q\|_{L^{2}\left(\Omega_{\varepsilon}\right)} .
\end{array}\right.
$$

Using relation (4.2), we see that

$$
\left\|\nabla E_{\varepsilon} Q\right\|_{L^{2}\left(\mathcal{N}_{\varepsilon}\right)} \leq\|\nabla v\|_{L^{2}\left(\mathcal{N}_{\varepsilon}\right)}
$$

and because $E_{\varepsilon} Q \equiv Q$ in $\Omega_{\varepsilon}$, we have $E_{\varepsilon} Q \equiv v \equiv Q$ in $\Omega_{\varepsilon}$ and therefore:

$$
\left\|\nabla E_{\varepsilon} Q\right\|_{L^{2}(\Omega)} \leq\|\nabla v\|_{L^{2}(\Omega)} \lesssim\|\nabla Q\|_{L^{2}\left(\Omega_{\varepsilon}\right)} .
$$

\subsection{Zero contribution from the surface terms depending on the "inner parallelepipeds"}

In order to describe the surface energy, we need a better description of $\partial \mathcal{N}_{\varepsilon}$, therefore we analyse what faces from every parallelepiped constructed are in contact with the liquid crystal. More precisely, the liquid crystal is in contact with the scaffold:

- on only four of the six faces of the "connecting parallelepipeds", centered in points from $\mathcal{Y}_{\varepsilon}$, that is, on every $\mathcal{T}_{x}^{k}, \mathcal{T}_{y}^{l}$ and $\mathcal{T}_{z}^{m}$, defined in (A.6), (A.11) and (A.16);

- only on the edges of some of the "inner parallelepipeds", centered in some of the points from $\mathcal{X}_{\varepsilon}$, parallelepipeds which are not close to the boundary of $\Omega$ - we are reffering here to the "inner particles" which are not "visible" in Figure 1 - in this case, the interaction is neglected and let

$$
N_{\varepsilon, 1}=\text { the total number of parallelepipeds from this case; }
$$


- on at most five of the six faces of some of the "inner parallelepipeds", centered in some of the points from $\mathcal{X}_{\varepsilon}$, parallelepipeds which are close to the boundary of $\Omega$ - we are reffering here to the "inner particles" which are "visible" in Figure 1 - in this case, let

$$
N_{\varepsilon, 2}=\text { the total number of parallelepipeds from this case; }
$$

and let

$$
\begin{gathered}
\mathcal{S}^{i}=\text { the union of all the rectangles (at most five in this case) that } \\
\text { are in contact with the liquid crystal material, }
\end{gathered}
$$

for any $i \in \overline{1, N_{\varepsilon, 2}}$.

From relations (4.3) and (4.4), we have $N_{\varepsilon}=N_{\varepsilon, 1}+N_{\varepsilon, 2}$. Using (A.6), (A.11), (A.16) and (4.5), we can write $\partial \mathcal{N}_{\varepsilon}=\partial \mathcal{N}_{\varepsilon}^{\mathcal{S}} \cup \partial \mathcal{N}_{\varepsilon}^{\mathcal{T}}$, where:

$$
\partial \mathcal{N}_{\varepsilon}^{\mathcal{S}}=\left(\bigcup_{i=1}^{N_{\varepsilon, 2}} \mathcal{S}^{i}\right) \text { and } \partial \mathcal{N}_{\varepsilon}^{\mathcal{T}}=\left(\bigcup_{k=1}^{X_{\varepsilon}} \mathcal{T}_{x}^{k}\right) \cup\left(\bigcup_{l=1}^{Y_{\varepsilon}} \mathcal{T}_{y}^{l}\right) \cup\left(\bigcup_{m=1}^{Z_{\varepsilon}} \mathcal{T}_{z}^{m}\right)
$$

Let $J_{\varepsilon}[Q]$ be the surface energy term from (1.1) and let us split this term into two parts:

$$
J_{\varepsilon}[Q]=J_{\varepsilon}^{\mathcal{S}}[Q]+J_{\varepsilon}^{\mathcal{T}}[Q],
$$

where

$$
J_{\varepsilon}^{\mathcal{S}}[Q]=\frac{\varepsilon^{3-\alpha}}{\varepsilon-\varepsilon^{\alpha}} \int_{\partial \mathcal{N}_{\varepsilon}^{\mathcal{S}}} f_{\mathcal{S}}(Q, \nu) \mathrm{d} \sigma=\frac{\varepsilon^{3-\alpha}}{\varepsilon-\varepsilon^{\alpha}} \sum_{i=1}^{N_{\varepsilon, 2}} \int_{\mathcal{S}^{i}} f_{S}(Q, \nu) \mathrm{d} \sigma,
$$

using (4.6), and

$$
J_{\varepsilon}^{\mathcal{T}}[Q]=\frac{\varepsilon^{3-\alpha}}{\varepsilon-\varepsilon^{\alpha}} \int_{\partial \mathcal{N}_{\varepsilon}^{\mathcal{T}}} f_{s}(Q, \nu) \mathrm{d} \sigma,
$$

which can be also expressed using (4.6) as

$$
J_{\varepsilon}^{\mathcal{T}}[Q]=J_{\varepsilon}^{X}[Q]+J_{\varepsilon}^{Y}[Q]+J_{\varepsilon}^{Z}[Q],
$$

where:

$$
\left\{\begin{array}{l}
J_{\varepsilon}^{X}[Q]=\frac{\varepsilon^{3-\alpha}}{\varepsilon-\varepsilon^{\alpha}} \sum_{k=1}^{X_{\varepsilon}} \int_{\mathcal{T}_{x}^{k}} f_{s}(Q, \nu) \mathrm{d} \sigma \\
J_{\varepsilon}^{Y}[Q]=\frac{\varepsilon^{3-\alpha}}{\varepsilon-\varepsilon^{\alpha}} \sum_{l=1}^{Y_{\varepsilon}} \int_{\mathcal{T}_{y}^{l}} f_{s}(Q, \nu) \mathrm{d} \sigma \\
J_{\varepsilon}^{Z}[Q]=\frac{\varepsilon^{3-\alpha}}{\varepsilon-\varepsilon^{\alpha}} \sum_{m=1}^{Z_{\varepsilon}} \int_{\mathcal{T}_{z}^{m}} f_{s}(Q, \nu) \mathrm{d} \sigma .
\end{array}\right.
$$


In this section, we prove that the surface term $J_{\varepsilon}^{\mathcal{S}}$ has a negligible contribution to the homogenised material, that is $J_{\varepsilon}^{\mathcal{S}}[Q] \rightarrow 0$ as $\varepsilon \rightarrow 0$, for any $Q \in H_{g}^{1}\left(\Omega, \mathcal{S}_{0}\right)$, since we can use the extension operator $E_{\varepsilon}$ defined in the previous subsection.

We start by proving if $Q: \bar{\Omega} \rightarrow \mathcal{S}_{0}$ is a bounded, Lipschitz map, then $J_{\varepsilon}^{\mathcal{S}}[Q] \rightarrow 0$ as $\varepsilon \rightarrow 0$ and then, by a density argument, for all $Q \in H_{g}^{1}\left(\Omega, \mathcal{S}_{0}\right)$.

Lemma 4.5. Let $Q: \bar{\Omega} \rightarrow \mathcal{S}_{0}$ be a bounded, Lipschitz map. Then $J_{\varepsilon}^{\mathcal{S}}[Q] \rightarrow 0$, as $\varepsilon \rightarrow 0$, where $J_{\varepsilon}^{\mathcal{S}}$ is defined in (4.7) and in (4.8).

Proof. By (4.8), we have:

$$
\begin{aligned}
\left|\frac{\varepsilon^{3-\alpha}}{\varepsilon-\varepsilon^{\alpha}} \sum_{i=1}^{N_{\varepsilon, 2}} \int_{\mathcal{S}^{i}} f_{s}(Q(t), \nu) \mathrm{d} \sigma(t)\right| & \leq \frac{\varepsilon^{3-\alpha}}{\varepsilon-\varepsilon^{\alpha}} \sum_{i=1}^{N_{\varepsilon, 2}} \int_{\mathcal{S}^{i}}\left|f_{s}(Q(t), \nu)\right| \mathrm{d} \sigma(t) \\
& \leq \frac{C \varepsilon^{3-\alpha}}{\varepsilon-\varepsilon^{\alpha}} \sum_{i=1}^{N_{\varepsilon, 2}} \int_{\mathcal{S}^{i}}\left(|Q|^{4}(t)+1\right) \mathrm{d} \sigma(t) \\
& \leq \frac{C \varepsilon^{3-\alpha}}{\varepsilon-\varepsilon^{\alpha}} \sum_{i=1}^{N_{\varepsilon, 2}} \int_{\partial \mathcal{C}_{\varepsilon}^{i}}\left(|Q|^{4}(t)+1\right) \mathrm{d} \sigma(t) \\
& \leq \frac{\varepsilon^{3+\alpha}}{\varepsilon-\varepsilon^{\alpha}} \cdot \frac{2 C(p+q+r)}{p q r} \cdot\left(\|Q\|_{L^{\infty}(\bar{\Omega})}^{4}+1\right) \cdot \sum_{i=1}^{N_{\varepsilon, 2}} \int_{\partial \mathcal{C}} \mathrm{d} \sigma(t) \\
& \leq \frac{\varepsilon^{3+\alpha}}{\varepsilon-\varepsilon^{\alpha}} \cdot\left(\|Q\|_{L^{\infty}(\bar{\Omega})}^{4}+1\right) \cdot \frac{2 C(p+q+r)}{p q r} \cdot \sigma(\partial \mathcal{C}) \cdot N_{\varepsilon, 2},
\end{aligned}
$$

where $\partial \mathcal{C}$ represents the surface of the model particle $\mathcal{C}$ defined in $(2.2), \mathcal{C}_{\varepsilon}^{i}$ represents the "inner parallelepipeds" constructed in relation (2.7), $N_{\varepsilon, 2}$ is defined in (4.4) and $C$ is the $\varepsilon$-independent constant given from the inequality that states that $f_{s}$ has a quartic growth in $Q$, which can be obtained from assumption $\left(A_{7}\right)$. We have also used that $Q$ is bounded on $\bar{\Omega}$. In the proof of Proposition A.4, we obtain $N_{\varepsilon, 2} \leq \frac{L_{0} l_{0}+l_{0} h_{0}+h_{0} L_{0}}{\varepsilon^{2}}$, hence:

$$
\begin{aligned}
& \left|\frac{\varepsilon^{3-\alpha}}{\varepsilon-\varepsilon^{\alpha}} \sum_{i=1}^{N_{\varepsilon, 2}} \int_{\mathcal{S}^{i}} f_{s}(Q(t), \nu) \mathrm{d} \sigma(t)\right|<C^{\prime} \cdot \frac{\varepsilon^{3+\alpha}}{\varepsilon-\varepsilon^{\alpha}} \cdot \frac{L_{0} l_{0}+L_{0} h_{0}+l_{0} h_{0}}{\varepsilon^{2}} \\
\Rightarrow & \left|\frac{\varepsilon^{3-\alpha}}{\varepsilon-\varepsilon^{\alpha}} \sum_{i=1}^{N_{\varepsilon, 2}} \int_{\mathcal{S}^{i}} f_{s}(Q(t), \nu) \mathrm{d} \sigma(t)\right|<C^{\prime \prime} \cdot \frac{\varepsilon^{\alpha}}{1-\varepsilon^{\alpha-1}} \rightarrow 0 \text { as } \varepsilon \rightarrow 0,
\end{aligned}
$$

since $\alpha \in\left(1, \frac{3}{2}\right)$, where $L_{0}, l_{0}$ and $h_{0}$ are defined in $(2.1)$ and $C^{\prime}$ and $C^{\prime \prime}$ are $\varepsilon$-independent constants.

Lemma 4.6. For any $Q \in H_{g}^{1}\left(\Omega_{\varepsilon}, \mathcal{S}_{0}\right)$, we have $J_{\varepsilon}^{\mathcal{S}}[Q] \rightarrow 0$ as $\varepsilon \rightarrow 0$.

Proof. Let $\left(Q_{j}\right)_{j \geq 1}$ be a sequence of smooth maps that converge strongly in $H_{g}^{1}\left(\Omega_{\varepsilon}, \mathcal{S}_{0}\right)$ to $Q$. By Lemma 4.5 , we have $J_{\varepsilon}^{\mathcal{S}}\left[Q_{j}\right] \rightarrow 0$ as $\varepsilon \rightarrow 0$, for any $j \geq 1$. By assumption $\left(A_{7}\right)$, we have on $\mathcal{N}_{\varepsilon}^{\mathcal{S}}$ :

$$
\begin{aligned}
\left|f_{s}\left(Q_{j}, \nu\right)-f_{s}(Q, \nu)\right| & \leq\left|Q_{j}-Q\right|\left(\left|Q_{j}\right|^{3}+|Q|^{3}+1\right) \\
& \lesssim\left|Q_{j}-Q\right|\left(\left|Q_{j}-Q\right|^{3}+|Q|^{3}+1\right)
\end{aligned}
$$




$$
\lesssim\left|Q_{j}-Q\right|^{4}+\left|Q_{j}-Q\right|\left(|Q|^{3}+1\right)
$$

Thanks to the continuity of the trace operator from $H^{1}\left(\Omega_{\varepsilon}\right)$ to $H^{1 / 2}\left(\partial \Omega_{\varepsilon}\right)$, the Sobolev embedding $H^{1 / 2}\left(\partial \Omega_{\varepsilon}\right) \hookrightarrow L^{4}\left(\partial \Omega_{\varepsilon}\right)$ and the strong convergence $Q_{j} \rightarrow Q$ in $H_{g}^{1}\left(\Omega_{\varepsilon}, \mathcal{S}_{0}\right)$, we get that $Q_{j} \rightarrow Q$ a.e. on $\partial \mathcal{N}_{\varepsilon}^{\mathcal{S}}$, since $\partial \mathcal{N}_{\varepsilon}^{\mathcal{S}} \subset \partial \Omega_{\varepsilon}$. Therefore, there exists $\psi \in L^{4}\left(\partial \mathcal{N}_{\varepsilon}^{\mathcal{S}}\right)$ such that $\left|Q_{j}-Q\right| \leq \psi$ a.e. in $\partial \mathcal{N}_{\varepsilon}^{\mathcal{S}}$ and we can write:

$$
\left|f_{s}\left(Q_{j}, \nu\right)-f_{s}(Q, \nu)\right| \lesssim \psi^{4}+\psi\left(|Q|^{3}+1\right)
$$

on $\partial \mathcal{N}_{\varepsilon}^{\mathcal{S}}$, for every $j \geq 1$.

At the same time, we have the compact Sobolev embedding $H^{1 / 2}\left(\partial \Omega_{\varepsilon}\right) \hookrightarrow L^{3}\left(\partial \Omega_{\varepsilon}\right)$, therefore $|Q|^{3}$ is in $L^{1}\left(\partial \mathcal{N}_{\mathcal{E}}^{\mathcal{S}}\right)$. Hence, the right hand side from $(4.12)$ is in $L^{1}\left(\partial \mathcal{N}_{\mathcal{E}}^{\mathcal{S}}\right)$ and we can apply the Lebesgue dominated convergence theorem and get:

$$
\lim _{j \rightarrow+\infty} \int_{\partial \mathcal{N}_{\varepsilon}^{S}}\left|f_{s}\left(Q_{j}, \nu\right)-f_{s}(Q, \nu)\right| \mathrm{d} \sigma=0
$$

for any $\varepsilon>0$ fixed.

Now, because for $\varepsilon \rightarrow 0$ we get $\left|\partial \mathcal{N}_{\varepsilon}^{\mathcal{S}}\right| \rightarrow 0$ (according to Prop. A.4) and $\frac{\varepsilon^{3-\alpha}}{\varepsilon-\varepsilon^{\alpha}} \rightarrow 0$, the conclusion follows. of:

Therefore, from now on we omit the term $J_{\varepsilon}^{\mathcal{S}}$ from the free energy functional and we only study the behaviour

$$
\mathcal{F}_{\varepsilon}^{\mathcal{T}}[Q]:=\int_{\Omega_{\varepsilon}}\left(f_{e}(\nabla Q)+f_{b}(Q)\right) \mathrm{d} x+\frac{\varepsilon^{3-\alpha}}{\varepsilon-\varepsilon^{\alpha}} \int_{\partial \mathcal{N}_{\varepsilon}^{\mathcal{T}}} f_{\mathcal{s}}(Q, \nu) \mathrm{d} \sigma
$$

which we denote simply by $\mathcal{F}_{\varepsilon}[Q]$, but we keep the same notation for surfaces generated by the scaffold.

\subsection{Equicoercivity of $\mathcal{F}_{\varepsilon}$}

Proposition 4.7. Suppose that the assumptions $\left(A_{1}\right)-\left(A_{7}\right)$ hold and also that there exists $\mu>0$ such that $f_{b}(Q) \geq \mu|Q|^{6}-C$, for any $Q \in \mathcal{S}_{0}$. Let $Q \in H_{g}^{1}\left(\Omega_{\varepsilon}, \mathcal{S}_{0}\right)$ satisfy $\mathcal{F}_{\varepsilon}[Q] \leq M$, for some $\varepsilon$-independent constant. Then there holds

$$
\int_{\Omega_{\varepsilon}}|\nabla Q|^{2} \leq C_{M}
$$

for $\varepsilon>0$ small enough and for some $C_{M}>0$ depending only on $M, f_{e}, f_{b}, f_{s}$ and $\Omega$.

Proof. Assumption $\left(A_{6}\right)$ ensures that $\left|f_{s}(Q, \nu)\right| \lesssim|Q|^{4}+1$, therefore:

$$
J_{\varepsilon}^{\mathcal{T}}[Q] \geq-C_{1} \cdot \frac{\varepsilon^{3-\alpha}}{\varepsilon-\varepsilon^{\alpha}} \int_{\partial \mathcal{N}_{\varepsilon}^{\mathcal{T}}}\left(|Q|^{4}+1\right) \mathrm{d} \sigma \geq-C_{1} \cdot \frac{\varepsilon^{3-\alpha}}{\varepsilon-\varepsilon^{\alpha}} \int_{\partial \mathcal{N}_{\varepsilon}^{\mathcal{T}}}|Q|^{4} \mathrm{~d} \sigma-C_{1} \cdot C_{s},
$$

according to Proposition A.3. Using Lemma 4.3 with $n=4$, we have:

$$
\frac{\varepsilon^{3-\alpha}}{\varepsilon-\varepsilon^{\alpha}} \int_{\partial \mathcal{N}_{\varepsilon}^{\mathcal{T}}}|Q|^{4} \mathrm{~d} \sigma \lesssim \frac{2 \varepsilon^{2-\alpha}}{\left(1-\varepsilon^{\alpha-1}\right)} \int_{\Omega_{\varepsilon}}\left(|Q|^{6}+|\nabla Q|^{2}\right) \mathrm{d} x+\frac{1}{2\left(1-\varepsilon^{\alpha-1}\right)^{2}} \int_{\Omega_{\varepsilon}}|Q|^{4} \mathrm{~d} x
$$


hence

$$
J_{\varepsilon}^{\mathcal{T}}[Q] \geq-C_{1} \cdot C_{2} \cdot \frac{2 \varepsilon^{2-\alpha}}{1-\varepsilon^{\alpha-1}} \int_{\Omega_{\varepsilon}}\left(|Q|^{6}+|\nabla Q|^{2}\right) \mathrm{d} x-C_{1} \cdot C_{2} \cdot \frac{1}{2\left(1-\varepsilon^{\alpha-1}\right)^{2}} \int_{\Omega_{\varepsilon}}|Q|^{4} \mathrm{~d} x-C_{1} \cdot C_{s} .
$$

At the same time, from the generalised version of the Hölder's inequality and from the fact that $\Omega$ is bounded, we have

$$
\left(\int_{\Omega_{\varepsilon}}|Q|^{4} \mathrm{~d} x\right)^{1 / 4} \leq\left|\Omega_{\varepsilon}\right|^{1 / 12} \cdot\left(\int_{\Omega_{\varepsilon}}|Q|^{6} \mathrm{~d} x\right)^{1 / 6} \Rightarrow \int_{\Omega_{\varepsilon}}|Q|^{4} \mathrm{~d} x<|\Omega|^{1 / 3}\left(\int_{\Omega_{\varepsilon}}|Q|^{6} \mathrm{~d} x\right)^{2 / 3}
$$

and so

$$
J_{\varepsilon}^{\mathcal{T}}[Q] \geq-C_{3} \cdot \frac{\varepsilon^{2-\alpha}}{1-\varepsilon^{\alpha-1}} \int_{\Omega_{\varepsilon}}\left(|Q|^{6}+|\nabla Q|^{2}\right) \mathrm{d} x-C_{3} \cdot \frac{1}{2\left(1-\varepsilon^{\alpha-1}\right)^{2}} \cdot|\Omega|^{1 / 3}\left(\int_{\Omega_{\varepsilon}}|Q|^{6} \mathrm{~d} x\right)^{2 / 3}-C_{3},
$$

where $C_{3}=\max \left\{C_{1} \cdot C_{2}, C_{1} \cdot C_{s}\right\}$.

Since $f_{b}(Q) \geq \mu|Q|^{6}-C, f_{e}(\nabla Q) \geq \lambda_{e}^{-1}|\nabla Q|^{2}$ (according to $\left(A_{5}\right)$ and $\left.\left(A_{6}\right)\right)$ and $\left|\Omega_{\varepsilon}\right| \leq|\Omega|$, we have:

$$
\int_{\Omega_{\varepsilon}}\left(f_{b}(Q)+f_{e}(\nabla Q)\right) \mathrm{d} x \geq \mu \int_{\Omega_{\varepsilon}}|Q|^{6} \mathrm{~d} x+\lambda_{e}^{-1} \int_{\Omega_{\varepsilon}}|\nabla Q|^{2} \mathrm{~d} x-C|\Omega|
$$

and because $\mathcal{F}_{\varepsilon}[Q] \leq M$, combining (4.13) and (4.14), we obtain

$$
\left(\lambda_{e}^{-1}-C_{3} \cdot \frac{\varepsilon^{2-\alpha}}{1-\varepsilon^{\alpha-1}}\right) \int_{\Omega_{\varepsilon}}|\nabla Q|^{2} \mathrm{~d} x \leq h_{\varepsilon}\left(\left(\int_{\Omega_{\varepsilon}}|Q|^{6} \mathrm{~d} x\right)^{1 / 3}\right)
$$

where

$$
h_{\varepsilon}(t)=t^{2} \cdot\left(C_{4}(\varepsilon)+t \cdot C_{5}(\varepsilon)\right)+C_{6},
$$

for any $t \geq 0$, with $C_{4}(\varepsilon)=\frac{C_{3} \cdot|\Omega|^{1 / 3}}{2\left(1-\varepsilon^{\alpha-1}\right)^{2}}, C_{5}(\varepsilon)=C_{3} \cdot \frac{\varepsilon^{2-\alpha}}{1-\varepsilon^{\alpha-1}}-\mu$ and $C_{6}=\left(M+C_{3}+C|\Omega|\right)$. As $\varepsilon \rightarrow 0$, we have $C_{4}(\varepsilon) \searrow \frac{C_{3} \cdot|\Omega|^{1 / 3}}{2}>0$ and $C_{5}(\varepsilon) \searrow(-\mu)<0$. Hence, for $\varepsilon>0$ small enough, we have:

$$
\frac{C_{3} \cdot|\Omega|^{1 / 3}}{2}<C_{4}(\varepsilon)<C_{3} \cdot|\Omega|^{1 / 3} \text { and }-\mu<C_{5}(\varepsilon)<-\frac{\mu}{2}<0 .
$$

Let $t_{0}(\varepsilon)$ be the solution of the equation $C_{4}(\varepsilon)+t \cdot C_{5}(\varepsilon)=0$. We prove that $h_{\varepsilon}(t)$ is bounded from above on $[0,+\infty)$. Computing the critical points of $h_{\varepsilon}$, it is easy to check that $2 t_{0}(\varepsilon) / 3$ is the point in which the function attains its maximum over $[0,+\infty)$, which is:

$$
\max \left\{h_{\varepsilon}(t): t \in[0,+\infty)\right\}=\frac{4 C_{4}^{3}(\varepsilon)}{27 C_{5}^{2}(\varepsilon)}+C_{6}<\frac{4}{27} \cdot C_{3}^{3} \cdot|\Omega| \cdot \frac{4}{\mu^{2}}+C_{6},
$$

using (4.16). Therefore, the function $h_{\varepsilon}$ is bounded from above on $[0,+\infty)$.

Using the same arguments we can see that $\lambda_{e}^{-1}-C_{3} \cdot \frac{\varepsilon^{2-\alpha}}{1-\varepsilon^{\alpha-1}}$ is also bounded from below, away from 0 , for $\varepsilon>0$ small enough, and from here the conclusion follows, based on relation (4.15). 
In the end of this subsection, we present a situation in which if $\alpha>2$, then the energy becomes unbounded from below. For simplicity, we choose the scaffold to be symmetric with $p=q=r=1$. Let us consider the following free energy functional:

$$
\mathcal{G}_{\varepsilon}(u)=\int_{\Omega_{\varepsilon}}\left(|\nabla u|^{2}+k|u|^{2 l-2}\right) \mathrm{d} x-\delta \cdot \frac{\varepsilon^{3-\alpha}}{\varepsilon-\varepsilon^{\alpha}} \int_{\partial \mathcal{N}_{\varepsilon}}|u|^{l} \mathrm{~d} \sigma
$$

where $k>0$ and $l \in(2,4)$, for $u: \Omega \rightarrow \mathbb{R}, u \in H^{1}\left(\Omega_{\varepsilon}\right)$.

Lemma 4.8. For any $l \in(2,4), 2<\alpha<\frac{4}{l-2}, k>0, \delta>0$, there holds:

$$
\inf \left\{\mathcal{G}_{\varepsilon}(u): u \in H^{1}\left(\Omega_{\varepsilon}\right), u=0 \text { on } \partial \Omega\right\} \rightarrow-\infty \quad \text { as } \varepsilon \rightarrow 0 .
$$

Proof. Let $\mathcal{C}_{3 / 2}=[-3 / 2,3 / 2]^{3}$ and we recall that $\mathcal{C}=[-1,1]^{3}$. Let us consider now $\varphi \in C_{c}^{\infty}\left(\mathcal{C}_{3 / 2}\right)$ such that $\varphi \equiv 1$ on $\mathcal{C}$.

Let us now choose an "inner parallelepiped" from the scaffold such that it is close to the boundary of $\Omega$ and such that it has less than 6 adjacent "connecting parallelepipeds". In this case, some of its faces are in contact with the nematic liquid crystal. Let us denote this "inner parallelepiped" by $\mathcal{C}_{\varepsilon}^{0}$ and its center $x_{\varepsilon}^{0}$. We can write, based on A.1, that:

$$
\mathcal{C}_{\varepsilon}^{0}=x_{\varepsilon}^{0}+\left[-\frac{\varepsilon^{\alpha}}{2}, \frac{\varepsilon^{\alpha}}{2}\right]^{3}=x_{\varepsilon}^{0}+\frac{\varepsilon^{\alpha}}{2} \mathcal{C}
$$

Let us define the following function:

$$
u_{\varepsilon}(x)=\varepsilon^{-\alpha / 2-\beta} \varphi\left(\frac{2}{\varepsilon^{\alpha}}\left(x-x_{\varepsilon}^{0}\right)\right), \forall x \in \Omega,
$$

where $\beta>0$ will be chosen later.

Let us also define:

$$
\mathcal{R}_{\varepsilon}^{0}=x_{\varepsilon}^{0}+\frac{3}{2} \cdot \frac{\varepsilon^{\alpha}}{2} \cdot \mathcal{C} .
$$

Due to assumption $\left(A_{3}\right)$ and our choice of $\alpha$, we have that $\mathcal{C}_{\varepsilon}^{0} \subset \mathcal{R}_{\varepsilon}^{0} \subset \Omega$.

From the definition of $u_{\varepsilon}$, we have that $u_{\varepsilon} \equiv 1$ in $\mathcal{C}_{\varepsilon}^{0}$. Moreover, $u_{\varepsilon} \equiv 0$ in $\Omega \backslash \mathcal{R}_{\varepsilon}^{0}$ and $u_{\varepsilon} \in H^{1}(\Omega)$.

Let us define now $\mathcal{T}_{\varepsilon}^{0}$ the faces of $\mathcal{C}_{\varepsilon}^{0}$ that are in contact with the nematic liquid crystal. Going back to the definition of the free energy functional, we have:

$$
\mathcal{G}_{\varepsilon}\left(u_{\varepsilon}\right) \leq \int_{\Omega_{\varepsilon}}\left(\left|\nabla u_{\varepsilon}\right|^{2}+\left|u_{\varepsilon}\right|^{2 l-2}\right) \mathrm{d} x-\delta \cdot \frac{\varepsilon^{3-\alpha}}{\varepsilon-\varepsilon^{\alpha}} \int_{\mathcal{T}_{\varepsilon}^{0}}\left|u_{\varepsilon}\right|^{l} \mathrm{~d} \sigma,
$$

since $\mathcal{T}_{\varepsilon}^{0} \subset \partial \mathcal{N}_{\varepsilon}$.

Using the properties of $u_{\varepsilon}$, we obtain that:

$$
\begin{aligned}
\mathcal{G}_{\varepsilon}\left(u_{\varepsilon}\right) & \leq \int_{\Omega_{\varepsilon} \cap \mathcal{R}_{\varepsilon}^{0}}\left(\left|\nabla u_{\varepsilon}\right|^{2}+\left|u_{\varepsilon}\right|^{2 l-2}\right) \mathrm{d} x-\delta \cdot \frac{\varepsilon^{3-\alpha}}{\varepsilon-\varepsilon^{\alpha}} \int_{\mathcal{T}_{\varepsilon}^{0}}\left|u_{\varepsilon}\right|^{l} \mathrm{~d} \sigma \\
& \leq \int_{\mathcal{R}_{\varepsilon}^{0} \backslash \mathcal{C}_{\varepsilon}^{0}}\left(\left|\nabla u_{\varepsilon}\right|^{2}+\left|u_{\varepsilon}\right|^{2 l-2}\right) \mathrm{d} x-\delta \cdot \varepsilon^{2-\alpha} \int_{\mathcal{T}_{\varepsilon}^{0}}\left|u_{\varepsilon}\right|^{l} \mathrm{~d} \sigma:=\overline{\mathcal{G}_{\varepsilon}}\left(u_{\varepsilon}\right),
\end{aligned}
$$


since $\Omega_{\varepsilon} \cap \mathcal{R}_{\varepsilon}^{0} \subset \mathcal{R}_{\varepsilon}^{0} \backslash \mathcal{C}_{\varepsilon}^{0}$ and, due to our choice of $\alpha$, we have that $\varepsilon / 2<\varepsilon-\varepsilon^{\alpha}<\varepsilon$, which implies that $-\frac{\varepsilon^{3-\alpha}}{\varepsilon-\varepsilon^{\alpha}}<-\frac{\varepsilon^{3-\alpha}}{\varepsilon}=-\varepsilon^{2-\alpha}$.

Applying a change of variables. using the definition of $u_{\varepsilon}$ and considering only one face of $\mathcal{T}_{\varepsilon}^{0}$, we get:

$$
\overline{\mathcal{G}_{\varepsilon}}\left(u_{\varepsilon}\right)=\frac{1}{2} \cdot \varepsilon^{-2 \beta} \int_{\mathcal{C}_{3 / 2} \backslash \mathcal{C}}|\nabla \varphi|^{2} \mathrm{~d} x+\frac{1}{8} \cdot \varepsilon^{(-\alpha / 2-\beta)(2 l-2)+3 \alpha} \int_{\mathcal{C}_{3 / 2} \backslash \mathcal{C}}|\varphi|^{2 l-2} \mathrm{~d} x-\frac{\delta}{4} \cdot \varepsilon^{2+\alpha-l(\alpha / 2+\beta)} .
$$

Since in the last equation the integrals are bounded, in order to prove that $\overline{\mathcal{G}_{\varepsilon}}$ is unbounded from below, we want to show that there exists $\beta>0$ such that:

$$
\left\{\begin{array}{l}
\varepsilon^{-2 \beta}<\varepsilon^{2+\alpha-\alpha \cdot \frac{l}{2}-\beta \cdot l} \\
\varepsilon^{\alpha \cdot(4-l)-2 \beta \cdot(l-1)}<\varepsilon^{2+\alpha-\alpha \cdot \frac{l}{2}-\beta \cdot l}
\end{array} \quad \Leftrightarrow 2-\alpha \cdot \frac{2-l}{2}<\beta \cdot(l-2)<\alpha \cdot \frac{6-l}{2}-2 .\right.
$$

But the last inequality is equivalent with choosing $\alpha \in\left(2, \frac{4}{l-2}\right)$, hence, $\overline{\mathcal{G}_{\varepsilon}}$ is unbounded from below and, since $\mathcal{G}_{\varepsilon}(\cdot) \leq \overline{\mathcal{G}_{\varepsilon}}(\cdot)$, we conclude our proof.

Remark 4.9. The previous lemma has been adapted from Lemma 3.6 from [13]. In a similar fashion, one can use Lemma 3.7 from [13] to prove similar results for the case in which $\alpha>\frac{4}{l-2}$.

\subsection{Lower semi-continuity of $\mathcal{F}_{\varepsilon}$}

Proposition 4.10. Suppose that the assumptions $\left(A_{1}\right)-\left(A_{7}\right)$ are satisfied. Then, the following statement holds: for any positive $M>0$, there exists $\varepsilon_{0}(M)>0$ such that for any $\varepsilon \in\left(0, \varepsilon_{0}(M)\right)$ and for any sequence $\left(Q_{j}\right)_{j \in \mathbb{N}}$ from $H^{1}\left(\Omega_{\varepsilon}, \mathcal{S}_{0}\right)$ that converges $H^{1}$-weakly to a function $Q \in H^{1}\left(\Omega_{\varepsilon}, \mathcal{S}_{0}\right)$ and which satisfies $\left\|\nabla Q_{j}\right\|_{L^{2}\left(\Omega_{\varepsilon}\right)} \leq M$ for any $j \in \mathbb{N}$, then

$$
\mathcal{F}_{\varepsilon}[Q] \leq \liminf _{j \rightarrow+\infty} \mathcal{F}_{\varepsilon}\left[Q_{j}\right]
$$

Proof. The proof of Proposition 4.10 follows the same steps as in [13]. We prove this proposition on each component of $\mathcal{F}_{\varepsilon}$. Before that, let

$$
\omega_{0}=\liminf _{j \rightarrow+\infty} \int_{\Omega_{\varepsilon}}\left|\nabla Q_{j}\right|^{2} \mathrm{~d} x-\int_{\Omega_{\varepsilon}}|\nabla Q|^{2} \mathrm{~d} x .
$$

Since $Q_{j} \rightarrow Q$ in $H^{1}$, then $\nabla Q_{j} \rightarrow \nabla Q$ in $L^{2}$, therefore $\omega_{0} \geq 0$. Moreover, up to extracting a subsequence, we can assume that

$$
\int_{\Omega_{\varepsilon}}\left|\nabla Q_{j}\right|^{2} \mathrm{~d} x \rightarrow \int_{\Omega_{\varepsilon}}|\nabla Q|^{2} \mathrm{~d} x+\omega_{0}
$$

as $j \rightarrow+\infty$.

From the assumption $\left(A_{5}\right)$, we have that $f_{e}$ is strongly convex, that is for $\theta>0$ small enough, $\tilde{f}_{e}(D):=$ $f_{e}(D)-\theta|D|^{2}$ is a convex function from $\mathcal{S}_{0} \otimes \mathbb{R}^{3}$ to $[0,+\infty)$. In this case, the functional $\int_{\Omega_{\varepsilon}} \tilde{f}_{e}(\cdot) \mathrm{d} x$ is lower semicontinuous. Therefore

$$
\liminf _{j \rightarrow+\infty} \int_{\Omega_{\varepsilon}} \tilde{f}_{e}\left(\nabla Q_{j}\right) \mathrm{d} x \geq \int_{\Omega_{\varepsilon}} \tilde{f}_{e}(\nabla Q) \mathrm{d} x
$$


from which we get

$$
\liminf _{j \rightarrow+\infty} \int_{\Omega_{\varepsilon}} f_{e}\left(\nabla Q_{j}\right) \mathrm{d} x-\int_{\Omega_{\varepsilon}} f_{e}(\nabla Q) \mathrm{d} x \geq\left(\liminf _{j \rightarrow+\infty} \int_{\Omega_{\varepsilon}} \tilde{f}_{e}\left(\nabla Q_{j}\right) \mathrm{d} x-\int_{\Omega_{\varepsilon}} \tilde{f}_{e}(\nabla Q) \mathrm{d} x\right)+\theta \omega_{0} \geq 0 .
$$

Since $Q_{j} \rightarrow Q$ in $H^{1}\left(\Omega_{\varepsilon}\right)$ and the injection $H^{1}\left(\Omega_{\varepsilon}\right) \subset L^{2}\left(\Omega_{\varepsilon}\right)$ is compact, then we can assume, up to extracting a subsequence, that $Q_{j} \rightarrow Q$ a.e. in $\Omega_{\varepsilon}$. Then, from the assumption $\left(A_{6}\right)$, we can see that the sequence $\left(f_{b}\left(Q_{j}\right)\right)_{j \in \mathbb{N}}$ satisfies all the conditions from Fatou's lemma, therefore:

$$
\liminf _{j \rightarrow+\infty} \int_{\Omega_{\varepsilon}} f_{b}\left(Q_{j}\right) \mathrm{d} x \geq \int_{\Omega_{\varepsilon}} \liminf _{j \rightarrow+\infty} f_{b}\left(Q_{j}\right) \mathrm{d} x=\int_{\Omega_{\varepsilon}} f_{b}(Q) \mathrm{d} x
$$

Regarding the surface energy, we split $\partial \mathcal{N}_{\varepsilon}^{\mathcal{T}}$ into:

$$
\begin{aligned}
A_{j} & =\left\{x \in \partial \mathcal{N}_{\varepsilon}^{\mathcal{T}}:\left|Q_{j}(x)-Q(x)\right| \leq|Q(x)|+1\right\} \\
B_{j}=\partial \mathcal{N}_{\varepsilon}^{\mathcal{T}} \backslash A_{j} & =\left\{x \in \partial \mathcal{N}_{\varepsilon}^{\mathcal{T}}:\left|Q_{j}(x)-Q(x)\right|>|Q(x)|+1\right\},
\end{aligned}
$$

for any $j \in \mathbb{N}$.

Using $\left(A_{7}\right)$, we have

$$
\begin{aligned}
\int_{A_{j}}\left|f_{s}\left(Q_{j}, \nu\right)-f_{s}(Q, \nu)\right| \mathrm{d} \sigma & \leq \int_{A_{j}}\left(\left|Q_{j}\right|^{3}+|Q|^{3}+1\right) \cdot\left|Q_{j}-Q\right| \mathrm{d} \sigma \\
& \leq \int_{A_{j}}\left(\left(\left|Q_{j}-Q\right|+|Q|\right)^{3}+|Q|^{3}+1\right) \cdot(|Q|+1) \mathrm{d} \sigma \\
& \lesssim \int_{A_{j}}\left(|Q|^{3}+1\right)(|Q|+1) \mathrm{d} \sigma \lesssim \int_{A_{j}}\left(|Q|^{4}+1\right) \mathrm{d} \sigma .
\end{aligned}
$$

Then due to the continuous embedding of $H^{1 / 2}\left(\partial \mathcal{N}_{\varepsilon}\right)$ into $L^{4}\left(\partial \mathcal{N}_{\varepsilon}\right)$ :

$$
\frac{\varepsilon^{3-\alpha}}{\varepsilon-\varepsilon^{\alpha}} \int_{A_{j}}\left|f_{s}\left(Q_{j}, \nu\right)-f_{s}(Q, \nu)\right| \mathrm{d} \sigma \lesssim \frac{\varepsilon^{3-\alpha}}{\varepsilon-\varepsilon^{\alpha}} \int_{A_{j}}\left(|Q|^{4}+1\right) \mathrm{d} \sigma<+\infty,
$$

according also to Proposition A.3. At the same time, the compact embedding $H^{1 / 2}\left(\partial \mathcal{N}_{\varepsilon}\right) \hookrightarrow L^{2}\left(\partial \mathcal{N}_{\varepsilon}\right)$ and the continuity of the trace operator from $H^{1}\left(\Omega_{\varepsilon}\right)$ into $H^{1 / 2}\left(\partial \mathcal{N}_{\varepsilon}\right)$ grants that $Q_{j} \rightarrow Q$ a.e. on $\partial \mathcal{N}_{\varepsilon}$, up to extracting a subsequence. We can now apply the dominated convergence theorem and get:

$$
\frac{\varepsilon^{3-\alpha}}{\varepsilon-\varepsilon^{\alpha}} \int_{A_{j}}\left|f_{s}\left(Q_{j}, \nu\right)-f_{s}(Q, \nu)\right| \mathrm{d} \sigma \rightarrow 0 \text { as } j \rightarrow+\infty .
$$

Regarding the $B_{j}$ sets, we have, according to $\left(A_{7}\right)$ :

$$
\begin{aligned}
\left|f_{s}\left(Q_{j}, \nu\right)-f_{s}(Q, \nu)\right| & \leq \lambda_{s}\left|Q_{j}-Q\right|\left(\left|Q_{j}\right|^{3}+|Q|^{3}+1\right) \\
& \lesssim\left|Q_{j}-Q\right|\left(\left|Q_{j}\right|^{3}+\left|Q_{j}-Q\right|^{3}+1\right)\left(\text { using }|Q|+1<\left|Q_{j}-Q\right|\right) \\
& \lesssim\left|Q_{j}-Q\right|\left(\left|Q_{j}-Q\right|^{3}+|Q|^{3}+\left|Q_{j}-Q\right|^{3}+1\right) \\
& \lesssim\left|Q_{j}-Q\right|\left(\left|Q_{j}-Q\right|^{3}+1\right) \lesssim\left|Q_{j}-Q\right|^{4} .
\end{aligned}
$$


Using Lemma 4.3 for $\left(Q_{j}-Q\right)$ with $n=4$, we have:

$$
\begin{aligned}
& \frac{\varepsilon^{3-\alpha}}{\varepsilon-\varepsilon^{\alpha}} \int_{B_{j}}\left|f_{s}\left(Q_{j}, \nu\right)-f_{s}(Q, \nu)\right| \mathrm{d} \sigma \lesssim \frac{\varepsilon^{3-\alpha}}{\varepsilon-\varepsilon^{\alpha}} \int_{B_{j}}\left|Q_{j}-Q\right|^{4} \mathrm{~d} \sigma \lesssim \frac{\varepsilon^{3-\alpha}}{\varepsilon-\varepsilon^{\alpha}} \int_{\partial \mathcal{N}_{\varepsilon}^{\mathcal{T}}}\left|Q_{j}-Q\right|^{4} \mathrm{~d} \sigma \\
& \quad \lesssim \frac{2 \varepsilon^{2-\alpha}}{1-\varepsilon^{\alpha-1}} \int_{\Omega_{\varepsilon}}\left|Q_{j}-Q\right|^{6}+\left|\nabla Q_{j}-\nabla Q\right|^{2} \mathrm{~d} x+\frac{1}{2\left(1-\varepsilon^{\alpha-1}\right)^{2}} \int_{\Omega_{\varepsilon}}\left|Q_{j}-Q\right|^{4} \mathrm{~d} x .
\end{aligned}
$$

Since $\mathrm{H}^{1}\left(\Omega_{\varepsilon}\right)$ is compactly embedded into $\mathrm{L}^{4}\left(\Omega_{\varepsilon}\right)$ and $Q_{j} \rightarrow Q$ in $\mathrm{H}^{1}\left(\Omega_{\varepsilon}\right)$, then $Q_{j} \rightarrow Q$ in $\mathrm{L}^{4}\left(\Omega_{\varepsilon}\right)$ and so

$$
\frac{1}{2\left(1-\varepsilon^{\alpha-1}\right)^{2}} \int_{\Omega_{\varepsilon}}\left|Q_{j}-Q\right|^{4} \mathrm{~d} x \rightarrow 0, \text { as } j \rightarrow+\infty .
$$

For the term containing $\left|Q_{j}-Q\right|^{6}$, we proceed in the following way:

$$
\begin{aligned}
\int_{\Omega_{\varepsilon}}\left|Q_{j}-Q\right|^{6} \mathrm{~d} x & =\int_{\Omega_{\varepsilon}}\left|E_{\varepsilon}\left(Q_{j}-Q\right)\right|^{6} \mathrm{~d} x \leq \int_{\Omega}\left|E_{\varepsilon}\left(Q_{j}-Q\right)\right|^{6} \mathrm{~d} x=\left\|E_{\varepsilon}\left(Q_{j}-Q\right)\right\|_{L^{6}(\Omega)}^{6} \\
& \lesssim\left\|E_{\varepsilon}\left(Q_{j}-Q\right)\right\|_{\mathrm{H}^{1}(\Omega)}^{6}\left(\text { by the continuous injection } \mathrm{H}^{1}(\Omega) \subset \mathrm{L}^{6}(\Omega)\right) \\
& \lesssim\left\|E_{\varepsilon}\left(Q_{j}-Q\right)\right\|_{\mathrm{H}_{0}^{1}(\Omega)}^{6}\left(\text { because } Q_{j} \equiv Q \text { on } \partial \Omega\right) \\
& \lesssim\left\|\nabla E_{\varepsilon}\left(Q_{j}-Q\right)\right\|_{\mathrm{L}^{2}(\Omega)}^{6}=\left(\int_{\Omega}\left|\nabla E_{\varepsilon}\left(Q_{j}-Q\right)\right|^{2} \mathrm{~d} x\right)^{3} \\
& \lesssim\left(\int_{\Omega_{\varepsilon}}\left|\nabla Q_{j}-\nabla Q\right|^{2} \mathrm{~d} x\right)^{3} \text { (using Lem. 4.4). }
\end{aligned}
$$

Now, because $\left\|\nabla Q_{j}\right\|_{L^{2}\left(\Omega_{\varepsilon}\right)} \leq M$, then:

$$
\int_{\Omega_{\varepsilon}}\left|\nabla Q_{j}-\nabla Q\right|^{2} \mathrm{~d} x \leq \int_{\Omega_{\varepsilon}}\left(\left|\nabla Q_{j}\right|^{2}+|\nabla Q|^{2}\right) \mathrm{d} x \lesssim M^{2}
$$

and therefore

$$
\frac{\varepsilon^{3-\alpha}}{\varepsilon-\varepsilon^{\alpha}} \int_{B_{j}}\left|f_{s}\left(Q_{j}, \nu\right)-f_{s}(Q, \nu)\right| \mathrm{d} \sigma \lesssim \frac{2 \varepsilon^{2-\alpha}}{1-\varepsilon^{\alpha-1}}\left(1+M^{4}\right) \int_{\Omega_{\varepsilon}}\left|\nabla Q_{j}-\nabla Q\right|^{2} \mathrm{~d} x+o(1) .
$$

Using that $Q_{j} \rightarrow Q$ in $\mathrm{H}^{1}\left(\Omega_{\varepsilon}\right)$ and (4.17), we obtain that $\int_{\Omega_{\varepsilon}}\left|\nabla Q_{j}-\nabla Q\right|^{2} \mathrm{~d} x \rightarrow \omega_{0}$ as $j \rightarrow+\infty$ and combining this with (4.20) and (4.21), we get:

$$
\liminf _{j \rightarrow+\infty} J_{\varepsilon}^{\mathcal{T}}\left[Q_{j}\right]-J_{\varepsilon}^{\mathcal{T}}[Q] \geq-C_{M} \cdot \omega_{0} \cdot \frac{\varepsilon^{2-\alpha}}{1-\varepsilon^{\alpha-1}},
$$

where $C_{M}$ is a constant dependent of $M$ and independent of $\varepsilon$.

According to (4.18), (4.19) and (4.22), we finally obtain that

$$
\liminf _{j \rightarrow+\infty} \mathcal{F}_{\varepsilon}\left[Q_{j}\right]-\mathcal{F}_{\varepsilon}[Q] \geq\left(\theta-C_{M} \frac{\varepsilon^{2-\alpha}}{1-\varepsilon^{\alpha-1}}\right) \omega_{0}
$$


and since $\frac{\varepsilon^{2-\alpha}}{1-\varepsilon^{\alpha-1}} \rightarrow 0$ as $\varepsilon \rightarrow 0$, the conclusion follows.

\section{CONVERGENCE OF LOCAL Minimisers}

\subsection{Pointwise convergence of the surface integral}

The aim of this section is to prove the following statement:

Theorem 5.1. Suppose that the assumptions $\left(A_{1}\right)-\left(A_{7}\right)$ are satisfied. Then, for any bounded, Lipschitz map $Q: \bar{\Omega} \rightarrow \mathcal{S}_{0}$, there holds $J_{\varepsilon}^{\mathcal{T}}[Q] \rightarrow J_{0}[Q]$ as $\varepsilon \rightarrow 0$, where

$$
J_{0}[Q]=\int_{\Omega} f_{h o m}(Q) \mathrm{d} x
$$

Proof. Let us fix a bounded, Lipschitz map $Q: \bar{\Omega} \rightarrow \mathcal{S}_{0}$ and let $\tilde{J}_{\varepsilon}$ be the following functional:

$$
\tilde{J}_{\varepsilon}[Q]=\frac{\varepsilon^{3-\alpha}}{\varepsilon-\varepsilon^{\alpha}}\left(\sum_{k=1}^{X_{\varepsilon}} \int_{\mathcal{T}_{x}^{k}} f_{s}\left(Q\left(y_{\varepsilon}^{x, k}\right), \nu\right) \mathrm{d} \sigma+\sum_{l=1}^{Y_{\varepsilon}} \int_{\mathcal{T}_{y}^{l}} f_{s}\left(Q\left(y_{\varepsilon}^{y, l}\right), \nu\right) \mathrm{d} \sigma+\sum_{m=1}^{Z_{\varepsilon}} \int_{\mathcal{T}_{z}^{m}} f_{s}\left(Q\left(y_{\varepsilon}^{z, m}\right), \nu\right) \mathrm{d} \sigma\right),
$$

where $y_{\varepsilon}^{x, k}, y_{\varepsilon}^{y, l}$ and $y_{\varepsilon}^{z, m}$ are defined in (A.3), (A.8) and (A.13), $\mathcal{T}_{x}^{k}, \mathcal{T}_{y}^{l}$ and $\mathcal{T}_{z}^{m}$ are defined in (A.6), (A.11) and (A.16) and $X_{\varepsilon}, Y_{\varepsilon}$ and $Z_{\varepsilon}$ are defined in (A.1), (A.7) and (A.12).

We prove that $\tilde{J}_{\varepsilon}[Q] \rightarrow J_{0}[Q]$ and that $\left|J_{\varepsilon}^{\mathcal{T}}[Q]-\tilde{J}_{\varepsilon}[Q]\right| \rightarrow 0$ as $\varepsilon \rightarrow 0$, for any $Q$ with the properties set earlier.

Let

$$
\left\{\begin{array}{l}
\Psi^{X}\left(Q\left(\tau_{0}\right)\right)=\int_{\mathcal{C}^{x}} f_{s}\left(Q\left(\tau_{0}\right), \nu(\tau)\right) \mathrm{d} \sigma(\tau) \\
\Psi^{Y}\left(Q\left(\tau_{0}\right)\right)=\int_{\mathcal{C}^{y}} f_{s}\left(Q\left(\tau_{0}\right), \nu(\tau)\right) \mathrm{d} \sigma(\tau) \\
\Psi^{Z}\left(Q\left(\tau_{0}\right)\right)=\int_{\mathcal{C}^{z}} f_{s}\left(Q\left(\tau_{0}\right), \nu(\tau)\right) \mathrm{d} \sigma(\tau)
\end{array}\right.
$$

for any $\tau_{0} \in \Omega$, where $\mathcal{C}^{x}, \mathcal{C}^{y}$ and $\mathcal{C}^{z}$ are defined in (2.3). Because $f_{s}$ is continuous on $\mathcal{S}_{0} \times \mathbb{S}^{2}$, then $\Psi^{X}, \Psi^{Y}$ and $\Psi^{Z}$ are also continuous. In this case, for example, the first sum from (5.2), denoted as $\tilde{J}_{\varepsilon}^{X}$, becomes:

$$
\begin{aligned}
\tilde{J}_{\varepsilon}^{X}[Q] & =\frac{\varepsilon^{3-\alpha}}{\varepsilon-\varepsilon^{\alpha}} \sum_{k=1}^{X_{\varepsilon}} \int_{\mathcal{T}_{x}^{k}} f_{s}\left(Q\left(y_{\varepsilon}^{x, k}\right), \nu\right) \mathrm{d} \sigma \\
& =\frac{\left(p-\varepsilon^{\alpha-1}\right)}{p r\left(1-\varepsilon^{\alpha-1}\right)} \cdot \varepsilon^{3} \sum_{k=1}^{X_{\varepsilon}} \int_{\mathcal{C}^{y}} f_{s}\left(Q\left(y_{\varepsilon}^{x, k}\right), \nu\right) \mathrm{d} \sigma+\frac{\left(p-\varepsilon^{\alpha-1}\right)}{p q\left(1-\varepsilon^{\alpha-1}\right)} \cdot \varepsilon^{3} \sum_{k=1}^{X_{\varepsilon}} \int_{\mathcal{C}^{z}} f_{s}\left(Q\left(y_{\varepsilon}^{x, k}\right), \nu\right) \mathrm{d} \sigma \\
& =\frac{\left(p-\varepsilon^{\alpha-1}\right)}{p r\left(1-\varepsilon^{\alpha-1}\right)} \cdot \int_{\Omega} \Psi^{Y}(Q(\tau)) \mathrm{d} \mu_{\varepsilon}^{X}(\tau)+\frac{\left(p-\varepsilon^{\alpha-1}\right)}{p q\left(1-\varepsilon^{\alpha-1}\right)} \cdot \int_{\Omega} \Psi^{Z}(Q(\tau)) \mathrm{d} \mu_{\varepsilon}^{X}(\tau),
\end{aligned}
$$

where $\mu_{\varepsilon}^{X}$ is defined in (2.9), that is, assumption $\left(A_{4}\right)$.

According to $\left(A_{4}\right)$, as $\varepsilon \rightarrow 0, \mu_{\varepsilon}^{X}$ converges weakly* to the Lebesgue measure restricted to $\Omega$ and because $\Psi^{Y}$ and $\Psi^{Z}$ are continuous, then:

$$
\tilde{J}_{\varepsilon}^{X}[Q] \rightarrow \frac{1}{r} \int_{\Omega} \Psi^{Y}(Q(\tau)) \mathrm{d} \tau+\frac{1}{q} \int_{\Omega} \Psi^{Z}(Q(\tau)) \mathrm{d} \tau .
$$


Computing in a similar way $\tilde{J}_{\varepsilon}^{y}$ and $\tilde{J}_{\varepsilon}^{z}$, we get:

$$
\tilde{J}_{\varepsilon}[Q] \rightarrow \int_{\Omega}\left(\frac{q+r}{q r} \Psi^{X}(Q(\tau))+\frac{p+r}{p r} \Psi^{Y}(Q(\tau))+\frac{p+q}{p q} \Psi^{Z}(Q(\tau))\right) \mathrm{d} \tau=\int_{\Omega} f_{h o m}(Q(\tau)) \mathrm{d} \tau=J_{0}[Q]
$$

which implies that $\tilde{J}_{\varepsilon}[Q] \rightarrow J_{0}[Q]$. For $J_{\varepsilon}^{X}[Q]$ and $\tilde{J}_{\varepsilon}^{X}[Q]$, we have:

$$
\begin{aligned}
\left|J_{\varepsilon}^{X}[Q]-\tilde{J}_{\varepsilon}^{X}[Q]\right| & \leq \frac{\varepsilon^{3-\alpha}}{\varepsilon-\varepsilon^{\alpha}} \sum_{k=1}^{X_{\varepsilon}} \int_{\mathcal{T}_{x}^{k}}\left|f_{s}(Q(\tau), \nu(\tau))-f_{s}\left(Q\left(y_{\varepsilon}^{x, k}\right), \nu(\tau)\right)\right| \mathrm{d} \sigma(\tau) \\
& \lesssim \frac{\varepsilon^{3-\alpha}}{\varepsilon-\varepsilon^{\alpha}} \sum_{k=1}^{X_{\varepsilon}} \int_{\mathcal{T}_{x}^{k}}\left(|Q(\tau)|^{3}+\left|Q\left(y_{\varepsilon}^{x, k}\right)\right|^{3}+1\right)\left|Q(\tau)-Q\left(y_{\varepsilon}^{x, k}\right)\right| \mathrm{d} \sigma(\tau) \\
& \lesssim \frac{\varepsilon^{3-\alpha}}{\varepsilon-\varepsilon^{\alpha}} \cdot\left(\|Q\|_{L^{\infty}(\bar{\Omega})}^{3}+1\right) \cdot \operatorname{Lip}(Q) \cdot \sum_{k=1}^{X_{\varepsilon}} \sigma\left(\mathcal{T}_{x}^{k}\right) \cdot \operatorname{diam}\left(\mathcal{T}_{x}^{k}\right),
\end{aligned}
$$

using that $Q$ is bounded on $\bar{\Omega}$ and where $\operatorname{Lip}(Q)$ is the Lipschitz constant of $Q, \sigma\left(\mathcal{T}_{x}^{k}\right)$ is the total area of $\mathcal{T}_{x}^{k}$ and $\operatorname{diam}\left(\mathcal{T}_{x}^{k}\right)$ is the diameter of $\mathcal{T}_{x}^{k}$, which coincides with the diameter of the parallelepiped $\mathcal{P}_{\varepsilon}^{x, k}$, defined in (A.4). Hence

$$
\left|J_{\varepsilon}^{X}[Q]-\tilde{J}_{\varepsilon}^{X}[Q]\right| \lesssim \frac{2(r+q)}{p q r} \cdot \frac{p \varepsilon-\varepsilon^{\alpha}}{\varepsilon-\varepsilon^{\alpha}} \cdot X_{\varepsilon} \cdot \varepsilon^{3} \cdot \sqrt{\left(\varepsilon-\frac{\varepsilon^{\alpha}}{p}\right)^{2}+\left(\frac{\varepsilon^{\alpha}}{q}\right)^{2}+\left(\frac{\varepsilon^{\alpha}}{r}\right)^{2}}
$$

Now, as $\varepsilon \rightarrow 0$, we have: $\sqrt{\left(\varepsilon-\frac{\varepsilon^{\alpha}}{p}\right)^{2}+\left(\frac{\varepsilon^{\alpha}}{q}\right)^{2}+\left(\frac{\varepsilon^{\alpha}}{r}\right)^{2}} \rightarrow 0 ; \frac{p \varepsilon-\varepsilon^{\alpha}}{\varepsilon-\varepsilon^{\alpha}}=\frac{p-\varepsilon^{\alpha-1}}{1-\varepsilon^{1-\alpha}} \rightarrow p$ because $1<\alpha$; $X_{\varepsilon} \cdot \varepsilon^{3}<\left(\frac{L_{0}}{\varepsilon}-1\right) \cdot \frac{l_{0}}{\varepsilon} \cdot \frac{h_{0}}{\varepsilon} \cdot \varepsilon^{3}=L_{0} l_{0} h_{0}-\varepsilon l_{0} h_{0}$, according to Proposition A.2, where $L_{0}, l_{0}$ and $h_{0}$ are defined in (2.1). Since $X_{\varepsilon}$ is positive, we see that:

$$
0 \leq \lim _{\varepsilon \rightarrow 0}\left(X_{\varepsilon} \cdot \varepsilon^{3}\right) \leq \lim _{\varepsilon \rightarrow 0}\left(L_{0} l_{0} h_{0}-\varepsilon l_{0} h_{0}\right)=L_{0} l_{0} h_{0}<+\infty
$$

Therefore $J_{\varepsilon}^{X}[Q] \rightarrow \tilde{J}_{\varepsilon}^{X}[Q]$ as $\varepsilon \rightarrow 0$. We get the same result for the other two components, from which we conclude.

Remark 5.2. It is easy to see that if we replace the coefficient $\frac{\varepsilon^{3}}{\varepsilon^{\alpha}\left(\varepsilon-\varepsilon^{\alpha}\right)}$ of the surface energy term $J_{\varepsilon}$ with:

$$
\begin{aligned}
& -\frac{\varepsilon^{3}}{\left(\varepsilon-\varepsilon^{\alpha}\right)^{2}} \rightarrow 0, \text { then } J_{\varepsilon}^{X}[Q] \rightarrow 0 \text { as } \varepsilon \rightarrow 0 ; \\
& -\frac{\varepsilon^{3}}{\varepsilon^{2 \alpha}}=\varepsilon^{3-2 \alpha} \rightarrow 0, \text { then } J_{\varepsilon}^{X}[Q] \rightarrow+\infty \text { as } \varepsilon \rightarrow 0 .
\end{aligned}
$$

In both cases, we lose the convergence $J_{\varepsilon}^{\mathcal{T}}[Q] \rightarrow J_{0}[Q]$. 


\section{2. $\Gamma$-convergence of the approximating free energies}

Lemma 5.3. Suppose that the assumption $\left(A_{7}\right)$ is satisfied. Let $Q_{1}$ and $Q_{2}$ from $H_{g}^{1}\left(\Omega, \mathcal{S}_{0}\right)$ be such that

$$
\max \left\{\left\|\nabla Q_{1}\right\|_{L^{2}(\Omega)},\left\|\nabla Q_{2}\right\|_{L^{2}(\Omega)}\right\} \leq M
$$

for some $\varepsilon$-independent constant $M$. Then, for $\varepsilon$ sufficiently small, we have:

$$
\left|J_{\varepsilon}^{\mathcal{T}}\left[Q_{2}\right]-J_{\varepsilon}^{\mathcal{T}}\left[Q_{1}\right]\right| \leq C_{M}\left(\varepsilon^{1 / 2-\alpha / 4}+\left\|Q_{2}-Q_{1}\right\|_{L^{4}(\Omega)}\right)
$$

for some $C_{M}>0$ depending only on $M, f_{s}, \Omega, \mathcal{C}$ and $g$.

Proof. According to $\left(A_{7}\right)$ and Hölder inequality, we have:

$$
\begin{gathered}
\left|J_{\varepsilon}^{\mathcal{T}}\left[Q_{2}\right]-J_{\varepsilon}^{\mathcal{T}}\left[Q_{1}\right]\right| \lesssim \frac{\varepsilon^{3-\alpha}}{\varepsilon-\varepsilon^{\alpha}} \int_{\partial \mathcal{N}_{\varepsilon}^{\mathcal{T}}}\left(\left|Q_{1}\right|^{3}+\left|Q_{2}\right|^{3}+1\right)\left|Q_{2}-Q_{1}\right| \mathrm{d} \sigma \\
\lesssim \frac{\varepsilon^{3-\alpha}}{\varepsilon-\varepsilon^{\alpha}}\left(\int_{\partial \mathcal{N}_{\varepsilon}^{\mathcal{T}}}\left|Q_{2}-Q_{1}\right|^{4} \mathrm{~d} \sigma\right)^{1 / 4}\left(\left(\int_{\partial \mathcal{N}_{\varepsilon}^{\mathcal{T}}}\left|Q_{1}\right|^{4} \mathrm{~d} \sigma\right)^{3 / 4}+\left(\int_{\partial \mathcal{N}_{\varepsilon}^{\mathcal{T}}}\left|Q_{2}\right|^{4} \mathrm{~d} \sigma\right)^{3 / 4}+\left|\partial \mathcal{N}_{\varepsilon}^{\mathcal{T}}\right|^{3 / 4}\right) .
\end{gathered}
$$

If we make use of Lemma 4.3, then:

$$
\frac{\varepsilon^{3-\alpha}}{\varepsilon-\varepsilon^{\alpha}} \int_{\partial \mathcal{N}_{\varepsilon}^{\mathcal{T}}}\left|Q_{i}\right|^{4} \mathrm{~d} \sigma \lesssim \frac{2 \varepsilon^{2-\alpha}}{1-\varepsilon^{\alpha-1}} \int_{\Omega_{\varepsilon}}\left(\left|Q_{i}\right|^{6}+\left|\nabla Q_{i}\right|^{2}\right) \mathrm{d} x+\frac{1}{2\left(1-\varepsilon^{\alpha-1}\right)^{2}} \int_{\Omega_{\varepsilon}}\left|Q_{i}\right|^{4} \mathrm{~d} x
$$

for any $i \in\{1,2\}$. By the continuous injection $\mathrm{H}^{1}\left(\Omega_{\varepsilon}\right)$ into $\mathrm{L}^{6}\left(\Omega_{\varepsilon}\right)$, we have

$$
\frac{\varepsilon^{3-\alpha}}{\varepsilon-\varepsilon^{\alpha}} \int_{\partial \mathcal{N}_{\varepsilon}^{\mathcal{T}}}\left|Q_{i}\right|^{4} \mathrm{~d} \sigma \lesssim \frac{2 \varepsilon^{2-\alpha}}{1-\varepsilon^{\alpha-1}}\left(\left\|\nabla Q_{i}\right\|_{\mathrm{L}^{2}\left(\Omega_{\varepsilon}\right)}^{2}+\left\|Q_{i}\right\|_{\mathrm{H}^{1}\left(\Omega_{\varepsilon}\right)}^{6}\right)+\frac{1}{2\left(1-\varepsilon^{\alpha-1}\right)^{2}}\left\|Q_{i}\right\|_{\mathrm{L}^{4}\left(\Omega_{\varepsilon}\right)}^{4} .
$$

Using the Poincaré inequality as in Theorem 4.4.7, page 193, from [26], the compact embedding $H^{1}\left(\Omega_{\varepsilon}\right) \hookrightarrow L^{4}\left(\Omega_{\varepsilon}\right)$ and the fact that $\Omega_{\varepsilon} \subset \Omega$, we get for $\varepsilon$ small enough:

$$
\begin{aligned}
\frac{\varepsilon^{3-\alpha}}{\varepsilon-\varepsilon^{\alpha}} \int_{\partial \mathcal{N}_{\varepsilon}^{\mathcal{T}}}\left|Q_{i}\right|^{4} \mathrm{~d} \sigma & \lesssim \frac{2 \varepsilon^{2-\alpha}}{1-\varepsilon^{\alpha-1}}\left(\left\|\nabla Q_{i}\right\|_{L^{2}(\Omega)}^{2}+\left\|\nabla Q_{i}\right\|_{L^{2}(\Omega)}^{6}\right)+\frac{1}{2\left(1-\varepsilon^{\alpha-1}\right)^{2}}\left\|\nabla Q_{i}\right\|_{L^{2}(\Omega)}^{4} \\
& \lesssim \frac{2 \varepsilon^{2-\alpha}}{1-\varepsilon^{\alpha-1}}\left(M^{2}+M^{6}\right)+\frac{1}{2\left(1-\varepsilon^{\alpha-1}\right)^{2}} M^{4}
\end{aligned}
$$

where we used (5.8) and we can see that the right-hand side from the last inequality can be bounded in terms of $M$, since $\frac{\varepsilon^{2-\alpha}}{1-\varepsilon^{\alpha-1}} \searrow 0$ and $\frac{1}{\left(1-\varepsilon^{\alpha-1}\right)^{2}} \searrow 1$ as $\varepsilon \rightarrow 0$. But since $\frac{1}{\left(1-\varepsilon^{\alpha-1}\right)^{2}} \searrow 1$ as $\varepsilon \rightarrow 0$, we can choose $\varepsilon>0$ such that $\frac{1}{\left(1-\varepsilon^{\alpha-1}\right)^{2}}<2$ and we can move the constant 2 under the "¿" sign. Hence, the last relation can be written as:

$$
\frac{\varepsilon^{3-\alpha}}{\varepsilon-\varepsilon^{\alpha}} \int_{\partial \mathcal{N}_{\varepsilon}^{\mathcal{T}}}\left|Q_{i}\right|^{4} \mathrm{~d} \sigma \lesssim \varepsilon^{2-\alpha}\left(M^{2}+M^{6}\right)+M^{4}
$$


In a similar fashion, using the same arguments as before for (5.10), we get in the case of $\left(Q_{2}-Q_{1}\right)$ :

$$
\frac{\varepsilon^{3-\alpha}}{\varepsilon-\varepsilon^{\alpha}} \int_{\partial \mathcal{N}_{\varepsilon}^{\mathcal{T}}}\left|Q_{2}-Q_{1}\right|^{4} \mathrm{~d} \sigma \lesssim \varepsilon^{2-\alpha}\left(M^{2}+M^{6}\right)+\left\|Q_{2}-Q_{1}\right\|_{L^{4}(\Omega)}^{4}
$$

Using the same bounds as in (5.10), we conclude by observing that there exists a constant $C_{M}>0$ such that:

$$
\left|J_{\varepsilon}^{\mathcal{T}}\left[Q_{2}\right]-J_{\varepsilon}^{\mathcal{T}}\left[Q_{1}\right]\right| \leq C_{M} \cdot\left(\left(\varepsilon^{2-\alpha}\right)^{1 / 4}+\left\|Q_{2}-Q_{1}\right\|_{L^{4}(\Omega)}\right)
$$

Lemma 5.4. For any $Q \in H_{g}^{1}\left(\Omega, \mathcal{S}_{0}\right)$, there holds $J_{\varepsilon}^{\mathcal{T}}[Q] \rightarrow J_{0}[Q]$ as $\varepsilon \rightarrow 0$.

Proof. Let $\left(Q_{j}\right)_{j \geq 1}$ be a sequence of smooth functions that converge strongly to $Q$ in $H_{g}^{1}\left(\Omega, \mathcal{S}_{0}\right)$. Then there holds:

$$
\left|J_{\varepsilon}^{\mathcal{T}}[Q]-J_{0}[Q]\right| \leq\left|J_{\varepsilon}^{\mathcal{T}}[Q]-J_{\varepsilon}^{\mathcal{T}}\left[Q_{j}\right]\right|+\left|J_{\varepsilon}^{\mathcal{T}}\left[Q_{j}\right]-J_{0}\left[Q_{j}\right]\right|+\left|J_{0}\left[Q_{j}\right]-J_{0}[Q]\right|
$$

From Lemma 5.3, we have that

$$
\left|J_{\varepsilon}^{\mathcal{T}}[Q]-J_{\varepsilon}^{\mathcal{T}}\left[Q_{j}\right]\right| \lesssim \varepsilon^{1 / 2-\alpha / 4}+\left\|Q-Q_{j}\right\|_{L^{4}(\Omega)}
$$

and we recall that for $\varepsilon \rightarrow 0$ we have $\varepsilon^{1 / 2-\alpha / 4} \rightarrow 0$ because $\alpha \in(1,2)$.

Since the $\left(Q_{j}\right)_{j \geq 1}$ converge strongly in $H_{g}^{1}(\Omega)$, from the compact Sobolev embedding, we get that $Q_{j} \rightarrow Q$ in $L^{4}(\Omega)$ as $j \rightarrow+\infty$, therefore $Q_{j} \rightarrow Q$ a.e. in $\Omega$.

From Theorem 5.1, we obtain that $J_{\varepsilon}^{\mathcal{T}}\left[Q_{j}\right] \rightarrow J_{0}\left[Q_{j}\right]$ as $\varepsilon \rightarrow 0$, for any $j \geq 1$.

For the last term, we can write $\left|J_{0}\left[Q_{j}\right]-J_{0}[Q]\right| \leq \int_{\Omega}\left|f_{\text {hom }}\left[Q_{j}\right]-f_{\text {hom }}[Q]\right| \mathrm{d} x$. In here, we have: $f_{\text {hom }}$ is continuous, $Q_{j} \rightarrow Q$ a.e. in $\Omega$ and $f_{\text {hom }}$ has a quartic growth in $Q$ (because $f_{s}$ has the same growth), which implies that: $\left|f_{\text {hom }}\left[Q_{j}\right]\right| \lesssim\left|Q_{j}\right|^{4}+1$. At the same time, we can assume that there exists $\psi \in L^{1}(\Omega)$ such that $\left|Q_{j}\right|^{4} \leq \psi$, for any $j \geq 1$, a.e. in $\Omega$. Therefore, we can apply the Lebesgue dominated convergence theorem and get that $J_{0}\left[Q_{j}\right] \rightarrow J_{0}[Q]$ as $j \rightarrow+\infty$.

Combining the results from above, we obtain:

$$
\limsup _{\varepsilon \rightarrow 0}\left|J_{\varepsilon}^{\mathcal{T}}[Q]-J_{\varepsilon}^{\mathcal{T}}\left[Q_{j}\right]\right| \lesssim\left\|Q-Q_{j}\right\|_{L^{4}(\Omega)}+\left|J_{0}\left[Q_{j}\right]-J_{0}[Q]\right| \rightarrow 0, \quad \text { as } j \rightarrow+\infty,
$$

from which we conclude.

We now prove that $\mathcal{F}_{\varepsilon} \Gamma$-converges to $\mathcal{F}_{0}$ as $\varepsilon \rightarrow 0$, with respect to the weak $H^{1}$-topology.

Proposition 5.5. Suppose that the assumptions $\left(A_{1}\right)-\left(A_{7}\right)$ are satisfied. Let $Q_{\varepsilon} \in H_{g}^{1}\left(\Omega_{\varepsilon}, \mathcal{S}_{0}\right)$ be such that $E_{\varepsilon} Q_{\varepsilon} \rightarrow Q$ weakly in $H^{1}(\Omega)$ as $\varepsilon \rightarrow 0$. Then:

$$
\liminf _{\varepsilon \rightarrow 0} \mathcal{F}_{\varepsilon}\left[Q_{\varepsilon}\right] \geq \mathcal{F}_{0}[Q], \quad \lim _{\varepsilon \rightarrow 0} J_{\varepsilon}^{\mathcal{T}}\left[Q_{\varepsilon}\right]=J_{0}[Q] .
$$

Proof. The proof follows the same steps as in Proposition 4.2. from [13].

Since $E_{\varepsilon} Q_{\varepsilon} \rightarrow Q$ in $H^{1}(\Omega)$, then $\left(E_{\varepsilon} Q_{\varepsilon}\right)_{\varepsilon>0}$ is a bounded sequence in $H^{1}(\Omega)$. Therefore, we can choose a subsequence $\left(E_{\varepsilon_{j}} Q_{\varepsilon_{j}}\right)_{j \geq 1} \subset\left(E_{\varepsilon} Q_{\varepsilon}\right)_{\varepsilon>0}$ such that

$$
\liminf _{\varepsilon \rightarrow 0} \mathcal{F}_{\varepsilon}\left[Q_{\varepsilon}\right]=\lim _{j \rightarrow+\infty} \mathcal{F}_{\varepsilon_{j}}\left[Q_{\varepsilon_{j}}\right]
$$


Furthermore, by the compact embeddings $H^{1}(\Omega) \hookrightarrow L^{s}(\Omega)$, with $s \in[1,6)$, we have that $E_{\varepsilon_{j}} Q_{\varepsilon_{j}} \rightarrow Q$ strongly in $L^{s}(\Omega)$, for any $s \in[1,6)$. As a result, we also obtain that $E_{\varepsilon_{j}} Q_{\varepsilon_{j}} \rightarrow Q$ a.e. in $\Omega$. We denote the subsequence $E_{\varepsilon_{j}} Q_{\varepsilon_{j}}$ as $E_{\varepsilon} Q_{\varepsilon}$ for the ease of notation.

Now, according to $\left(A_{5}\right)$, we have:

$$
\begin{aligned}
\int_{\Omega_{\varepsilon}}\left(f_{e}\left(\nabla Q_{\varepsilon}\right)-f_{e}(\nabla Q)\right) \mathrm{d} x & \geq \int_{\Omega_{\varepsilon}} \nabla f_{e}(\nabla Q):\left(\nabla Q_{\varepsilon}-\nabla Q\right) \mathrm{d} x \\
& =\int_{\Omega} \nabla f_{e}(\nabla Q):\left(\nabla Q_{\varepsilon}-\nabla Q\right) \mathrm{d} x-\int_{\mathcal{N}_{\varepsilon}} \nabla f_{e}(\nabla Q):\left(\nabla Q_{\varepsilon}-\nabla Q\right) \mathrm{d} x \\
& \geq \int_{\Omega} \nabla f_{e}(\nabla Q):\left(\nabla Q_{\varepsilon}-\nabla Q\right) \mathrm{d} x-\left\|\nabla f_{e}(\nabla Q)\right\|_{L^{2}\left(\mathcal{N}_{\varepsilon}\right)} \cdot\left\|\nabla Q_{\varepsilon}-\nabla Q\right\|_{L^{2}\left(\mathcal{N}_{\varepsilon}\right)}
\end{aligned}
$$

Because $Q \in H^{1}(\Omega)$, then $\nabla Q \in L^{2}(\Omega)$ and, according to $\left(A_{5}\right)$, the relation $\left|\nabla f_{e}(\nabla Q)\right| \lesssim|\nabla Q|+1$ implies that $\nabla f_{e}(\nabla Q) \in L^{2}(\Omega)$. Therefore, by the weak convergence $E_{\varepsilon} Q_{\varepsilon} \rightarrow Q$ in $H^{1}(\Omega)$, the first term from the right hand side in (5.11) goes to 0 as $\varepsilon \rightarrow 0$. The second term goes to 0 as well thanks additionally to the fact that the volume of the scaffold $\mathcal{N}_{\varepsilon}$ tends to 0 as $\varepsilon \rightarrow 0$, according to Proposition A.2. Hence:

$$
\liminf _{\varepsilon \rightarrow 0} \int_{\Omega_{\varepsilon}} f_{e}\left(\nabla Q_{\varepsilon}\right) \mathrm{d} x \geq \lim _{\varepsilon \rightarrow 0} \int_{\Omega_{\varepsilon}} f_{e}(\nabla Q) \mathrm{d} x=\int_{\Omega} f_{e}(\nabla Q) \mathrm{d} x
$$

For the bulk potential we apply Fatou's lemma, since $f_{b}\left(Q_{\varepsilon}\right) \chi_{\Omega_{\varepsilon}} \rightarrow f_{b}(Q)$ a.e. in $\Omega$ (because $f_{b}$ is continuous, $E_{\varepsilon} Q_{\varepsilon} \rightarrow Q$ a.e. in $\Omega$ and $\left|\mathcal{N}_{\varepsilon}\right| \rightarrow 0$, according to Prop. A.2) and $f_{b}$ is bounded from below (according to $\left(A_{6}\right)$ ), in order to obtain:

$$
\liminf _{\varepsilon \rightarrow 0} \int_{\Omega_{\varepsilon}} f_{b}\left(Q_{\varepsilon}\right) \mathrm{d} x \geq \int_{\Omega} f_{b}(Q) \mathrm{d} x
$$

For the surface energy, we first use Lemma 5.3 in the following inequality:

$$
\begin{aligned}
\left|J_{\varepsilon}^{\mathcal{T}}\left[E_{\varepsilon} Q_{\varepsilon}\right]-J_{0}[Q]\right| & \leq\left|J_{\varepsilon}^{\mathcal{T}}\left[E_{\varepsilon} Q_{\varepsilon}\right]-J_{\varepsilon}^{\mathcal{T}}[Q]\right|+\left|J_{\varepsilon}^{\mathcal{T}}[Q]-J_{0}[Q]\right| \\
& \lesssim \varepsilon^{1 / 2-\alpha / 4}+\left\|E_{\varepsilon} Q_{\varepsilon}-Q\right\|_{L^{4}(\Omega)}+\left|J_{\varepsilon}^{\mathcal{T}}[Q]-J_{0}[Q]\right|
\end{aligned}
$$

Since we have $\varepsilon^{1 / 2-\alpha / 4} \rightarrow 0$ for $\varepsilon \rightarrow 0$ (because $\alpha \in(1,2)$ ), then combining the result from Lemma 5.4 with the fact that $E_{\varepsilon} Q_{\varepsilon} \rightarrow Q$ strongly in $L^{4}(\Omega)$, we obtain

$$
\lim _{\varepsilon \rightarrow 0} J_{\varepsilon}^{\mathcal{T}}\left[Q_{\varepsilon}\right]=J_{0}[Q] .
$$

The proof is now complete, considering (5.12), (5.13) and (5.14).

Proposition 5.6. Suppose that the assumptions $\left(A_{1}\right)-\left(A_{7}\right)$ are verified. Then, for any $Q \in H_{g}^{1}\left(\Omega, \mathcal{S}_{0}\right)$, there exists a sequence $\left(Q_{\varepsilon}\right)_{\varepsilon>0}$ such that $Q_{\varepsilon} \in H^{1}\left(\Omega_{\varepsilon}\right)$, for any $\varepsilon>0, E_{\varepsilon} Q_{\varepsilon} \rightarrow Q$ in $H^{1}(\Omega)$ and:

$$
\limsup _{\varepsilon \rightarrow 0} \mathcal{F}_{\varepsilon}\left[Q_{\varepsilon}\right] \leq \mathcal{F}_{0}[Q]
$$

The sequence $\left(Q_{\varepsilon}\right)_{\varepsilon>0}$ is called a recovery sequence. 
Proof. Let us define in this case $Q_{\varepsilon}=Q \cdot \chi_{\Omega_{\varepsilon}}$. Since $\left|\mathcal{N}_{\varepsilon}\right| \rightarrow 0$ as $\varepsilon \rightarrow 0$ (according to Prop. A.2), then $\chi_{\Omega_{\varepsilon}} \rightarrow 1$ strongly in $L^{1}(\Omega)$ and we can apply Lebesgue's dominated converge theorem in order to obtain that:

$$
\begin{aligned}
\lim _{\varepsilon \rightarrow 0} \int_{\Omega_{\varepsilon}} f_{e}\left(\nabla Q_{\varepsilon}\right)+f_{b}\left(Q_{\varepsilon}\right) \mathrm{d} x & =\int_{\Omega} f_{e}(\nabla Q)+f_{b}(Q) \mathrm{d} x \\
\lim _{\varepsilon \rightarrow 0}\left(\mathcal{F}_{\varepsilon}[Q]-J_{\varepsilon}^{\mathcal{T}}[Q]\right) & =\mathcal{F}_{0}[Q]-J_{0}[Q] .
\end{aligned}
$$

By Proposition 5.5, we have that $\lim _{\varepsilon \rightarrow 0} J_{\varepsilon}^{\mathcal{T}}\left[Q_{\varepsilon}\right]=J_{0}[Q]$, hence the conclusion follows.

Proposition 5.5 and Proposition 5.6 show that $\mathcal{F}_{\varepsilon} \Gamma$-converges to $\mathcal{F}_{0}$, as $\varepsilon \rightarrow 0$, with respect to the weak $H^{1}$ topology.

\subsection{Proof of main theorems}

Proof of Theorem 3.2. Let $Q_{0}$ from $H_{g}^{1}\left(\Omega, \mathcal{S}_{0}\right)$ be an isolated $H^{1}$-local minimiser for $\mathcal{F}_{0}$, that is, there exists $\delta_{0}>0$ such that $\mathcal{F}_{0}\left[Q_{0}\right]<\mathcal{F}_{0}[Q]$, for any $Q \in H_{g}^{1}\left(\Omega, \mathcal{S}_{0}\right)$, such that $0<\left\|Q-Q_{0}\right\|_{H^{1}(\Omega)} \leq \delta_{0}$.

We would like to prove that for any $\varepsilon>0$, there exists $Q_{\varepsilon} \in H_{g}^{1}\left(\Omega_{\varepsilon}, \mathcal{S}_{0}\right)$, which is a $H^{1}$-local minimiser for $\mathcal{F}_{\varepsilon}$, such that $E_{\varepsilon} Q_{\varepsilon} \rightarrow Q_{0}$ strongly in $H_{g}^{1}\left(\Omega, \mathcal{S}_{0}\right)$ as $\varepsilon \rightarrow 0$.

For this, let

$$
\mathcal{B}_{\varepsilon}:=\left\{Q \in H_{g}^{1}\left(\Omega_{\varepsilon}, \mathcal{S}_{0}\right):\left\|E_{\varepsilon} Q-Q_{0}\right\|_{H^{1}(\Omega)} \leq \delta_{0}\right\}
$$

Using Mazur's lemma, we can show that the set $\mathcal{B}_{\varepsilon}$ is sequentially weakly closed in $H^{1}\left(\Omega_{\varepsilon}\right)$. Then, by Proposition 4.10, we can see that, for $\varepsilon$ small enough, $\mathcal{F}_{\varepsilon}$ is lower semicontinuous on $\mathcal{B}_{\varepsilon}$ and, by Proposition 4.7, is also coercive on $\mathcal{B}_{\varepsilon}$, since any $Q \in \mathcal{B}_{\varepsilon}$ has $\|\nabla Q\|_{L^{2}\left(\Omega_{\varepsilon}\right)}<\left\|\nabla Q_{0}\right\|_{L^{2}(\Omega)}+\delta_{0}$. Hence, for any $\varepsilon$ sufficiently small, the functional $\mathcal{F}_{\varepsilon}$ admits at least one minimiser $Q_{\varepsilon}$ from $\mathcal{B}_{\varepsilon}$.

Firstly, we prove that $E_{\varepsilon} Q_{\varepsilon} \rightarrow Q_{0}$ weakly in $H^{1}(\Omega)$, as $\varepsilon \rightarrow 0$.

Let $\mathcal{B}_{0}:=\left\{Q \in H_{g}^{1}\left(\Omega, \mathcal{S}_{0}\right):\left\|Q-Q_{0}\right\|_{H^{1}(\Omega)} \leq \delta_{0}\right\}$. Because $Q_{\varepsilon} \in \mathcal{B}_{\varepsilon}$, then $\left(E_{\varepsilon} Q_{\varepsilon}\right)_{\varepsilon>0}$ represents a bounded sequence in $H^{1}(\Omega)$, hence there exists a subsequence, which we still denote $\left(E_{\varepsilon} Q_{\varepsilon}\right)_{\varepsilon>0}$ for the ease of notation, that converges weakly to a $\tilde{Q} \in \mathcal{B}_{0}$. We show that $\tilde{Q}=Q_{0}$.

Since $E_{\varepsilon} Q_{\varepsilon} \rightarrow \tilde{Q}$ in $H_{g}^{1}\left(\Omega, \mathcal{S}_{0}\right)$, we can apply Proposition 5.5 and get:

$$
\mathcal{F}_{0}[\tilde{Q}] \leq \liminf _{\varepsilon \rightarrow 0} \mathcal{F}_{\varepsilon}\left[Q_{\varepsilon}\right] \leq \limsup _{\varepsilon \rightarrow 0} \mathcal{F}_{\varepsilon}\left[Q_{\varepsilon}\right]
$$

But $Q_{\varepsilon}$ is a minimiser of $\mathcal{F}_{\varepsilon}$ on $\mathcal{B}_{\varepsilon}$, therefore, since $\left.Q_{0}\right|_{\Omega_{\varepsilon}} \in \mathcal{B}_{\varepsilon}$, we get that

$$
\limsup _{\varepsilon \rightarrow 0} \mathcal{F}_{\varepsilon}\left[Q_{\varepsilon}\right] \leq \lim _{\varepsilon \rightarrow 0} \mathcal{F}_{\varepsilon}\left[Q_{0}\right]=\lim _{\varepsilon \rightarrow 0}\left(\int_{\Omega_{\varepsilon}} f_{e}\left(\nabla Q_{0}\right)+f_{b}(Q) \mathrm{d} x+\mathcal{J}_{\varepsilon}^{\mathcal{T}}\left[Q_{0}\right]\right)=\mathcal{F}_{0}\left[Q_{0}\right]
$$

Hence, we have $\mathcal{F}_{0}[\tilde{Q}] \leq \mathcal{F}_{0}\left[Q_{0}\right]$. Because $\tilde{Q}$ is in $\mathcal{B}_{0}$, that is $\left\|\tilde{Q}-Q_{0}\right\|_{H^{1}(\Omega)} \leq \delta_{0}$, then by the definition of $Q_{0}$, we get that $\tilde{Q}=Q_{0}$.

We now prove that $E_{\varepsilon} Q_{\varepsilon} \rightarrow Q_{0}$ strongly in $H^{1}(\Omega)$, as $\varepsilon \rightarrow 0$.

By $\left(A_{5}\right)$, there exists $\theta>0$ such that the function $\tilde{f}_{e}(D)=f_{e}(D)-\theta|D|^{2}$ is convex. We can repeat the same arguments from Proposition 5.5, more specifically, steps (5.12) and (5.13), to get:

$$
\liminf _{\varepsilon \rightarrow 0} \int_{\Omega_{\varepsilon}} \tilde{f}_{e}\left(\nabla Q_{\varepsilon}\right) \mathrm{d} x \geq \int_{\Omega} \tilde{f}_{e}\left(\nabla Q_{0}\right) \mathrm{d} x,
$$




$$
\begin{gathered}
\theta \liminf _{\varepsilon \rightarrow 0} \int_{\Omega_{\varepsilon}}\left|\nabla Q_{\varepsilon}\right|^{2} \mathrm{~d} x \geq \theta \int_{\Omega}\left|\nabla Q_{0}\right|^{2} \mathrm{~d} x, \\
\liminf _{\varepsilon \rightarrow 0} \int_{\Omega_{\varepsilon}} f_{b}\left(Q_{\varepsilon}\right) \mathrm{d} x \geq \int_{\Omega} f_{b}\left(Q_{0}\right) \mathrm{d} x .
\end{gathered}
$$

From Proposition 5.5, we have that $J_{\varepsilon}^{\mathcal{T}}\left[Q_{\varepsilon}\right] \rightarrow J_{0}\left[Q_{0}\right]$ as $\varepsilon \rightarrow 0$. Also, from the proof that $\tilde{Q}=Q_{0}$, we can see that

$$
\lim _{\varepsilon \rightarrow 0} \mathcal{F}_{\varepsilon}\left[Q_{\varepsilon}\right]=\mathcal{F}_{0}\left[Q_{0}\right]
$$

which implies that

$$
\lim _{\varepsilon \rightarrow 0} \int_{\Omega_{\varepsilon}}\left|\nabla Q_{\varepsilon}\right|^{2} \mathrm{~d} x=\int_{\Omega}\left|\nabla Q_{0}\right|^{2} \mathrm{~d} x
$$

This shows us that

$$
\nabla\left(E_{\varepsilon} Q_{\varepsilon}\right) \chi_{\Omega_{\varepsilon}} \rightarrow \nabla Q_{0} \text { strongly in } L^{2}(\Omega)
$$

where $\chi_{\Omega_{\varepsilon}}$ is the characteristic function of $\Omega_{\varepsilon}$.

We now show that $\nabla\left(E_{\varepsilon} Q_{\varepsilon}\right) \chi_{\mathcal{N}_{\varepsilon}}$ converges strongly to 0 in $L^{2}(\Omega)$. In order to prove this, in Appendix A.3, we obtain that:

$$
\left\|\nabla E_{\varepsilon} Q_{\varepsilon}\right\|_{L^{2}\left(\mathcal{P}_{\varepsilon}^{z, m}\right)} \leq C \cdot\left\|\nabla Q_{\varepsilon}\right\|_{L^{2}\left(\mathcal{R}_{\varepsilon}^{z, m}\right)},
$$

where $\mathcal{P}_{\varepsilon}^{z, m}$ is a "connecting parallelepiped" elongated in the $O z$ direction and $\mathcal{R}_{\varepsilon}^{z, m}$ is a 3D object that "surrounds" $\mathcal{P}_{\varepsilon}^{z, m}$. More specifically, we have that:

$$
\mathcal{P}_{\varepsilon}^{z, m}=y_{\varepsilon}^{z, m}+\left[-\frac{\varepsilon^{\alpha}}{2 p}, \frac{\varepsilon^{\alpha}}{2 p}\right] \times\left[-\frac{\varepsilon^{\alpha}}{2 q}, \frac{\varepsilon^{\alpha}}{2 q}\right] \times\left[-\frac{r \varepsilon-\varepsilon^{\alpha}}{2 r}, \frac{r \varepsilon-\varepsilon^{\alpha}}{2 r}\right]
$$

and

$\mathcal{R}_{\varepsilon}^{z, m}=y_{\varepsilon}^{z, m}+\left(\left(\left[-\frac{3 \varepsilon^{\alpha}}{4 p}, \frac{3 \varepsilon^{\alpha}}{4 p}\right] \times\left[-\frac{3 \varepsilon^{\alpha}}{4 q}, \frac{3 \varepsilon^{\alpha}}{4 q}\right]\right) \backslash\left(\left[-\frac{\varepsilon^{\alpha}}{2 p}, \frac{\varepsilon^{\alpha}}{2 p}\right] \times\left[-\frac{\varepsilon^{\alpha}}{2 q}, \frac{\varepsilon^{\alpha}}{2 q}\right]\right)\right) \times\left[-\frac{r \varepsilon-\varepsilon^{\alpha}}{2 r}, \frac{r \varepsilon-\varepsilon^{\alpha}}{2 r}\right]$,

where $y_{\varepsilon}^{z, m}$ is the center of $\mathcal{P}_{\varepsilon}^{z, m}$.

In this way, we have that $\left|\mathcal{R}_{\varepsilon}^{z, m}\right|=\frac{5}{4} \cdot\left|\mathcal{P}_{\varepsilon}^{z, m}\right|$ and $\mathcal{R}_{\varepsilon}^{z, m} \subset \Omega_{\varepsilon}$. Moreover, if we take two objects of the type $\mathcal{R}_{\varepsilon}^{z, m}$, then it is easy to see that they are disjoint: in the $O z$ direction, $\mathcal{R}_{\varepsilon}^{z, m}$ has the same height as $\mathcal{P}_{\varepsilon}^{z, m}$ and in the $x O y$ plane the "surrounding" objects are not touching because we consider $\varepsilon \rightarrow 0$ and, since $\alpha \in(1,2)$, we have $\varepsilon^{\alpha}<<\varepsilon$. Applying this technique from Appendix A.3 for all the "connecting parallelepipeds" of the type $\mathcal{R}_{\varepsilon}^{z, m}$, we obtain that there exists an $\varepsilon$-independent constant such that:

$$
\left\|\nabla E_{\varepsilon} Q_{\varepsilon}\right\|_{L^{2}\left(\cup \mathcal{P}_{\varepsilon}^{z, m}\right)} \leq C \cdot\left\|\nabla Q_{\varepsilon}\right\|_{L^{2}\left(\cup \mathcal{R}_{\varepsilon}^{z, m}\right)},
$$

and repeating in the same way for all the other "connecting parallelepipeds", we obtain:

$$
\left\|\nabla E_{\varepsilon} Q_{\varepsilon}\right\|_{L^{2}\left(\mathcal{N}_{\varepsilon}^{\mathcal{T}}\right)} \leq C \cdot\left\|\nabla Q_{\varepsilon}\right\|_{L^{2}\left(\mathcal{R}_{\varepsilon}^{\mathcal{T}}\right)},
$$


where $\mathcal{R}_{\varepsilon}^{\mathcal{T}}$ represents the union of all "surrounding" objects for the "connecting parallelepipeds".

For the "inner parallelepipeds", we can apply the same technique as before. If the "inner parallelepiped" is not "visible" - meaning that is has six adjacent "connecting parallelepipeds" - then the "surrounding" object constructed is included in the union of the six adjacent "connecting parallelipepds" and their respective "surounding" bodies. If the "inner parallelepiped" is close to the boundary - meaning that it has strictly less than 6 adjacent "connecting parallelepipeds" - then the "surrounding" object constructed has a part included in the adjacent "connecting parallelepipeds" and their respective "surrounding" bodies, but also a part which was not taken into account until now. But this "surrounding" object will have its volume only $5 / 4$ times bigger than the "inner parallelepiped" chosen and the same technique as for the "connecting parallelepipeds" can be applied. In this way, one can obtain the following inequality:

$$
\left\|\nabla E_{\varepsilon} Q_{\varepsilon}\right\|_{L^{2}\left(\mathcal{N}_{\varepsilon}^{\mathcal{S}}\right)} \leq C\left\|\nabla Q_{\varepsilon}\right\|_{L^{2}\left(\mathcal{R}_{\varepsilon}^{\mathcal{S}} \cup \mathcal{R}_{\varepsilon}^{\mathcal{T}}\right)},
$$

where the constant used is $\varepsilon$-independent and $\mathcal{R}_{\varepsilon}^{\mathcal{S}}$ is a $3 \mathrm{D}$ object with its volume tending to 0 as $\varepsilon \rightarrow 0$ and represents the union of all "surrounding" parts for the "inner parallelepipeds" that are not included in either the "connecting parallelepipeds" or their respective "surrounding" bodies.

Hence, we can obtain that:

$$
\left\|\nabla E_{\varepsilon} Q_{\varepsilon}\right\|_{L^{2}\left(\mathcal{N}_{\varepsilon}\right)} \leq C \cdot\left\|\nabla Q_{\varepsilon}\right\|_{L^{2}\left(\mathcal{R}_{\varepsilon}\right)},
$$

where $\mathcal{R}_{\varepsilon}=\mathcal{R}_{\varepsilon}^{\mathcal{T}} \cup \mathcal{R}_{\varepsilon}^{\mathcal{S}} \subset \Omega_{\varepsilon}$ with $\left|\mathcal{R}_{\varepsilon}\right| \rightarrow 0$ as $\varepsilon \rightarrow 0$.

Due to our definition of the extension operator $E_{\varepsilon}$, we have $E_{\varepsilon} Q_{\varepsilon} \equiv Q_{\varepsilon}$ in $\Omega_{\varepsilon}$, so we can write the last inequality as follows:

$$
\left\|\nabla E_{\varepsilon} Q_{\varepsilon}\right\|_{L^{2}\left(\mathcal{N}_{\varepsilon}\right)} \leq C \cdot\left\|\nabla E_{\varepsilon} Q_{\varepsilon}\right\|_{L^{2}\left(\mathcal{R}_{\varepsilon}\right)} .
$$

Now we want to prove that the right hand side term from the last inequality tends to 0 as $\varepsilon \rightarrow 0$. For this, we use:

$$
\begin{aligned}
\int_{\mathcal{R}_{\varepsilon}}\left|\nabla E_{\varepsilon} Q_{\varepsilon}\right|^{2} \mathrm{~d} x & =\int_{\Omega_{\varepsilon}}\left|\nabla E_{\varepsilon} Q_{\varepsilon}\right|^{2} \cdot \chi_{\mathcal{R}_{\varepsilon}} \mathrm{d} x \leq \int_{\Omega_{\varepsilon}} 2 \cdot\left(\left|\nabla E_{\varepsilon} Q_{\varepsilon}-\nabla Q_{0}\right|^{2}+\left|\nabla Q_{0}\right|^{2}\right) \cdot \chi_{\mathcal{R}_{\varepsilon}} \mathrm{d} x \\
& \leq 2 \int_{\Omega_{\varepsilon}}\left|\nabla E_{\varepsilon} Q_{\varepsilon}-\nabla Q_{0}\right|^{2} \mathrm{~d} x+2 \int_{\Omega}\left|\nabla Q_{0}\right|^{2} \cdot \chi_{\mathcal{R}_{\varepsilon}} \mathrm{d} x \\
& \leq 2 \int_{\Omega}\left|\left(\nabla E_{\varepsilon} Q_{\varepsilon}\right) \chi_{\Omega_{\varepsilon}}-\nabla Q_{0}\right|^{2} \mathrm{~d} x+2 \int_{\Omega}\left|\nabla Q_{0}\right|^{2} \cdot \chi_{\mathcal{R}_{\varepsilon}} \mathrm{d} x,
\end{aligned}
$$

where we have used that $\Omega_{\varepsilon} \subset \Omega$. Now the right hand side of the last inequality tends to 0 : the first integral converges to 0 due to the fact that $\left(\nabla E_{\varepsilon} Q_{\varepsilon}\right) \chi_{\Omega_{\varepsilon}} \rightarrow \nabla Q_{0}$ strongly in $L^{2}(\Omega)$, according to (5.15); the second integral converges to 0 because we can apply the dominated convergence theorem, since $\left|\nabla Q_{0}\right|^{2} \chi_{\mathcal{R}_{\varepsilon}}$ converges almost everywhere to 0 in $\Omega$ because $\left|\mathcal{R}_{\varepsilon}\right| \rightarrow 0$. Going back to (5.16), we obtain that $\left(\nabla E_{\varepsilon} Q_{\varepsilon}\right) \chi_{\mathcal{N}_{\varepsilon}} \rightarrow 0$ strongly in $L^{2}(\Omega)$, as $\varepsilon \rightarrow 0$.

Combining all the results, we obtain that $\nabla E_{\varepsilon} Q_{\varepsilon} \rightarrow \nabla Q_{0}$ strongly in $L^{2}(\Omega)$, hence $E_{\varepsilon} Q_{\varepsilon}$ converges strongly to $Q_{0}$ in $H^{1}(\Omega)$, since the weak convergence $E_{\varepsilon} Q_{\varepsilon} \rightarrow Q_{0}$ in $H^{1}(\Omega)$ automatically implies the strong convergence $E_{\varepsilon} Q_{\varepsilon} \rightarrow Q_{0}$ in $L^{2}(\Omega)$. 


\section{RAte of CONVERGENCE}

The aim of this section is the study the rate of convergence of the sequence $J_{\varepsilon}^{\mathcal{T}}\left[Q_{\varepsilon}\right]$ to $J_{0}\left[Q_{0}\right]$, where $J_{\varepsilon}^{\mathcal{T}}$ is defined in (4.10) and in (4.11), $J_{0}$ is defined in (5.1) and $\left(Q_{\varepsilon}\right)_{\varepsilon>0}$ is a sequence from $H_{g}^{1}\left(\Omega, \mathcal{S}_{0}\right)$ that converges $H^{1}$-strongly to $Q \in H_{g}^{1}\left(\Omega, \mathcal{S}_{0}\right)$. We omit the term $J_{\mathcal{E}}^{\mathcal{S}}$ because in Section 4.2 we proved that this term has no contribution to the homogenised functional.

First, we recall some notations used in the previous sections. For a $Q \in H_{g}^{1}\left(\Omega, \mathcal{S}_{0}\right)$, we write $J_{0}[Q]$ in the following form:

$$
\begin{aligned}
J_{0}[Q] & =\int_{\Omega}\left(\frac{1}{r} \Psi^{Y}(Q)+\frac{1}{q} \Psi^{Z}(Q)\right) \mathrm{d} x \\
& +\int_{\Omega}\left(\frac{1}{r} \Psi^{X}(Q)+\frac{1}{p} \Psi^{Z}(Q)\right) \mathrm{d} x \\
& +\int_{\Omega}\left(\frac{1}{q} \Psi^{X}(Q)+\frac{1}{p} \Psi^{Y}(Q)\right) \mathrm{d} x,
\end{aligned}
$$

where $\Psi^{X}, \Psi^{Y}$ and $\Psi^{Z}$ are defined in (5.3). We also write $\tilde{J}_{\varepsilon}[Q]$, defined in (5.2), as:

$$
\begin{aligned}
\tilde{J}_{\varepsilon}[Q] & =\int_{\Omega}\left(\frac{p \varepsilon-\varepsilon^{\alpha}}{p r\left(\varepsilon-\varepsilon^{\alpha}\right)} \Psi^{Y}(Q)+\frac{p \varepsilon-\varepsilon^{\alpha}}{p q\left(\varepsilon-\varepsilon^{\alpha}\right)} \Psi^{Z}(Q)\right) \mathrm{d} \mu_{\varepsilon}^{X} \\
& +\int_{\Omega}\left(\frac{q \varepsilon-\varepsilon^{\alpha}}{q r\left(\varepsilon-\varepsilon^{\alpha}\right)} \Psi^{X}(Q)+\frac{q \varepsilon-\varepsilon^{\alpha}}{p q\left(\varepsilon-\varepsilon^{\alpha}\right)} \Psi^{Z}(Q)\right) \mathrm{d} \mu_{\varepsilon}^{Y} \\
& +\int_{\Omega}\left(\frac{r \varepsilon-\varepsilon^{\alpha}}{q r\left(\varepsilon-\varepsilon^{\alpha}\right)} \Psi^{X}(Q)+\frac{r \varepsilon-\varepsilon^{\alpha}}{p r\left(\varepsilon-\varepsilon^{\alpha}\right)} \Psi^{Y}(Q)\right) \mathrm{d} \mu_{\varepsilon}^{Z},
\end{aligned}
$$

using (5.4) and the analogous formulae.

We suppose now that:

$\left(H_{1}\right)$ the surface energy density $f_{s}$ is locally Lipschitz continuous.

Using the assumption $\left(A_{7}\right)$, from Section 2, we have:

$$
\left|f_{s}\left(Q_{1}, \nu\right)-f_{s}\left(Q_{2}, \nu\right)\right| \lesssim\left|Q_{2}-Q_{1}\right|\left(\left|Q_{1}\right|^{3}+\left|Q_{2}\right|^{3}+1\right)
$$

for any $Q_{1}, Q_{2} \in \mathcal{S}_{0}$ and any $\nu \in \mathbb{S}^{2}$, and

$$
\left|f_{s}(Q, \nu)\right| \lesssim|Q|^{4}+1
$$

for any $Q \in \mathcal{S}_{0}$ and any $\nu \in \mathbb{S}^{2}$.

We now have the following lemma:

Lemma 6.1. For any $K \in\{X, Y, Z\}$, the function $\Psi^{K}$ is locally Lipschitz continuous and there holds:

$$
\left|\Psi^{K}(Q)\right| \lesssim|Q|^{4}+1 \quad\left|\nabla \Psi^{K}(Q)\right| \lesssim|Q|^{3}+1,
$$

for any $Q \in \mathcal{S}_{0}$. Moreover, the function $\Psi^{K}$ satisfies:

$$
\left|\Psi^{K}\left(Q_{1}\right)-\Psi^{K}\left(Q_{2}\right)\right| \lesssim\left|Q_{2}-Q_{1}\right|\left(\left|Q_{1}\right|^{3}+\left|Q_{2}\right|^{3}+1\right),
$$

for any $Q_{1}, Q_{2} \in \mathcal{S}_{0}$. 
Proof. The proof of this lemma follows immediatly, using the definitions of the functions $\Psi^{X}, \Psi^{Y}$ and $\Psi^{Z}$ from (5.3), the assumption $\left(H_{1}\right)$ and the properties of the function $f_{s}$ from (6.3) and (6.4).

We recall now that the measures $\mu_{\varepsilon}^{X}, \mu_{\varepsilon}^{Y}$ and $\mu_{\varepsilon}^{Z}$, which are defined in (2.9), converge weakly*, as measures in $\mathbb{R}^{3}$, to the Lebesgue measure restricted to $\Omega$, according to $\left(A_{4}\right)$ from Section 2 . We need to prescribe a rate of convergence and for this we use the $W^{-1,1}$-norm (that is, the dual Lipschitz norm, also known as flat norm in some contexts):

$$
\mathbb{F}_{\varepsilon}:=\max _{K \in\{X, Y, Z\}} \sup \left\{\int_{\Omega} \varphi \mathrm{d} \mu_{\varepsilon}^{K}-\int_{\Omega} \varphi \mathrm{d} x: \varphi \in W^{1, \infty}(\Omega),\|\nabla \varphi\|_{L^{\infty}(\Omega)}+\|\varphi\|_{L^{\infty}(\Omega)} \leq 1\right\} .
$$

Lemma 6.2. There exists a constant $\lambda_{\text {flat }}>0$ such that $\mathbb{F}_{\varepsilon} \leq \lambda_{\text {flat }} \varepsilon$ for any $\varepsilon>0$.

Proof. Let $\varphi \in W^{1, \infty}(\Omega)$. Then, according to the definition of $\mu_{\varepsilon}^{X}$ from (2.9), we have:

$$
\int_{\Omega} \varphi \mathrm{d} \mu_{\varepsilon}^{X}=\varepsilon^{3} \sum_{k=1}^{X_{\varepsilon}} \varphi\left(y_{\varepsilon}^{x, k}\right)=\sum_{k=1}^{X_{\varepsilon}} \int_{y_{\varepsilon}^{x, k}+[-\varepsilon / 2, \varepsilon / 2]^{3}} \varphi\left(y_{\varepsilon}^{x, k}\right) \mathrm{d} x,
$$

where in the last equality we integrate over the cube with length $\varepsilon$ centered in $y_{\varepsilon}^{x, k}$. Let $\Omega_{\varepsilon}^{X}$ be the following set:

$$
\Omega_{\varepsilon}^{X}:=\bigcup_{k=1}^{X_{\varepsilon}}\left(y_{\varepsilon}^{x, k}+[-\varepsilon / 2, \varepsilon / 2]^{3}\right) .
$$

Hence, we can write:

$$
\int_{\Omega} \varphi \mathrm{d} \mu_{\varepsilon}^{X}=\int_{\Omega_{\varepsilon}^{X}} \varphi\left(y_{\varepsilon}^{x, k}\right) \mathrm{d} x
$$

Then:

$$
\begin{aligned}
\left|\int_{\Omega} \varphi \mathrm{d} \mu_{\varepsilon}^{X}-\int_{\Omega} \varphi \mathrm{d} x\right| & \leq \int_{\Omega_{\varepsilon}^{X}}\left|\varphi-\varphi\left(y_{\varepsilon}^{x, k}\right)\right| \mathrm{d} x+\int_{\Omega \backslash \Omega_{\varepsilon}^{X}}|\varphi| \mathrm{d} x \\
& \leq \frac{\varepsilon \sqrt{3}}{2}\|\nabla \varphi\|_{L^{\infty}(\Omega)} \cdot\left|\Omega_{\varepsilon}^{X}\right|+\|\varphi\|_{L^{\infty}(\Omega)} \cdot\left|\Omega \backslash \Omega_{\varepsilon}^{X}\right|,
\end{aligned}
$$

where $\frac{\varepsilon \sqrt{3}}{2}$ comes from the largest possible value for $\left|x-y_{\varepsilon}^{x, k}\right|$, with $x \in\left(y_{\varepsilon}^{x, k}+[-\varepsilon / 2, \varepsilon / 2]^{3}\right)$.

If we look now at the definition of the points $y_{\varepsilon}^{x, k}$ in (A.2), hence also at the definition of the points $x_{\varepsilon}^{i}$ in (2.5) and (2.6), we observe that $\Omega \backslash \Omega_{\varepsilon}^{X} \subset\{x \in \Omega: \operatorname{dist}(x, \partial \Omega)<\varepsilon\}$, therefore, we have $\left|\Omega \backslash \Omega_{\varepsilon}^{X}\right| \leq C \cdot \varepsilon$, where $C$ is an $\varepsilon$-independent constant. At the same time, we have $\Omega_{\varepsilon}^{X} \subset \Omega \Rightarrow\left|\Omega_{\varepsilon}^{X}\right| \leq|\Omega|$, so (6.5) becomes:

$$
\left|\int_{\Omega} \varphi \mathrm{d} \mu_{\varepsilon}^{X}-\int_{\Omega} \varphi \mathrm{d} x\right| \leq \frac{\varepsilon \sqrt{3}}{2}\|\nabla \varphi\|_{L^{\infty}(\Omega)} \cdot|\Omega|+C \cdot \varepsilon\|\varphi\|_{L^{\infty}(\Omega)} \lesssim \varepsilon \cdot\|\varphi\|_{W^{1, \infty}(\Omega)} .
$$

Computing in the same fashion for $\mu_{\varepsilon}^{Y}$ and $\mu_{\varepsilon}^{Z}$, we obtain the conclusion.

We also suppose that: 
$\left(H_{2}\right) g$ is bounded and Lipschitz, where $g$ represents the prescribed boundary data.

Since $\Omega$ is bounded and smooth (by assumption $\left(A_{1}\right)$ from Sect. 2), we can extend the function $g$ to a bounded and Lipschitz map from $\mathbb{R}^{3}$ to $\mathcal{S}_{0}$, denoted still as $g$.

We present an auxiliary result proved in [14]:

Lemma 6.3. Let $\Omega \subseteq \mathbb{R}^{3}$ a bounded, smooth domain, and let $g: \Omega \rightarrow \mathcal{S}_{0}$ be a bounded, Lipschitz map. For any $Q \in H_{g}^{1}\left(\Omega, \mathcal{S}_{0}\right)$ and $\sigma \in(0,1)$, there exists a bounded, Lipschitz map $Q_{\sigma}: \bar{\Omega} \rightarrow \mathcal{S}_{0}$ that satisfies the following properties:

$$
\begin{gathered}
Q_{\sigma}=g \quad \text { on } \partial \Omega \\
\left\|Q_{\sigma}\right\|_{L^{\infty}(\Omega)} \lesssim \sigma^{-1 / 2}\left(\|Q\|_{H^{1}(\Omega)}+\|g\|_{L^{\infty}(\Omega)}\right) \\
\left\|\nabla Q_{\sigma}\right\|_{L^{\infty}(\Omega)} \lesssim \sigma^{-3 / 2}\left(\|Q\|_{H^{1}(\Omega)}+\|g\|_{W^{1, \infty}(\Omega)}\right) \\
\left\|Q-Q_{\sigma}\right\|_{L^{2}(\Omega)} \lesssim \sigma\|Q\|_{H^{1}(\Omega)} \\
\left\|\nabla Q-\nabla Q_{\sigma}\right\|_{L^{2}(\Omega)} \rightarrow 0 \quad \text { as } \sigma \rightarrow 0 .
\end{gathered}
$$

The main result from this section is the following:

Proposition 6.4. Suppose that assumptions $\left(A_{1}\right)-\left(A_{7}\right)$ (from Sect. 2) and $\left(H_{1}\right)-\left(H_{2}\right)$ (from this section) hold. Then, for any $Q \in H_{g}^{1}\left(\Omega, \mathcal{S}_{0}\right)$, there exists a sequence $\left(Q_{\varepsilon}\right)_{\varepsilon>0}$ in $H_{g}^{1}\left(\Omega, \mathcal{S}_{0}\right)$ that converges $H^{1}(\Omega)$-strongly to $Q$ and satisfies

$$
\left|J_{\varepsilon}^{\mathcal{T}}\left[Q_{\varepsilon}\right]-J_{0}[Q]\right| \lesssim \varepsilon^{m_{0}}\left(\|Q\|_{H^{1}(\Omega)}^{4}+1\right)
$$

for $\varepsilon$ small enough, where $J_{\varepsilon}^{\mathcal{T}}$ is defined in (4.10), $J_{0}$ is defined in (6.1) and $m_{0}=\min \left\{\frac{\alpha-1}{3}, 2-\alpha\right\}$. The $\varepsilon$-independent constant that is hidden by the use of the sign “ $\lesssim$ ", described as in Remark 2.5, depends only on the $L^{\infty}$-norms of $g$ and $\nabla g$, on $\Omega, f_{s}$ and the initial cube $\mathcal{C}$.

Remark 6.5. The previous proposition allows us to obtain, as claimed, a rate of convergence for the minimisers $\bar{Q}_{\varepsilon}$ of $\mathcal{F}_{\varepsilon}$ to a minimiser $Q$ of $\mathcal{F}_{0}$ in terms of $\left\|\bar{Q}_{\varepsilon}-Q\right\|_{H^{1}(\Omega)}=o(1)$ as $\varepsilon \rightarrow 0$ (i.e. relation (6.10)).

Indeed, this is obtained in the following way. First, let us fix a value for $0<\varepsilon<1$ such that equation (5.9) holds. Then we use the inequality

$$
\left|J_{\varepsilon}^{\mathcal{T}}\left[\bar{Q}_{\varepsilon}\right]-J_{0}[Q]\right| \leq\left|J_{\varepsilon}^{\mathcal{T}}\left[\bar{Q}_{\varepsilon}\right]-J_{\varepsilon}^{\mathcal{T}}\left[Q_{\varepsilon}\right]\right|+\left|J_{\varepsilon}^{\mathcal{T}}\left[Q_{\varepsilon}\right]-J_{0}[Q]\right|,
$$

where $Q_{\varepsilon}$ is the function from $H_{g}^{1}\left(\Omega, \mathcal{S}_{0}\right)$ granted by Lemma 6.3 , with $\sigma=\varepsilon^{m_{0}}$.

For the first term from the right-hand side from the last inequality, we use relation (5.9) and we obtain, for a fixed $\varepsilon$ sufficiently small:

$$
\left|J_{\varepsilon}^{\mathcal{T}}\left[\bar{Q}_{\varepsilon}\right]-J_{\varepsilon}^{\mathcal{T}}\left[Q_{\varepsilon}\right]\right| \leq C \cdot\left(\varepsilon^{1 / 2-\alpha / 4}+\left\|\bar{Q}_{\varepsilon}-Q_{\varepsilon}\right\|_{L^{4}(\Omega)}\right)
$$

where $C$ is $\varepsilon$-independent. 
From the compact Sobolev embedding $H^{1}(\Omega) \hookrightarrow L^{4}(\Omega)$, we obtain:

$$
\left|J_{\varepsilon}^{\mathcal{T}}\left[\bar{Q}_{\varepsilon}\right]-J_{\varepsilon}^{\mathcal{T}}\left[Q_{\varepsilon}\right]\right| \leq C \cdot\left(\varepsilon^{1 / 2-\alpha / 4}+\left\|\bar{Q}_{\varepsilon}-Q_{\varepsilon}\right\|_{H^{1}(\Omega)}\right) .
$$

Now, we observe that:

$$
\begin{aligned}
\left\|\bar{Q}_{\varepsilon}-Q_{\varepsilon}\right\|_{H^{1}(\Omega)} & \leq\left\|\bar{Q}_{\varepsilon}-Q\right\|_{H^{1}(\Omega)}+\left\|Q_{\varepsilon}-Q\right\|_{H^{1}(\Omega)} \\
& \leq\left\|\bar{Q}_{\varepsilon}-Q\right\|_{H^{1}(\Omega)}+\left\|Q_{\varepsilon}-Q\right\|_{L^{2}(\Omega)}+\left\|\nabla Q_{\varepsilon}-\nabla Q\right\|_{L^{2}(\Omega)} \\
& \leq\left\|\bar{Q}_{\varepsilon}-Q\right\|_{H^{1}(\Omega)}+\varepsilon^{m_{0}}\|Q\|_{H^{1}(\Omega)}+\left\|\nabla Q_{\varepsilon}-\nabla Q\right\|_{L^{2}(\Omega)},
\end{aligned}
$$

where we have used relation (6.8) in the last row. Relation (6.9) tells us that $\left\|\nabla Q_{\varepsilon}-\nabla Q\right\|_{L^{2}(\Omega)} \rightarrow 0$ as $\varepsilon \rightarrow 0$, hence, by the choice of $\varepsilon$, we can control it with a constant. Since $Q$ is fixed, we can also control $\|Q\|_{H^{1}(\Omega)}$ with an $\varepsilon$-independent constant. Therefore, we can write:

$$
\left\|\bar{Q}_{\varepsilon}-Q_{\varepsilon}\right\|_{H^{1}(\Omega)} \lesssim\left\|\bar{Q}_{\varepsilon}-Q\right\|_{H^{1}(\Omega)}+\varepsilon^{m_{0}} .
$$

Hence, we have:

$$
\left|J_{\varepsilon}^{\mathcal{T}}\left[\bar{Q}_{\varepsilon}\right]-J_{\varepsilon}^{\mathcal{T}}\left[Q_{\varepsilon}\right]\right| \lesssim \varepsilon^{1 / 2-\alpha / 4}+\varepsilon^{m_{0}}+\left\|\bar{Q}_{\varepsilon}-Q\right\|_{H^{1}(\Omega)} .
$$

and if we denote by $m_{\alpha}=\min \left\{1 / 2-\alpha / 4, m_{0}\right\}$ (which is defined depending whether $\alpha$ is bigger or smaller than $10 / 7)$, we can rewrite:

$$
\left|J_{\varepsilon}^{\mathcal{T}}\left[\bar{Q}_{\varepsilon}\right]-J_{\varepsilon}^{\mathcal{T}}\left[Q_{\varepsilon}\right]\right| \lesssim \varepsilon^{m_{\alpha}}+\left\|\bar{Q}_{\varepsilon}-Q\right\|_{H^{1}(\Omega)},
$$

since $\varepsilon$ is chosen from $(0,1)$.

For the term $\left|J_{\varepsilon}^{\mathcal{T}}\left[Q_{\varepsilon}\right]-J_{0}[Q]\right|$, we apply Proposition 6.4:

$$
\left|J_{\varepsilon}^{\mathcal{T}}\left[Q_{\varepsilon}\right]-J_{0}[Q]\right| \lesssim \varepsilon^{m_{0}}\left(\|Q\|_{H^{1}(\Omega)}^{4}+1\right)
$$

and since $Q$ is fixed, we obtain:

$$
\left|J_{\varepsilon}^{\mathcal{T}}\left[Q_{\varepsilon}\right]-J_{0}[Q]\right| \lesssim \varepsilon^{m_{0}} \lesssim \varepsilon^{m_{\alpha}},
$$

using the definition of $m_{\alpha}$.

If we go back to our initial inequality, we obtain:

$$
\left|J_{\varepsilon}^{\mathcal{T}}\left[\bar{Q}_{\varepsilon}\right]-J_{0}[Q]\right| \lesssim \varepsilon^{m_{\alpha}}+\left\|\bar{Q}_{\varepsilon}-Q\right\|_{H^{1}(\Omega)} .
$$

where

$$
m_{\alpha}=\left\{\begin{array}{l}
\frac{\alpha-1}{3}, 1<\alpha \leq \frac{10}{7} \\
\frac{2-\alpha}{4}, \frac{10}{7}<\alpha<2
\end{array}\right.
$$

Proof of Proposition 6.4. Let us fix a small $\varepsilon \in(0,1)$ such that:

$$
\frac{p \varepsilon-\varepsilon^{\alpha}}{\varepsilon-\varepsilon^{\alpha}}<2 p, \frac{q \varepsilon-\varepsilon^{\alpha}}{\varepsilon-\varepsilon^{\alpha}}<2 q \text { and } \frac{r \varepsilon-\varepsilon^{\alpha}}{\varepsilon-\varepsilon^{\alpha}}<2 r .
$$


This is possible since $\frac{p \varepsilon-\varepsilon^{\alpha}}{\varepsilon-\varepsilon^{\alpha}} \searrow p, \frac{q \varepsilon-\varepsilon^{\alpha}}{\varepsilon-\varepsilon^{\alpha}} \searrow q$ and $\frac{r \varepsilon-\varepsilon^{\alpha}}{\varepsilon-\varepsilon^{\alpha}} \searrow r$ as $\varepsilon \rightarrow 0$ and $p, q, r \geq 1$.

Let now $\beta$ be a positive parameter, to be chosen later, and let $Q_{\varepsilon}:=Q_{\varepsilon^{\beta}} \in H_{g}^{1}\left(\Omega, \mathcal{S}_{0}\right)$ be the Lipschitz map given by Lemma 6.3. Then, we have:

$$
\left|J_{\varepsilon}^{\mathcal{T}}\left[Q_{\varepsilon}\right]-J_{0}[Q]\right| \leq\left|J_{\varepsilon}^{\mathcal{T}}\left[Q_{\varepsilon}\right]-\tilde{J}_{\varepsilon}\left[Q_{\varepsilon}\right]\right|+\left|\tilde{J}_{\varepsilon}\left[Q_{\varepsilon}\right]-J_{0}\left[Q_{\varepsilon}\right]\right|+\left|J_{0}\left[Q_{\varepsilon}\right]-J_{0}[Q]\right|,
$$

where $\tilde{J}_{\varepsilon}$ is defined in $(5.2)$.

We analyse the first term from the right-hand side from (6.12). Using the same notations as in Theorem 5.1, replacing $\operatorname{Lip}\left(Q_{\varepsilon}\right)$ (the Lipschitz constant) with $\left\|\nabla Q_{\varepsilon}\right\|_{L^{\infty}(\Omega)}$ and combining relations (5.5) and (5.6), we obtain:

$$
\begin{aligned}
\left|J_{\varepsilon}^{X}\left[Q_{\varepsilon}\right]-\tilde{J}_{\varepsilon}^{X}\left[Q_{\varepsilon}\right]\right| \lesssim & \left(\left\|Q_{\varepsilon}\right\|_{L^{\infty}(\Omega)}^{3}+1\right) \cdot\left\|\nabla Q_{\varepsilon}\right\|_{L^{\infty}(\Omega)} \cdot \frac{2(r+q)}{p q r} \cdot \frac{p \varepsilon-\varepsilon^{\alpha}}{\varepsilon-\varepsilon^{\alpha}} \\
& \cdot X_{\varepsilon} \cdot \varepsilon^{3} \cdot \sqrt{\left(\varepsilon-\frac{\varepsilon^{\alpha}}{p}\right)^{2}+\left(\frac{\varepsilon^{\alpha}}{q}\right)^{2}+\left(\frac{\varepsilon^{\alpha}}{r}\right)^{2}}
\end{aligned}
$$

Using (5.7) and (6.11), we can rewrite the last inequality as follows:

$$
\left|J_{\varepsilon}^{X}\left[Q_{\varepsilon}\right]-\tilde{J}_{\varepsilon}^{X}\left[Q_{\varepsilon}\right]\right| \lesssim\left(\left\|Q_{\varepsilon}\right\|_{L^{\infty}(\Omega)}^{3}+1\right) \cdot\left\|\nabla Q_{\varepsilon}\right\|_{L^{\infty}(\Omega)} \cdot \sqrt{\left(\varepsilon-\frac{\varepsilon^{\alpha}}{p}\right)^{2}+\left(\frac{\varepsilon^{\alpha}}{q}\right)^{2}+\left(\frac{\varepsilon^{\alpha}}{r}\right)^{2}},
$$

since the term $\frac{2(r+q)}{p q r}$ can be bounded with an $\varepsilon$-independent constant. Now, because $p, q, r \geq 1$, we have:

$$
\frac{\varepsilon^{2 \alpha}}{p^{2}}, \frac{\varepsilon^{2 \alpha}}{q^{2}}, \frac{\varepsilon^{2 \alpha}}{r^{2}} \leq \varepsilon^{2 \alpha}
$$

and, because $\varepsilon>0$ and $\alpha \in(1,2)$, we also have:

$$
0<\varepsilon-\varepsilon^{\alpha} \leq \varepsilon-\frac{\varepsilon^{\alpha}}{k} \leq \varepsilon, \text { for } k \in\{p, q, r\} .
$$

Therefore, (6.13) becomes:

$$
\left|J_{\varepsilon}^{X}\left[Q_{\varepsilon}\right]-\tilde{J}_{\varepsilon}^{X}\left[Q_{\varepsilon}\right]\right| \lesssim\left(\left\|Q_{\varepsilon}\right\|_{L^{\infty}(\Omega)}^{3}+1\right) \cdot\left\|\nabla Q_{\varepsilon}\right\|_{L^{\infty}(\Omega)} \cdot \sqrt{\varepsilon^{2}+2 \varepsilon^{2 \alpha}}
$$

and using the same arguments for $J_{\varepsilon}^{Y}\left[Q_{\varepsilon}\right]$ and $J_{\varepsilon}^{Z}\left[Q_{\varepsilon}\right]$, we obtain:

$$
\left|J_{\varepsilon}^{\mathcal{T}}\left[Q_{\varepsilon}\right]-\tilde{J}_{\varepsilon}\left[Q_{\varepsilon}\right]\right| \lesssim\left(\left\|Q_{\varepsilon}\right\|_{L^{\infty}(\Omega)}^{3}+1\right) \cdot\left\|\nabla Q_{\varepsilon}\right\|_{L^{\infty}(\Omega)} \cdot \sqrt{\varepsilon^{2}+2 \varepsilon^{2 \alpha}}
$$

Using Lemma 6.3, we have:

$$
\begin{aligned}
\left\|Q_{\varepsilon}\right\|_{L^{\infty}(\Omega)}^{3} & \lesssim \varepsilon^{-3 \beta / 2}\left(\|Q\|_{H^{1}(\Omega)}+\|g\|_{L^{\infty}(\Omega)}\right)^{3} \\
\left\|\nabla Q_{\varepsilon}\right\|_{L^{\infty}(\Omega)} & \lesssim \varepsilon^{-3 \beta / 2}\left(\|Q\|_{H^{1}(\Omega)}+\|g\|_{W^{1, \infty}(\Omega)}\right) .
\end{aligned}
$$


Now, the constant involved by using the sign " $\lesssim$ " is going to depend also on the $L^{\infty}$-norms of $g$ and $\nabla g$, hence, relation (6.14) becomes:

$$
\left|J_{\varepsilon}^{\mathcal{T}}\left[Q_{\varepsilon}\right]-\tilde{J}_{\varepsilon}\left[Q_{\varepsilon}\right]\right| \lesssim \frac{\sqrt{\varepsilon^{2}+\varepsilon^{2 \alpha}}}{\varepsilon^{3 \beta}}\left(\|Q\|_{H^{1}(\Omega)}^{4}+1\right) \lesssim \sqrt{\varepsilon^{2(1-3 \beta)}+\varepsilon^{2(\alpha-3 \beta)}}\left(\|Q\|_{H^{1}(\Omega)}^{4}+1\right) .
$$

Since $\alpha \in(1,2)$, we have $1-3 \beta<\alpha-3 \beta$. Therefore, we can write the last inequality as follows:

$$
\left|J_{\varepsilon}^{\mathcal{T}}\left[Q_{\varepsilon}\right]-\tilde{J}_{\varepsilon}\left[Q_{\varepsilon}\right]\right| \lesssim \varepsilon^{1-3 \beta}\left(\|Q\|_{H^{1}(\Omega)}^{4}+1\right),
$$

since $\varepsilon \in(0,1)$.

In order to analyse better the second term from (6.12), which contains $\tilde{J}_{\varepsilon}\left[Q_{\varepsilon}\right]$ and $J_{0}\left[Q_{\varepsilon}\right]$, we analyse the first terms from (6.1) and (6.2):

$$
\begin{aligned}
\left|\frac{p \varepsilon-\varepsilon^{\alpha}}{\operatorname{pr}\left(\varepsilon-\varepsilon^{\alpha}\right)} \int_{\Omega} \Psi^{Y}\left(Q_{\varepsilon}\right) \mathrm{d} \mu_{\varepsilon}^{X}-\frac{1}{r} \int_{\Omega} \Psi^{Y}\left(Q_{\varepsilon}\right) \mathrm{d} x\right| & \leq \frac{p \varepsilon-\varepsilon^{\alpha}}{p r\left(\varepsilon-\varepsilon^{\alpha}\right)}\left|\int_{\Omega} \Psi^{Y}\left(Q_{\varepsilon}\right) \mathrm{d} \mu_{\varepsilon}^{X}-\int_{\Omega} \Psi^{Y}\left(Q_{\varepsilon}\right) \mathrm{d} x\right| \\
& +\left|\frac{p \varepsilon-\varepsilon^{\alpha}}{p r\left(\varepsilon-\varepsilon^{\alpha}\right)}-\frac{1}{r}\right| \cdot\left|\int_{\Omega} \Psi^{Y}\left(Q_{\varepsilon}\right) \mathrm{d} x\right| .
\end{aligned}
$$

As we have seen before, we have $\frac{p \varepsilon-\varepsilon^{\alpha}}{\operatorname{pr}\left(\varepsilon-\varepsilon^{\alpha}\right)} \searrow \frac{1}{r}$ and we have chosen $\varepsilon>0$ such that $\frac{1}{r} \leq \frac{p \varepsilon-\varepsilon^{\alpha}}{p r\left(\varepsilon-\varepsilon^{\alpha}\right)}<\frac{2}{r}$. Moreover, we have $\left|\frac{p \varepsilon-\varepsilon^{\alpha}}{\operatorname{pr}\left(\varepsilon-\varepsilon^{\alpha}\right)}-\frac{1}{r}\right|=\frac{\varepsilon^{\alpha}(p-1)}{\operatorname{pr}\left(\varepsilon-\varepsilon^{\alpha}\right)}$ and we can impose further conditions regarding the choice of $\varepsilon$, such that $\frac{\varepsilon^{\alpha}(p-1)}{\operatorname{pr}\left(\varepsilon-\varepsilon^{\alpha}\right)}<\varepsilon^{\alpha-1}$, which is equivalent to choosing $\varepsilon$ such that $\varepsilon^{\alpha-1}<1-\frac{1}{r}+\frac{1}{p r}$. Hence, we have:

$$
\left|\frac{p \varepsilon-\varepsilon^{\alpha}}{\operatorname{pr}\left(\varepsilon-\varepsilon^{\alpha}\right)} \int_{\Omega} \Psi^{Y}\left(Q_{\varepsilon}\right) \mathrm{d} \mu_{\varepsilon}^{X}-\frac{1}{r} \int_{\Omega} \Psi^{Y}\left(Q_{\varepsilon}\right) \mathrm{d} x\right| \leq \frac{2}{r}\left|\int_{\Omega} \Psi^{Y}\left(Q_{\varepsilon}\right) \mathrm{d} \mu_{\varepsilon}^{X}-\int_{\Omega} \Psi^{Y}\left(Q_{\varepsilon}\right) \mathrm{d} x\right|+\varepsilon^{\alpha-1}\left|\int_{\Omega} \Psi^{Y}\left(Q_{\varepsilon}\right) \mathrm{d} x\right| .
$$

Using the definition of $\mathbb{F}_{\varepsilon}$, we have:

$$
\left|\frac{p \varepsilon-\varepsilon^{\alpha}}{\operatorname{pr}\left(\varepsilon-\varepsilon^{\alpha}\right)} \int_{\Omega} \Psi^{Y}\left(Q_{\varepsilon}\right) \mathrm{d} \mu_{\varepsilon}^{X}-\frac{1}{r} \int_{\Omega} \Psi^{Y}\left(Q_{\varepsilon}\right) \mathrm{d} x\right| \leq \frac{2}{r} \cdot \mathbb{F}_{\varepsilon} \cdot\left\|\Psi^{Y}\left(Q_{\varepsilon}\right)\right\|_{W^{1, \infty}(\Omega)}+\varepsilon^{\alpha-1}\left\|\Psi^{Y}\left(Q_{\varepsilon}\right)\right\|_{L^{\infty}(\Omega)} .
$$

Using now the fact that now $Q_{\varepsilon} \in H_{g}^{1}\left(\Omega, \mathcal{S}_{0}\right)$, Lemma 6.1 and Lemma 6.2 , we obtain (also by moving the constant $\frac{2}{r}$ under the “ $\lesssim$ " sign):

$$
\begin{aligned}
\left|\frac{p \varepsilon-\varepsilon^{\alpha}}{p r\left(\varepsilon-\varepsilon^{\alpha}\right)} \int_{\Omega} \Psi^{Y}\left(Q_{\varepsilon}\right) \mathrm{d} \mu_{\varepsilon}^{X}-\frac{1}{r} \int_{\Omega} \Psi^{Y}\left(Q_{\varepsilon}\right) \mathrm{d} x\right| \lesssim \varepsilon\left(\left\|Q_{\varepsilon}\right\|_{L^{\infty}(\Omega)}^{4}+1\right) \\
+\varepsilon\left(\left\|Q_{\varepsilon}\right\|_{L^{\infty}(\Omega)}^{3}+1\right) \cdot\left\|\nabla Q_{\varepsilon}\right\|_{L^{\infty}(\Omega)}+\varepsilon^{\alpha-1}\left(\left\|Q_{\varepsilon}\right\|_{L^{\infty}(\Omega)}^{4}+1\right),
\end{aligned}
$$

Applying Lemma 6.3, we get:

$$
\begin{gathered}
\left|\frac{p \varepsilon-\varepsilon^{\alpha}}{\operatorname{pr}\left(\varepsilon-\varepsilon^{\alpha}\right)} \int_{\Omega} \Psi^{Y}\left(Q_{\varepsilon}\right) \mathrm{d} \mu_{\varepsilon}^{X}-\frac{1}{r} \int_{\Omega} \Psi^{Y}\left(Q_{\varepsilon}\right) \mathrm{d} x\right| \lesssim \varepsilon\left(\varepsilon^{-2 \beta}\left(\|Q\|_{H^{1}(\Omega)}+\|g\|_{L^{\infty}(\Omega)}\right)^{4}+1\right) \\
+\varepsilon\left(\varepsilon^{-3 \beta / 2}\left(\|Q\|_{H^{1}(\Omega)}+\|g\|_{L^{\infty}(\Omega)}\right)^{3}+1\right) \cdot \varepsilon^{-3 \beta / 2}\left(\|Q\|_{H^{1}(\Omega)}+\|g\|_{W^{1, \infty}(\Omega)}\right)
\end{gathered}
$$




$$
+\varepsilon^{\alpha-1}\left(\varepsilon^{-2 \beta}\left(\|Q\|_{H^{1}(\Omega)}+\|g\|_{L^{\infty}(\Omega)}\right)^{4}+1\right)
$$

Moving the terms $\|g\|_{L^{\infty}(\Omega)}$ and $\|g\|_{W^{1, \infty}(\Omega)}$ under the "¿" sign and using the fact that $\beta>0$ and $\varepsilon \in(0,1)$, we have:

$$
\left|\frac{p \varepsilon-\varepsilon^{\alpha}}{\operatorname{pr}\left(\varepsilon-\varepsilon^{\alpha}\right)} \int_{\Omega} \Psi^{Y}\left(Q_{\varepsilon}\right) \mathrm{d} \mu_{\varepsilon}^{X}-\frac{1}{r} \int_{\Omega} \Psi^{Y}\left(Q_{\varepsilon}\right) \mathrm{d} x\right| \lesssim\left(\varepsilon^{1-2 \beta}+\varepsilon^{1-3 \beta}+\varepsilon^{\alpha-2 \beta-1}\right)\left(\|Q\|_{H^{1}(\Omega)}^{4}+1\right) .
$$

Applying the same technique for the other five terms from $J_{0}$ and $\tilde{J}_{\varepsilon}$, which are in (6.1) and (6.2), we obtain:

$$
\left|\tilde{J}_{\varepsilon}\left[Q_{\varepsilon}\right]-J_{0}\left[Q_{\varepsilon}\right]\right| \lesssim\left(\varepsilon^{1-2 \beta}+\varepsilon^{1-3 \beta}+\varepsilon^{\alpha-2 \beta-1}\right)\left(\|Q\|_{H^{1}(\Omega)}^{4}+1\right)
$$

and using once again that $\beta>0$ and $\varepsilon \in(0,1)$, we can write:

$$
\left|\tilde{J}_{\varepsilon}\left[Q_{\varepsilon}\right]-J_{0}\left[Q_{\varepsilon}\right]\right| \lesssim\left(\varepsilon^{1-3 \beta}+\varepsilon^{\alpha-2 \beta-1}\right)\left(\|Q\|_{H^{1}(\Omega)}^{4}+1\right) .
$$

Moving now to the last term from (6.12), which is $\left|J_{0}\left[Q_{\varepsilon}\right]-J_{0}[Q]\right|$, we once again analyse every difference that can be formed with the six terms from the definition of (6.1). Hence:

$$
\left|\int_{\Omega} \frac{1}{r} \Psi^{Y}\left(Q_{\varepsilon}\right) \mathrm{d} x-\int_{\Omega} \frac{1}{r} \Psi^{Y}(Q) \mathrm{d} x\right| \leq \frac{1}{r} \int_{\Omega}\left|\Psi^{Y}\left(Q_{\varepsilon}\right)-\Psi^{Y}(Q)\right| \mathrm{d} x
$$

Using Lemma 6.1 and moving the constant $\frac{1}{r}$ under the “ $\lesssim$ "sign, we have:

$$
\begin{aligned}
\left|\int_{\Omega} \frac{1}{r} \Psi^{Y}\left(Q_{\varepsilon}\right) \mathrm{d} x-\int_{\Omega} \frac{1}{r} \Psi^{Y}(Q) \mathrm{d} x\right| & \lesssim \int_{\Omega}\left(|Q|^{3}+\left|Q_{\varepsilon}\right|^{3}+1\right)\left|Q-Q_{\varepsilon}\right| \mathrm{d} x \\
& \lesssim\left(\int_{\Omega}\left(|Q|^{3}+\left|Q_{\varepsilon}\right|^{3}+1\right)^{2} \mathrm{~d} x\right)^{1 / 2} \cdot\left(\int_{\Omega}\left|Q-Q_{\varepsilon}\right|^{2} \mathrm{~d} x\right)^{1 / 2} \\
& \lesssim\left(\int_{\Omega}\left(|Q|^{6}+\left|Q_{\varepsilon}\right|^{6}+1\right) \mathrm{d} x\right)^{1 / 2} \cdot\left\|Q-Q_{\varepsilon}\right\|_{L^{2}(\Omega)} \\
& \lesssim\left(\|Q\|_{L^{6}(\Omega)}^{3}+\left\|Q_{\varepsilon}\right\|_{L^{6}(\Omega)}^{3}+1\right) \cdot\left\|Q-Q_{\varepsilon}\right\|_{L^{2}(\Omega)} .
\end{aligned}
$$

The sequence $\left(Q_{\varepsilon}\right)_{\varepsilon>0}$ is bounded in $L^{6}(\Omega)$, due to the continuous Sobolev embedding $H^{1}(\Omega) \hookrightarrow L^{6}(\Omega)$ and to Lemma 6.3. Using once again Lemma 6.3 to control $\left\|Q-Q_{\varepsilon}\right\|_{L^{2}(\Omega)}$, we obtain:

$$
\left|\int_{\Omega} \frac{1}{r} \Psi^{Y}\left(Q_{\varepsilon}\right) \mathrm{d} x-\int_{\Omega} \frac{1}{r} \Psi^{Y}(Q) \mathrm{d} x\right| \lesssim \varepsilon^{\beta}\left(\|Q\|_{H^{1}(\Omega)}^{4}+1\right),
$$

hence

$$
\mid J_{0}\left[Q_{\varepsilon}-J_{0}[Q] \mid \lesssim \varepsilon^{\beta}\left(\|Q\|_{H^{1}(\Omega)}^{4}+1\right) .\right.
$$

Combining now relations (6.15), (6.16) and (6.17), we obtain:

$$
\left|J_{\varepsilon}^{\mathcal{T}}\left[Q_{\varepsilon}\right]-J_{0}[Q]\right| \lesssim\left(\varepsilon^{1-3 \beta}+\varepsilon^{\alpha-2 \beta-1}+\varepsilon^{\beta}\right)\left(\|Q\|_{H^{1}(\Omega)}^{4}+1\right) .
$$


Now we need to find a suitable value for $\beta>0$ such that we can put the minimum positive value between the exponents $1-3 \beta, \alpha-2 \beta-1$ and $\beta$, in order to obtain the best rate of convergence. This is equivalent to choosing $\beta=\min \left\{\frac{1}{4}, \frac{\alpha-1}{3}, 2-\alpha\right\}$. If $\alpha \in\left(1, \frac{7}{4}\right]$, then $\beta=\frac{\alpha-1}{3}$. If $\alpha \in\left(\frac{7}{4}, 2\right)$, then $\beta=2-\alpha$. This implies that:

$$
\left|J_{\varepsilon}^{\mathcal{T}}\left[Q_{\varepsilon}\right]-J_{0}[Q]\right| \lesssim \varepsilon^{m_{0}}\left(\|Q\|_{H^{1}(\Omega)}^{4}+1\right)
$$

where $m_{0}=\min \left\{\frac{\alpha-1}{3}, 2-\alpha\right\}$ for $\alpha \in(1,2)$.

\section{Appendix A.}

\section{A.1 Constructing the cubic microlattice}

In this subsection, we provide more details regarding the construction of the "connecting parallelepipeds", which are the grey parallelepipeds from Figure 1.

In each of the points from $\mathcal{Y}_{\varepsilon}$ we construct a parallelepiped that connects the parallelepipeds $\mathcal{C}_{\varepsilon}^{i}$ and $\mathcal{C}_{\varepsilon}^{j}$, where $i, j \in \overline{1, N_{\varepsilon}}$ such that $\left|x_{\varepsilon}^{i}-x_{\varepsilon}^{j}\right|=\varepsilon$.

If $x_{\varepsilon}^{i}-x_{\varepsilon}^{j}= \pm(\varepsilon, 0,0)^{T}$, then let:

$-X_{\varepsilon}=\operatorname{card}\left(\left\{(i, j) \in{\overline{1, N_{\varepsilon}}}^{2} \mid x_{\varepsilon}^{i}-x_{\varepsilon}^{j}=(\varepsilon, 0,0)^{T}, i<j\right\}\right)$

$-\Upsilon_{\varepsilon}^{x}:\left\{(i, j) \in{\overline{1, N_{\varepsilon}}}^{2} \mid x_{\varepsilon}^{i}-x_{\varepsilon}^{j}=(\varepsilon, 0,0)^{T}, i<j\right\} \rightarrow \overline{1, X_{\varepsilon}}$ a bijection;

$-y_{\varepsilon}^{x, k}=\frac{1}{2}\left(x_{\varepsilon}^{i}+x_{\varepsilon}^{j}\right)$, where $k=\Upsilon_{\varepsilon}^{x}(i, j)$;

$-\mathcal{Y}_{\varepsilon}^{x}=\left\{y_{\varepsilon}^{x, k} \in \mathcal{Y}_{\varepsilon} \mid y_{\varepsilon}^{x, k}=\frac{1}{2}\left(x_{\varepsilon}^{i}+x_{\varepsilon}^{j}\right), k=\Upsilon_{\varepsilon}^{x}(i, j)\right\} ;$

$-\mathcal{P}_{\varepsilon}^{x, k}$ the "connecting parallelepiped" centered in $y_{\varepsilon}^{x, k}$, defined by $\mathcal{P}_{\varepsilon}^{x, k}=y_{\varepsilon}^{x, k}+T_{x} \mathcal{C}^{\alpha}$, where

$$
T_{x} \mathcal{C}^{\alpha}=\left[-\frac{p \varepsilon-\varepsilon^{\alpha}}{2 p}, \frac{p \varepsilon-\varepsilon^{\alpha}}{2 p}\right] \times\left[-\frac{\varepsilon^{\alpha}}{2 q}, \frac{\varepsilon^{\alpha}}{2 q}\right] \times\left[-\frac{\varepsilon^{\alpha}}{2 r}, \frac{\varepsilon^{\alpha}}{2 r}\right] ;
$$

$-\mathcal{T}_{x}^{k}$ be the union of the four transparent faces of $\mathcal{P}_{\varepsilon}^{x, k}$ that have the length equal to $\frac{p \varepsilon-\varepsilon^{\alpha}}{p}$, which are represented in Figure A.1a.

If $x_{\varepsilon}^{i}-x_{\varepsilon}^{j}= \pm(0, \varepsilon, 0)^{T}$, then let:

$$
\begin{aligned}
& -Y_{\varepsilon}=\operatorname{card}\left(\left\{(i, j) \in{\overline{1, N_{\varepsilon}}}^{2} \mid x_{\varepsilon}^{i}-x_{\varepsilon}^{j}=(0, \varepsilon, 0)^{T}, i<j\right\}\right) \\
& -\Upsilon_{\varepsilon}^{y}:\left\{(i, j) \in{\overline{1, N_{\varepsilon}}}^{2} \mid x_{\varepsilon}^{i}-x_{\varepsilon}^{j}=(0, \varepsilon, 0)^{T}, i<j\right\} \rightarrow \overline{1, Y_{\varepsilon}} \text { a bijection; } \\
& -y_{\varepsilon}^{y, l}=\frac{1}{2}\left(x_{\varepsilon}^{i}+x_{\varepsilon}^{j}\right), \text { where } l=\Upsilon_{\varepsilon}^{y}(i, j) ; \\
& -\mathcal{Y}_{\varepsilon}^{y}=\left\{y_{\varepsilon}^{y, l} \in \mathcal{Y}_{\varepsilon} \mid y_{\varepsilon}^{y, l}=\frac{1}{2}\left(x_{\varepsilon}^{i}+x_{\varepsilon}^{j}\right), l=\Upsilon_{\varepsilon}^{y}(i, j)\right\} ;
\end{aligned}
$$

$-\mathcal{P}_{\varepsilon}^{y, l}$ the "connecting parallelepiped" centered in $y_{\varepsilon}^{y, l}$, defined by $\mathcal{P}_{\varepsilon}^{y, l}=y_{\varepsilon}^{y, l}+T_{y} \mathcal{C}^{\alpha}$, where

$$
T_{y} \mathcal{C}^{\alpha}=\left[-\frac{\varepsilon^{\alpha}}{2 p}, \frac{\varepsilon^{\alpha}}{2 p}\right] \times\left[-\frac{q \varepsilon-\varepsilon^{\alpha}}{2 q}, \frac{q \varepsilon-\varepsilon^{\alpha}}{2 q}\right] \times\left[-\frac{\varepsilon^{\alpha}}{2 r}, \frac{\varepsilon^{\alpha}}{2 r}\right] ;
$$




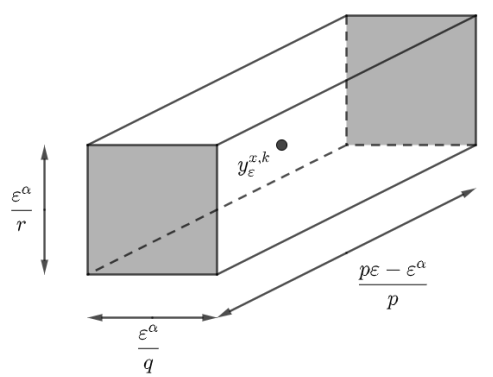

(a) The "connecting parallelepiped" $\mathcal{P}_{\varepsilon}^{x, k}$, with the center in $y_{\varepsilon}^{x, k}$, with lateral transparent faces $\mathcal{T}_{x}^{k}$.

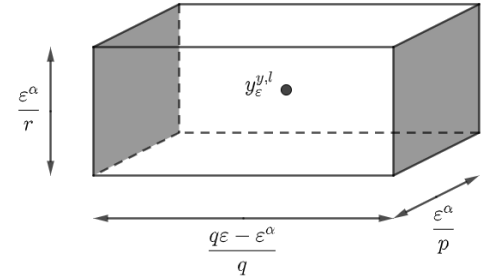

(b) The "connecting parallelepiped" $\mathcal{P}_{\varepsilon}^{y, l}$, with the center in $y_{\varepsilon}^{y, l}$, with lateral transparent faces $\mathcal{T}_{y}^{l}$.

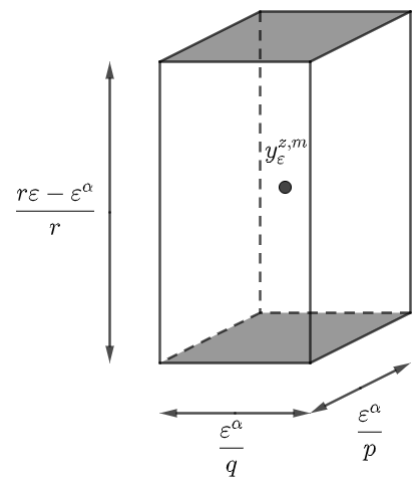

(c) The "connecting parallelepiped" $\mathcal{P}_{\varepsilon}^{z, m}$, with the center in $y_{\varepsilon}^{z, m}$, with lateral transparent faces $\mathcal{T}_{z}^{m}$.

Figure A.1. The three types of "connecting parallelepipeds" with centers from $\mathcal{Y}_{\varepsilon}$.

$-\mathcal{T}_{y}^{l}$ be the union of the four transparent faces of $\mathcal{P}_{\varepsilon}^{y, l}$ that have the length equal to $\frac{q \varepsilon-\varepsilon^{\alpha}}{q}$, which are represented in Figure A.1b.

If $x_{\varepsilon}^{i}-x_{\varepsilon}^{j}= \pm(0,0, \varepsilon)^{T}$, then let:

$-Z_{\varepsilon}=\operatorname{card}\left(\left\{(i, j) \in{\overline{1, N_{\varepsilon}}}^{2} \mid x_{\varepsilon}^{i}-x_{\varepsilon}^{j}=(0,0, \varepsilon)^{T}, i<j\right\}\right)$

$-\Upsilon_{\varepsilon}^{y}:\left\{(i, j) \in \overline{1, N_{\varepsilon}^{2}} \mid x_{\varepsilon}^{i}-x_{\varepsilon}^{j}=(0,0, \varepsilon)^{T}, i<j\right\} \rightarrow \overline{1, Z_{\varepsilon}}$ a bijection;

$-y_{\varepsilon}^{z, m}=\frac{1}{2}\left(x_{\varepsilon}^{i}+x_{\varepsilon}^{j}\right)$, where $m=\Upsilon_{\varepsilon}^{z}(i, j)$;

$-\mathcal{Y}_{\varepsilon}^{z}=\left\{y_{\varepsilon}^{z, m} \in \mathcal{Y}_{\varepsilon} \mid y_{\varepsilon}^{z, m}=\frac{1}{2}\left(x_{\varepsilon}^{i}+x_{\varepsilon}^{j}\right), m=\Upsilon_{\varepsilon}^{z}(i, j)\right\} ;$

$-\mathcal{P}_{\varepsilon}^{z, m}$ the "connecting parallelepiped" centered in $y_{\varepsilon}^{z, m}$, defined by $\mathcal{P}_{\varepsilon}^{z, m}=y_{\varepsilon}^{z, m}+T_{z} \mathcal{C}^{\alpha}$, where

$$
T_{z} \mathcal{C}^{\alpha}=\left[-\frac{\varepsilon^{\alpha}}{2 p}, \frac{\varepsilon^{\alpha}}{2 p}\right] \times\left[-\frac{\varepsilon^{\alpha}}{2 q}, \frac{\varepsilon^{\alpha}}{2 q}\right] \times\left[-\frac{r \varepsilon-\varepsilon^{\alpha}}{2 r}, \frac{r \varepsilon-\varepsilon^{\alpha}}{2 r}\right]
$$

$-\mathcal{T}_{z}^{m}$ be the union of the four transparent faces of $\mathcal{P}_{\varepsilon}^{z, m}$ that have the length equal to $\frac{r \varepsilon-\varepsilon^{\alpha}}{r}$, which are represented in Figure A.1c.

Remark A.1. We can see that we need to set $\varepsilon^{\alpha-1}<\min \{p, q, r\}$, otherwise we have $\frac{\varepsilon^{\alpha-1}}{p} \geq 1 \Rightarrow \frac{\varepsilon^{\alpha}}{2 p} \geq \frac{\varepsilon}{2}$ and, in the same way, $\frac{\varepsilon^{\alpha}}{2 q} \geq \frac{\varepsilon}{2}$ and $\frac{\varepsilon^{\alpha}}{2 r} \geq \frac{\varepsilon}{2}$, hence the inclusions from the family $\mathcal{C}_{\varepsilon}$, which represents the set of all "inner parallelepipeds", are not disjoint anymore and they overlap. More specifically, the "connecting parallelepipeds" cannot be constructed anymore. Since the parameters $p, q$ and $r$ are fixed and we are interested in what happens when $\varepsilon \rightarrow 0$, then the condition $\varepsilon^{\alpha-1}<\min \{p, q, r\}$ implies that $\alpha \geq 1$. If $\alpha=1$, then it is easy to see that the volume of the scaffold does not tend to zero as $\varepsilon \rightarrow 0$, so we are not in the dilute regime anymore. 


\section{A.2 Volume and surface area of the scaffold}

Proposition A.2. The volume of the scaffold $\mathcal{N}_{\varepsilon}$ tends to 0 as $\varepsilon \rightarrow 0$.

Proof. According to (2.1), (2.5), (2.6), (2.8), (A.1), (A.7) and (A.12), we have:

$$
-N_{\varepsilon}<\frac{L_{0} l_{0} h_{0}}{\varepsilon^{3}} ; \quad-X_{\varepsilon}<\left(\frac{L_{0}}{\varepsilon}-1\right) \cdot \frac{l_{0} h_{0}}{\varepsilon^{2}} ; \quad-Y_{\varepsilon}<\left(\frac{l_{0}}{\varepsilon}-1\right) \frac{L_{0} h_{0}}{\varepsilon^{2}} ; \quad-Z_{\varepsilon}<\left(\frac{h_{0}}{\varepsilon}-1\right) \frac{L_{0} l_{0}}{\varepsilon^{2}} .
$$

Furthermore, we have:

$$
\left|\mathcal{N}_{\varepsilon}\right|=N_{\varepsilon} \cdot \frac{\varepsilon^{3 \alpha}}{p q r}+X_{\varepsilon} \cdot \frac{\varepsilon^{2 \alpha}}{p q r}\left(p \varepsilon-\varepsilon^{\alpha}\right)+Y_{\varepsilon} \cdot \frac{\varepsilon^{2 \alpha}}{p q r}\left(q \varepsilon-\varepsilon^{\alpha}\right)+Z_{\varepsilon} \cdot \frac{\varepsilon^{2 \alpha}}{p q r}\left(r \varepsilon-\varepsilon^{\alpha}\right),
$$

where $\frac{\varepsilon^{3 \alpha}}{p q r}$ represents the volume of an "inner parallelepiped" defined in (2.7) and $\frac{\varepsilon^{2 \alpha}}{p q r}\left(p \varepsilon-\varepsilon^{\alpha}\right), \frac{\varepsilon^{2 \alpha}}{p q r}\left(q \varepsilon-\varepsilon^{\alpha}\right)$ and

$\frac{\varepsilon^{2 \alpha}}{p q r}\left(r \varepsilon-\varepsilon^{\alpha}\right)$ represent the volume of a "connecting parallelepiped" $\mathcal{P}_{\varepsilon}^{x, k}, \mathcal{P}_{\varepsilon}^{y, l}$ and, respectively, $\mathcal{P}_{\varepsilon}^{z, m}$, which are defined in (A.4), (A.9) and (A.14). Hence:

$$
\begin{aligned}
\left|\mathcal{N}_{\varepsilon}\right|< & \frac{L_{0} l_{0} h_{0}(p+q+r)}{p q r} \varepsilon^{2(\alpha-1)}-2 \frac{L_{0} l_{0} h_{0}}{p q r} \varepsilon^{3(\alpha-1)}-\left(\frac{L_{0} l_{0}}{p q}+\frac{L_{0} h_{0}}{p r}+\frac{l_{0} h_{0}}{q r}\right) \varepsilon^{2 \alpha-1} \\
& +\frac{L_{0} l_{0}+L_{0} h_{0}+l_{0} h_{0}}{p q r} \varepsilon^{3 \alpha-2} .
\end{aligned}
$$

Because $\alpha>1$, according to $\left(A_{2}\right)$, then $2(\alpha-1)>0,3(\alpha-1)>0,2 \alpha-1>0$ and $3 \alpha-2>0$, therefore $\left|\mathcal{N}_{\varepsilon}\right| \rightarrow 0$ as $\varepsilon \rightarrow 0$.

Proposition A.3. There exists an $\varepsilon$-independent constant $C_{s}=C_{s}(p, q, r, \Omega)>0$ such that:

$$
\lim _{\varepsilon \rightarrow 0} \frac{\varepsilon^{3}}{\varepsilon^{\alpha}\left(\varepsilon-\varepsilon^{\alpha}\right)}\left|\partial \mathcal{N}_{\varepsilon}\right|<C_{s}
$$

Proof. Using the same tehnique as in Proposition A.2, we have, considering relations (A.6), (A.11), (A.16) and (4.6):

$$
\begin{aligned}
\left|\partial \mathcal{N}_{\varepsilon}\right|< & N_{\varepsilon} \cdot \varepsilon^{2 \alpha} \cdot \frac{2(p+q+r)}{p q r}+X_{\varepsilon} \cdot \varepsilon^{\alpha}\left(p \varepsilon-\varepsilon^{\alpha}\right) \cdot \frac{2(q+r)}{p q r} \\
& +Y_{\varepsilon} \cdot \varepsilon^{\alpha}\left(q \varepsilon-\varepsilon^{\alpha}\right) \cdot \frac{2(p+r)}{p q r}+Z_{\varepsilon} \cdot \varepsilon^{\alpha}\left(r \varepsilon-\varepsilon^{\alpha}\right) \cdot \frac{2(p+q)}{p q r} \\
< & C\left(p, q, r, L_{0}, l_{0}, h_{0}\right) \cdot \varepsilon^{\alpha-3} \cdot\left((p+q+r) \varepsilon-2 \varepsilon^{\alpha}\right) .
\end{aligned}
$$

Hence

$$
\lim _{\varepsilon \rightarrow 0} \frac{\varepsilon^{3}}{\varepsilon^{\alpha}\left(\varepsilon-\varepsilon^{\alpha}\right)}\left|\partial \mathcal{N}_{\varepsilon}\right|<C\left(p, q, r, L_{0}, l_{0}, h_{0}\right) \cdot \lim _{\varepsilon \rightarrow 0} \frac{(p+q+r) \varepsilon-2 \varepsilon^{\alpha}}{\varepsilon-\varepsilon^{\alpha}}<+\infty
$$

We denote $C_{s}$ the constant obtained in the last inequality.

Proposition A.4. Let $\partial \mathcal{N}_{\varepsilon}^{\mathcal{S}}$ be the set defined in (4.6). Then, for $\varepsilon \rightarrow 0$, we have $\left|\partial \mathcal{N}_{\varepsilon}^{\mathcal{S}}\right| \rightarrow 0$. 
Proof. According to (4.6), we have:

$$
\partial \mathcal{N}_{\varepsilon}^{\mathcal{S}}=\left(\bigcup_{i=1}^{N_{\varepsilon, 2}} \mathcal{S}^{i}\right)
$$

therefore, we can write:

$$
\left|\partial \mathcal{N}_{\varepsilon}^{\mathcal{S}}\right| \leq \sum_{i=1}^{N_{\varepsilon, 2}}\left|\mathcal{S}^{i}\right| \leq \sum_{i=1}^{N_{\varepsilon, 2}}\left|\mathcal{C}_{\varepsilon}^{i}\right| \leq \sum_{i=1}^{N_{\varepsilon, 2}} \frac{\varepsilon^{2 \alpha}(p+q+r)}{p q r} \leq \frac{\varepsilon^{2 \alpha}(p+q+r)}{p q r} \cdot N_{\varepsilon, 2}
$$

where we have used (4.5) and (2.7). Since $N_{\varepsilon, 2}$ counts only the "inner parallelepipeds" that are close to the boundary of $\Omega$ (meaning that these objects have less than 6 adjacent "connecting parallelepipeds; also, see (4.4)), then we can write:

$$
N_{\varepsilon, 2}<\frac{L_{0} \cdot l_{0}}{\varepsilon^{2}}+\frac{L_{0} \cdot h_{0}}{\varepsilon^{2}}+\frac{l_{0} \cdot h_{0}}{\varepsilon^{2}},
$$

where $L_{0}, l_{0}$ and $h_{0}$ are defined in (2.1) and they describe the parallelepiped that contains the entire domain $\Omega$. From here, we obtain:

$$
\left|\partial \mathcal{N}_{\varepsilon}^{\mathcal{S}}\right| \leq \frac{\varepsilon^{2 \alpha}(p+q+r)}{p q r} \cdot \frac{L_{0} l_{0}+l_{0} h_{0}+h_{0} L_{0}}{\varepsilon^{2}} \lesssim \varepsilon^{2(\alpha-1)} \rightarrow 0, \text { as } \varepsilon \rightarrow 0
$$

since $\alpha>1$.

\section{A.3 Constructing an explicit extension of $Q$ inside the scaffold}

The aim of this subsection is to prove that there exists a function $v \in H^{1}(\Omega)$ such that $v=Q$ on $\partial \mathcal{N}_{\varepsilon}, v=Q$ in $\Omega_{\varepsilon}$ and $\|\nabla v\|_{L^{2}(\Omega)} \lesssim\|\nabla Q\|_{L^{2}\left(\Omega_{\varepsilon}\right)}$.

In order to prove it, we first construct an explicit extension $u: \Omega_{\varepsilon} \cup \mathcal{N}_{\varepsilon}^{\mathcal{T}} \rightarrow \mathcal{S}_{0}$ such that $u \in H^{1}\left(\Omega_{\varepsilon} \cup \mathcal{N}_{\varepsilon}^{\mathcal{T}}, \mathcal{S}_{0}\right)$ and there exists a constant $C$, independent of $\varepsilon$, for which we have

$$
\|\nabla u\|_{L^{2}\left(\mathcal{N}_{\varepsilon}^{\mathcal{T}}\right)} \leq C\|\nabla Q\|_{L^{2}\left(\Omega_{\varepsilon}\right)},
$$

which implies $\|\nabla u\|_{L^{2}\left(\Omega_{\varepsilon} \cup \mathcal{N}_{\varepsilon}^{\mathcal{T}}\right)} \leq C\|\nabla Q\|_{L^{2}\left(\Omega_{\varepsilon}\right)}$.

Then we construct $v: \Omega \rightarrow \mathcal{S}_{0}$ such that $v \in H^{1}(\Omega), v \equiv u$ on $\Omega_{\varepsilon} \cup \mathcal{N}_{\varepsilon}^{\mathcal{T}}, v=u$ on $\partial \mathcal{N}_{\varepsilon}^{\mathcal{T}}$ and there exists a constant $c$ such that:

$$
\|\nabla v\|_{L^{2}(\Omega)} \leq c\|\nabla u\|_{L^{2}\left(\Omega_{\varepsilon} \cup \mathcal{N}_{\varepsilon}^{\mathcal{T}}\right)},
$$

which implies that $\|\nabla v\|_{L^{2}(\Omega)} \lesssim\|\nabla Q\|_{L^{2}\left(\Omega_{\varepsilon}\right)}$, using the properties mentioned for $u$.

We prove first the following result.

Lemma A.5. Let $z_{0}, a, b \in \mathbb{R}, a, b, z_{0}>0$ and let $A_{a, b}=\{(\rho \cos \theta, \rho \sin \theta): 0 \leq \theta<2 \pi, a<\rho<b\}$ be a two dimensional annulus, $B_{a}=\{(\rho \cos \theta, \rho \sin \theta): 0 \leq \theta<2 \pi, 0 \leq \rho<a\}$ be a two dimensional ball with radius a, $\mathcal{A}_{a, b}^{z_{0}}=A_{a, b} \times\left(-z_{0}, z_{0}\right) \subset \mathbb{R}^{3}$ and $\mathcal{B}_{a}^{z_{0}}=B_{a} \times\left(-z_{0}, z_{0}\right) \subset \mathbb{R}^{3}$ be a three dimensional cylinder. 
Let $Q \in H^{1}\left(\mathcal{A}_{1,2}^{z_{0}}, \mathcal{S}_{0}\right)$. Then the function $u: \mathcal{B}_{1}^{z_{0}} \rightarrow \mathcal{S}_{0}$ defined for any $z \in\left(-z_{0}, z_{0}\right)$ as

$$
u(x, y, z)=\left\{\begin{array}{l}
\varphi\left(\sqrt{x^{2}+y^{2}}\right) Q\left(\left(\frac{2}{\sqrt{x^{2}+y^{2}}}-1\right) x,\left(\frac{2}{\sqrt{x^{2}+y^{2}}}-1\right) y, z\right) \\
\quad+\left(1-\varphi\left(\sqrt{x^{2}+y^{2}}\right)\right) f_{A_{1,3 / 2}} Q(s, t, z) \mathrm{d}(s, t), \text { for } \frac{1}{2} \leq \sqrt{x^{2}+y^{2}}<1 \\
f_{A_{1,3 / 2}} Q(s, t, z) \mathrm{d}(s, t), \text { for } 0 \leq \sqrt{x^{2}+y^{2}} \leq \frac{1}{2}
\end{array}\right.
$$

is from $H^{1}\left(\mathcal{B}_{1}^{z_{0}}, \mathcal{S}_{0}\right)$, where $\varphi \in C_{c}^{\infty}\left(\left(\frac{1}{2}, \frac{3}{2}\right)\right)$ is the following bump function defined as

$$
\varphi(\rho)=\left\{\begin{array}{l}
\exp \left\{4-\frac{4}{(2 \rho-1)(3-2 \rho)}\right\}, \forall \rho \in\left(\frac{1}{2}, \frac{3}{2}\right), \\
0, \forall \rho \in \mathbb{R} \backslash\left(\frac{1}{2}, \frac{3}{2}\right)
\end{array},\right.
$$

the product $\varphi(\rho) Q$ represents product between a scalar and a Q-tensor and $f$ represents the average integral sign. Moreover, there exists a constant $c>0$, independent of $z_{0}$, such that: $\left\|u_{t}\right\|_{L^{2}\left(\mathcal{B}_{1}^{z_{0}}\right)} \leq c\left\|Q_{t}\right\|_{L^{2}\left(\mathcal{A}_{1,3 / 2}^{z_{0}}\right)}$ for any $t \in\{x, y, z\}$, where $u_{t}$ represents the partial derivative of $u$ with respect to $t$, and:

$$
\|\nabla u\|_{L^{2}\left(\mathcal{B}_{1}^{z_{0}}\right)} \leq c\|\nabla Q\|_{L^{2}\left(\mathcal{A}_{1,3 / 2}^{z_{0}}\right)}
$$

Proof. First of all, we can assume without loss of generality that $Q$ and $u$ are scalar functions, instead of $Q$-tensors. Hence, we prove this lemma for each of the component of $Q$ and $u$.

Let $T: A_{1 / 2,1} \rightarrow A_{1,3 / 2}$ be the reflection defined as:

$$
T(x, y)=\left(\left(\frac{2}{\sqrt{x^{2}+y^{2}}}-1\right) x,\left(\frac{2}{\sqrt{x^{2}+y^{2}}}-1\right) y\right):=\left(x^{\prime}, y^{\prime}\right), \forall(x, y) \in A_{1 / 2,1}
$$

Then $T$ is invertible and also bi-Lipschitz.

Let $Q \in H^{1}\left(\mathcal{A}_{1,3 / 2}^{z_{0}}\right)$ and $u$ defined by (A.17). By Theorem 3.17 from [1], we can approximate the function $Q \in H^{1}\left(\mathcal{A}_{1,3 / 2}^{z_{0}}\right)$ with smooth functions from $C^{\infty}\left(\overline{\mathcal{A}_{1,3 / 2}^{z_{0}}}\right)$.

Let $\left(Q_{k}\right)_{k \geq 1} \subset C^{\infty}\left(\overline{\mathcal{A}_{1,3 / 2}^{z_{0}}}\right)$ such that $Q_{k} \rightarrow Q$ strongly in $H^{1}\left(\mathcal{A}_{1,3 / 2}^{z_{0}}\right)$ and, for any $k \geq 1$, let $u_{k}: \overline{\mathcal{B}_{1}^{z_{0}}} \rightarrow \mathbb{R}$ defined for all $z \in\left(-z_{0}, z_{0}\right)$ as:

$$
u_{k}(x, y, z)=\left\{\begin{array}{l}
\varphi\left(\sqrt{x^{2}+y^{2}}\right) Q_{k}(T(x, y), z) \\
\quad+\left(1-\varphi\left(\sqrt{x^{2}+y^{2}}\right)\right) f_{A_{1,3 / 2}} Q_{k}(s, t, z) \mathrm{d}(s, t), \text { for } \frac{1}{2} \leq \sqrt{x^{2}+y^{2}} \leq 1 \\
f_{A_{1,3 / 2}} Q_{k}(s, t, z) d(s, t), \text { for } 0 \leq \sqrt{x^{2}+y^{2}} \leq \frac{1}{2}
\end{array}\right.
$$

By the above definition, we have that $u_{k} \in C^{0}\left(\overline{\mathcal{B}_{1}^{z_{0}}}\right)$ and that: 


$$
\frac{\partial u_{k}}{\partial x}(x, y, z)=\left\{\begin{array}{l}
\frac{x}{\sqrt{x^{2}+y^{2}}} \cdot \varphi^{\prime}\left(\sqrt{x^{2}+y^{2}}\right) \cdot\left(Q_{k}\left(x^{\prime}, y^{\prime}, z\right)-f_{A_{1,3 / 2}} Q_{k}(s, t, z) \mathrm{d}(s, t)\right) \\
\quad+\left(\frac{2 y^{2}}{\left(\sqrt{\left.x^{2}+y^{2}\right)}\right)^{3}}-1\right) \cdot \varphi\left(\sqrt{x^{2}+y^{2}}\right) \cdot\left(\frac{\partial Q_{k}}{\partial x^{\prime}}\left(x^{\prime}, y^{\prime}, z\right)\right) \\
-\frac{2 x y}{\left(\sqrt{x^{2}+y^{2}}\right)^{3}} \cdot \varphi\left(\sqrt{x^{2}+y^{2}}\right) \cdot\left(\frac{\partial Q_{k}}{\partial y^{\prime}}\left(x^{\prime}, y^{\prime}, z\right)\right), \text { for } \frac{1}{2} \leq \sqrt{x^{2}+y^{2}}<1 \\
0, \text { for } 0 \leq \sqrt{x^{2}+y^{2}} \leq \frac{1}{2} .
\end{array}\right.
$$

Since $\varphi\left(\frac{1}{2}\right)=\varphi^{\prime}\left(\frac{1}{2}\right)=0$ and $\varphi \in C^{\infty}\left(\left(\frac{1}{2}, \frac{3}{2}\right)\right)$, then we have $\frac{\partial u_{k}}{\partial x} \in C^{0}\left(\overline{\mathcal{B}_{1}^{z_{0}}}\right)$. Moreover, we obtain:

$$
\begin{aligned}
\frac{1}{3} \int_{A_{1 / 2,1}}\left|\frac{\partial u_{k}}{\partial x}\right|^{2}( & x, y, z) \mathrm{d}(x, y) \leq \int_{A_{1 / 2,1}}\left|\frac{2 x y}{\left(\sqrt{x^{2}+y^{2}}\right)^{3}}\right|^{2}\left|\varphi\left(\sqrt{x^{2}+y^{2}}\right)\right|^{2}\left|\frac{\partial Q_{k}}{\partial y^{\prime}}\right|^{2}\left(x^{\prime}, y^{\prime}, z\right) \mathrm{d}(x, y) \\
& +\int_{A_{1 / 2,1}}\left|\frac{2 y^{2}}{\left(\sqrt{x^{2}+y^{2}}\right)^{3}}-1\right|^{2}\left|\varphi\left(\sqrt{x^{2}+y^{2}}\right)\right|^{2}\left|\frac{\partial Q_{k}}{\partial x^{\prime}}\right|^{2}\left(x^{\prime}, y^{\prime}, z\right) \mathrm{d}(x, y) \\
& +\int_{A_{1 / 2,1}}\left|\frac{x}{\sqrt{x^{2}+y^{2}}}\right|^{2}\left|\varphi^{\prime}\left(\sqrt{x^{2}+y^{2}}\right)\right|^{2}\left|Q_{k}\left(x^{\prime}, y^{\prime}, z\right)-f_{A_{1,3 / 2}} Q_{k}(s, t, z) \mathrm{d}(s, t)\right|^{2} \mathrm{~d}(x, y) .
\end{aligned}
$$

By the definition of $\varphi$, we have $\|\varphi\|_{L^{\infty}(\mathbb{R})}=1$ and $\left\|\varphi^{\prime}\right\|_{L^{\infty}(\mathbb{R})}=2 \sqrt{9+6 \sqrt{3}} \cdot e^{1-\sqrt{3}} \approx 4.23<5$ (the maximum is obtained for $\rho=1-\frac{1}{6} \sqrt{6 \sqrt{3}-9}$ ).

For any $(x, y)$ such that $\frac{1}{2} \leq \sqrt{x^{2}+y^{2}} \leq 1$, we have:

$$
\begin{aligned}
& -\left|\frac{x}{\sqrt{x^{2}+y^{2}}}\right|^{2}=\frac{x^{2}}{x^{2}+y^{2}} \leq 1 ; \\
& -\left|\frac{2 x y}{x^{2}+y^{2}}\right| \leq 1 \Rightarrow\left|\frac{2 x y}{\left(\sqrt{x^{2}+y^{2}}\right)^{3}}\right|^{2} \leq \frac{1}{x^{2}+y^{2}} \leq 2 ; \\
& -0 \leq \frac{2 y^{2}}{\left(\sqrt{x^{2}+y^{2}}\right)^{3}} \leq \frac{2\left(x^{2}+y^{2}\right)}{\left(\sqrt{x^{2}+y^{2}}\right)^{3}} \leq 4 \Rightarrow-1 \leq \frac{2 y^{2}}{\left(\sqrt{x^{2}+y^{2}}\right)^{3}}-1 \leq 3 \Rightarrow\left|\frac{2 y^{2}}{\left(\sqrt{x^{2}+y^{2}}\right)^{3}}\right|^{2} \leq 9 .
\end{aligned}
$$

Therefore

$$
\begin{aligned}
\frac{1}{3} \int_{A_{1 / 2,1}}\left|\frac{\partial u_{k}}{\partial x}\right|^{2}(x, y, z) \mathrm{d}(x, y) \leq & 25 \int_{A_{1 / 2,1}}\left|Q_{k}\left(x^{\prime}, y^{\prime}, z\right)-f_{A_{1,3 / 2}} Q_{k}(s, t, z) \mathrm{d}(s, t)\right|^{2} \mathrm{~d}(x, y) \\
& +9 \int_{A_{1 / 2,1}}\left|\frac{\partial Q_{k}}{\partial x^{\prime}}\right|^{2}\left(x^{\prime}, y^{\prime}, z\right) \mathrm{d}(x, y)+4 \int_{A_{1 / 2,1}}\left|\frac{\partial Q_{k}}{\partial y^{\prime}}\right|^{2}\left(x^{\prime}, y^{\prime}, z\right) \mathrm{d}(x, y) .
\end{aligned}
$$

Using now the change of variables $\left(x^{\prime}, y^{\prime}\right)=T(x, y)$, we obtain

$$
\mathrm{d}(x, y)=\left(\frac{2}{\sqrt{\left(x^{\prime}\right)^{2}+\left(y^{\prime}\right) 2}}-1\right) \mathrm{d}\left(x^{\prime}, y^{\prime}\right)
$$


and since $\left(x^{\prime}, y^{\prime}\right) \in A_{1,3 / 2}$, we get $1 \geq \frac{2}{\sqrt{\left(x^{\prime}\right)^{2}+\left(y^{\prime}\right)^{2}}}-1 \geq \frac{1}{3}$, which implies

$$
\begin{aligned}
\int_{A_{1 / 2,1}}\left|\frac{\partial u_{k}}{\partial x}\right|^{2}(x, y, z) \mathrm{d}(x, y) \leq & 75 \int_{A_{1,3 / 2}}\left|Q_{k}\left(x^{\prime}, y^{\prime}, z\right)-f_{A_{1,3 / 2}} Q_{k}(s, t, z) \mathrm{d}(s, t)\right|^{2} \mathrm{~d}\left(x^{\prime}, y^{\prime}\right) \\
& +27 \int_{A_{1,3 / 2}}\left|\frac{\partial Q_{k}}{\partial x^{\prime}}\right|^{2}\left(x^{\prime}, y^{\prime}, z\right) \mathrm{d}\left(x^{\prime}, y^{\prime}\right)+12 \int_{A_{1,3 / 2}}\left|\frac{\partial Q_{k}}{\partial y^{\prime}}\right|^{2}\left(x^{\prime}, y^{\prime}, z\right) \mathrm{d}\left(x^{\prime}, y^{\prime}\right) .
\end{aligned}
$$

For the first term from the right hand side from the last inequality we can apply the Poincaré inequality, since $Q_{k}(\cdot, \cdot, z) \in H^{1}\left(A_{1,3 / 2}\right)$, for any $z \in\left(-z_{0}, z_{0}\right)$. Therefore

$$
\begin{aligned}
\int_{A_{1 / 2,1}}\left|\frac{\partial u_{k}}{\partial x}\right|^{2}(x, y, z) \mathrm{d}(x, y) \leq & 75 \cdot C_{P}\left(A_{1,3 / 2}\right) \int_{A_{1,3 / 2}}\left(\left|\frac{\partial Q_{k}}{\partial x^{\prime}}\right|^{2}\left(x^{\prime}, y^{\prime}, z\right)+\left|\frac{\partial Q_{k}}{\partial y^{\prime}}\right|^{2}\left(x^{\prime}, y^{\prime}, z\right)\right) \mathrm{d}\left(x^{\prime}, y^{\prime}\right) \\
& +27 \int_{A_{1,3 / 2}}\left|\frac{\partial Q_{k}}{\partial x^{\prime}}\right|^{2}\left(x^{\prime}, y^{\prime}, z\right) \mathrm{d}(x, y)+12 \int_{A_{1,3 / 2}}\left|\frac{\partial Q_{k}}{\partial y^{\prime}}\right|^{2}\left(x^{\prime}, y^{\prime}, z\right) \mathrm{d}(x, y),
\end{aligned}
$$

where $C_{P}\left(A_{1,3 / 2}\right)$ is the Poincaré constant for the two dimmensional domain $A_{1,3 / 2}$. Hence, there exists $c_{1}>0$, independent of $z_{0}$, such that:

$$
\int_{A_{1 / 2,1}}\left|\frac{\partial u_{k}}{\partial x}\right|^{2}(x, y, z) \mathrm{d}(x, y, z) \leq c_{1} \int_{A_{1,3 / 2}}\left(\left|\frac{\partial Q_{k}}{\partial x^{\prime}}\right|^{2}\left(x^{\prime}, y^{\prime}, z\right)+\left|\frac{\partial Q_{k}}{\partial y^{\prime}}\right|^{2}\left(x^{\prime}, y^{\prime}, z\right)\right) \mathrm{d}\left(x^{\prime}, y^{\prime}, z\right) .
$$

Integrating now with respect to $z \in\left(-z_{0}, z_{0}\right)$, we get:

$$
\begin{aligned}
\int_{\mathcal{A}_{1 / 2,1}^{z_{0}}}\left|\frac{\partial u_{k}}{\partial x}\right|^{2}(x, y, z) \mathrm{d}(x, y, z) & \leq c_{1} \int_{\mathcal{A}_{1,3 / 2}^{z_{0}}}\left(\left|\frac{\partial Q_{k}}{\partial x^{\prime}}\right|^{2}\left(x^{\prime}, y^{\prime}, z\right)+\left|\frac{\partial Q_{k}}{\partial y^{\prime}}\right|^{2}\left(x^{\prime}, y^{\prime}, z\right)\right) \mathrm{d}\left(x^{\prime}, y^{\prime}, z\right) \\
\Rightarrow\left\|\frac{\partial u_{k}}{\partial x}\right\|_{L^{2}\left(\mathcal{A}_{1 / 2,1}^{z_{0}}\right)}^{2} & \leq c_{1}\left\|\nabla Q_{k}\right\|_{L^{2}\left(\mathcal{A}_{1,3 / 2}^{z_{0}}\right)}^{2} .
\end{aligned}
$$

Using now the fact that $\frac{\partial u_{k}}{\partial x} \in C^{0}\left(\overline{\mathcal{B}_{1}^{z_{0}}}\right)$ and that $\frac{\partial u_{k}}{\partial x}(x, y, z)=0$ if $0 \leq \sqrt{x^{2}+y^{2}} \leq \frac{1}{2}$, then we can write:

$$
\left\|\frac{\partial u_{k}}{\partial x}\right\|_{L^{2}\left(\mathcal{B}_{1}^{\left.z_{0}\right)}\right.}^{2} \leq c_{1}\left\|\nabla Q_{k}\right\|_{L^{2}\left(\mathcal{A}_{1,3 / 2}^{z_{0}}\right)}^{2} .
$$


In a similar fashion, $\frac{\partial u_{k}}{\partial y}, \frac{\partial u_{k}}{\partial z} \in C^{0}\left(\overline{\mathcal{B}_{1}^{z_{0}}}\right)$, where

$$
\frac{\partial u_{k}}{\partial y}(x, y, z)=\left\{\begin{array}{l}
\frac{y}{\sqrt{x^{2}+y^{2}}} \cdot \varphi^{\prime}\left(\sqrt{x^{2}+y^{2}}\right) \cdot\left(Q_{k}\left(x^{\prime}, y^{\prime}, z\right)-f_{A_{1,3 / 2}} Q_{k}(s, t, z) \mathrm{d}(s, t)\right) \\
\quad-\frac{2 x y}{\left(\sqrt{x^{2}+y^{2}}\right)^{3}} \cdot \varphi\left(\sqrt{x^{2}+y^{2}}\right) \cdot\left(\frac{\partial Q_{k}}{\partial x^{\prime}}\left(x^{\prime}, y^{\prime}, z\right)\right) \\
\quad+\left(\frac{2 x^{2}}{\left(\sqrt{\left.x^{2}+y^{2}\right)}\right)^{3}}-1\right) \cdot \varphi\left(\sqrt{x^{2}+y^{2}}\right) \cdot\left(\frac{\partial Q_{k}}{\partial y^{\prime}}\left(x^{\prime}, y^{\prime}, z\right)\right), \text { for } \frac{1}{2} \leq \sqrt{x^{2}+y^{2}} \leq 1 \\
0, \text { for } 0 \leq \sqrt{x^{2}+y^{2}} \leq \frac{1}{2}
\end{array}\right.
$$

with

$$
\left\|\frac{\partial u_{k}}{\partial y}\right\|_{L^{2}\left(\mathcal{B}_{1}^{z_{0}}\right)}^{2} \leq c_{1}\left\|\nabla Q_{k}\right\|_{L^{2}\left(\mathcal{A}_{1,3 / 2}^{z_{0}}\right)}^{2} .
$$

and

$$
\frac{\partial u_{k}}{\partial z}(x, y, z)=\left\{\begin{array}{l}
\varphi\left(\sqrt{x^{2}+y^{2}}\right) \frac{\partial Q_{k}}{\partial z}\left(x^{\prime}, y^{\prime}, z\right) \\
+\left(1-\varphi\left(\sqrt{x^{2}+y^{2}}\right)\right) f_{A_{1,3 / 2}} \frac{\partial Q_{k}}{\partial z}(s, t, z) \mathrm{d}(s, t), \text { for } \frac{1}{2} \leq \sqrt{x^{2}+y^{2}} \leq 1 \\
f_{A_{1,3 / 2}} \frac{\partial Q_{k}}{\partial z}(s, t, z) d(s, t), \text { for } 0 \leq \sqrt{x^{2}+y^{2}} \leq \frac{1}{2}
\end{array}\right.
$$

since $Q \in C^{\infty}\left(\overline{\mathcal{A}_{1,3 / 2}^{z_{0}}}\right)$ and $A_{1,3 / 2}$ is independent of $z$, so we can move the derivative under the integral.

Then for any $(x, y, z) \in \mathcal{A}_{1 / 2,1}^{z_{0}}$, we have:

$$
\begin{aligned}
\frac{1}{2} \int_{A_{1 / 2,1}}\left|\frac{\partial u_{k}}{\partial z}\right|^{2}(x, y, z) \mathrm{d}(x, y) \leq & \|\varphi\|_{L^{\infty}(\mathbb{R})}^{2} \int_{A_{1 / 2,1}}\left|\frac{\partial Q_{k}}{\partial z}\right|^{2}\left(x^{\prime}, y^{\prime}, z\right) \mathrm{d}(x, y) \\
& +\|1-\varphi\|_{L^{\infty}(\mathbb{R})}^{2} \int_{A_{1 / 2,1}}\left|f_{A_{1,3 / 2}} \frac{\partial Q_{k}}{\partial z}(s, t, z) \mathrm{d}(s, t)\right|^{2} \mathrm{~d}(x, y) \\
& \leq \int_{A_{1,3 / 2}}\left|\frac{\partial Q_{k}}{\partial z}\right|^{2}\left(x^{\prime}, y^{\prime}, z\right) \mathrm{d}\left(x^{\prime}, y^{\prime}\right)+\frac{3 \pi}{4} \cdot \frac{16}{25 \pi^{2}} \int_{A_{1,3 / 2}}\left|\frac{\partial Q_{k}}{\partial z}\right|^{2}\left(x^{\prime}, y^{\prime}, z\right) \mathrm{d}\left(x^{\prime}, y^{\prime}\right)
\end{aligned}
$$

and integrating with respect to $z \in\left(-z_{0}, z_{0}\right)$, we obtain

$$
\int_{\mathcal{A}_{1 / 2,1}^{z_{0}}}\left|\frac{\partial u_{k}}{\partial z}\right|^{2}(x, y, z) \mathrm{d}(x, y, z) \leq\left(2+\frac{24}{25 \pi}\right) \int_{\mathcal{A}_{1,3 / 2}^{z_{0}}}\left|\frac{\partial Q_{k}}{\partial z}\right|^{2}\left(x^{\prime}, y^{\prime}, z\right) \mathrm{d}\left(x^{\prime}, y^{\prime}, z\right) .
$$

For any $(x, y, z) \in \mathcal{B}_{1}^{z_{0}}$ with $0 \leq \sqrt{x^{2}+y^{2}} \leq \frac{1}{2}$ we have:

$$
\left|\frac{\partial u_{k}}{\partial z}\right|^{2}(x, y, z) \leq \frac{16}{25 \pi^{2}} \int_{A_{1,3 / 2}}\left|\frac{\partial Q_{k}}{\partial z}\right|^{2}\left(x^{\prime}, y^{\prime}, z\right) \mathrm{d}\left(x^{\prime}, y^{\prime}\right)
$$


which implies

$$
\int_{\overline{B_{1 / 2}}}\left|\frac{\partial u_{k}}{\partial z}\right|^{2}(x, y, z) \mathrm{d}(x, y) \leq \frac{4}{25 \pi} \int_{A_{1,3 / 2}}\left|\frac{\partial Q_{k}}{\partial z}\right|^{2}\left(x^{\prime}, y^{\prime}, z\right) \mathrm{d}\left(x^{\prime}, y^{\prime}, z\right)
$$

and from here we obtain that

$$
\left\|\frac{\partial u_{k}}{\partial z}\right\|_{L^{2}\left(\mathcal{B}_{1}^{\left.z_{0}\right)}\right.}^{2} \leq\left(3+\frac{3}{25 \pi}\right)\left\|\nabla Q_{k}\right\|_{L^{2}\left(\mathcal{A}_{1,3 / 2}^{z_{0}}\right)}^{2} .
$$

Now we prove that we can control $\left\|u_{k}\right\|_{L^{2}\left(\mathcal{B}_{1}^{z_{0}}\right)}$ with $\left\|Q_{k}\right\|_{L^{2}\left(\mathcal{A}_{1,3 / 2}^{z_{0}}\right)}$. For any $(x, y) \in A_{1 / 2,1}$, we have:

$$
\left|u_{k}\right|^{2}(x, y, z) \leq \mid \varphi\left(\left.\sqrt{x^{2}+y^{2}}\right|^{2}\left|Q_{k}\left(x^{\prime}, y^{\prime}, z\right)\right|^{2}+\left(1-\varphi\left(\sqrt{x^{2}+y^{2}}\right)\right)^{2}\left|f_{A_{1,3 / 2}} Q_{k}(s, t, z) \mathrm{d}(s, t)\right|^{2}\right.
$$

which implies

$$
\begin{aligned}
\frac{1}{2} \int_{A_{1 / 2,1}}\left|u_{k}\right|^{2}(x, y, z) \mathrm{d}(x, y) \leq & \|1-\varphi\|_{L^{\infty}(\mathbb{R})}^{2} \cdot \frac{3}{4 \pi} \cdot \frac{16}{25 \pi^{2}} \int_{A_{1,3 / 2}}\left|Q_{k}\right|^{2}\left(x^{\prime}, y^{\prime}, z\right) \mathrm{d}(x, y) \\
& +\|\varphi\|_{L^{\infty}(\mathbb{R})}^{2} \int_{A_{1 / 2,1}}\left|Q_{k}\right|^{2}\left(x^{\prime}, y^{\prime}, z\right) \mathrm{d}(x, y) .
\end{aligned}
$$

Using the same change of variables, the same bounds for $\varphi$ and for $1-\varphi$ and integrating with respect to $z \in\left(-z_{0}, z_{0}\right)$, we get:

$$
\left\|u_{k}\right\|_{L^{2}\left(\mathcal{A}_{1 / 2,1}^{\left.z_{0}\right)}\right.}^{2} \leq\left(2+\frac{24}{25 \pi}\right)\left\|Q_{k}\right\|_{L^{2}\left(\mathcal{A}_{1,3 / 2}^{z_{0}}\right)}^{2} .
$$

For any $(x, y) \in \overline{B_{1 / 2}}$ we have:

$$
\left|u_{k}\right|^{2}(x, y, z)=\left|f_{A_{1,3 / 2}} Q_{k}(s, t, z) \mathrm{d}(s, t)\right|^{2}
$$

which implies

$$
\left\|u_{k}\right\|_{L^{2}\left(\overline{B_{1 / 2}} \times\left(-z_{0}, z_{0}\right)\right)}^{2} \leq \frac{4}{25 \pi}\left\|Q_{k}\right\|_{L^{2}\left(\mathcal{A}_{1,3 / 2}^{z_{0}}\right)}^{2}
$$

hence

$$
\left\|u_{k}\right\|_{L^{2}\left(\mathcal{B}_{1}^{z_{0}}\right)}^{2} \leq\left(3+\frac{3}{25 \pi}\right)\left\|Q_{k}\right\|_{L^{2}\left(\mathcal{A}_{1,3 / 2}^{z_{0}}\right)}^{2} .
$$

Combining all the relations that we have obtained, we see that for any $k \geq 1$ we have:

$-u_{k} \in H^{1}\left(\mathcal{B}_{1}^{z_{0}}\right)$;

- $\left\|u_{k}\right\|_{L^{2}\left(\mathcal{B}_{1}^{z_{0}}\right)} \leq c_{2}\left\|Q_{k}\right\|_{L^{2}\left(\mathcal{A}_{1,3 / 2}^{z_{0}}\right)}$, where $c_{2}=\sqrt{3+\frac{3}{25 \pi}} ;$ 
$-\left\|\nabla u_{k}\right\|_{L^{2}\left(\mathcal{B}_{1}^{\left.z_{0}\right)}\right.} \leq c_{3}\left\|\nabla Q_{k}\right\|_{L^{2}\left(\mathcal{A}_{1,3 / 2}^{z_{0}}\right)}$, where $c_{3}=\max \left\{\sqrt{c_{1}}, c_{2}\right\}$.

Now, if we repeat the same argument (as the one used in order to achieve the $L^{2}$ control between $u_{k}$ and $Q_{k}$ ) for the functions $\left(u_{k}-u\right)$, for any $k \geq 1$, we get:

$$
\left\|u_{k}-u\right\|_{L^{2}\left(\mathcal{B}_{1}^{\left.z_{0}\right)}\right.} \leq c_{2}\left\|Q_{k}-Q\right\|_{L^{2}\left(\mathcal{A}_{1,3 / 2}^{z_{0}}\right)}
$$

and since $Q_{k} \rightarrow Q$ strongly in $H^{1}\left(\mathcal{A}_{1,3 / 2}^{z_{0}}\right)$, hence in $L^{2}\left(\mathcal{A}_{1,3 / 2}^{z_{0}}\right)$, we obtain that $u_{k} \rightarrow u$ strongly in $L^{2}\left(\mathcal{B}_{1}^{z_{0}}\right)$.

Because $Q_{k} \rightarrow Q$ strongly in $H^{1}\left(\mathcal{A}_{1,3 / 2}^{z_{0}}\right)$, then $\left(Q_{k}\right)_{k \geq 1}$ is a bounded sequence in $H^{1}\left(\mathcal{A}_{1,3 / 2}^{z_{0}}\right)$ and using the inequalities proved before, we get that $\left(u_{k}\right)_{k \geq 1}$ is a bounded sequence in $H^{1}\left(\mathcal{B}_{1}^{z_{0}}\right)$, therefore there exists a subsequence $\left(u_{k_{j}}\right)_{j \geq 1}$ which has the property that $u_{k_{j}} \rightarrow u_{0}$, with $u_{0} \in H^{1}\left(\mathcal{B}_{1}^{z_{0}}\right)$. From here, we have the following convergences in $L^{2}\left(\mathcal{B}_{1}^{z_{0}}\right): u_{k_{j}} \rightarrow u_{0}$ and $u_{k_{j}} \rightarrow u$, so $u=u_{0}$ a.e. in $\mathcal{B}_{1}^{z_{0}}$. However, since $u_{0} \in H^{1}\left(\mathcal{B}_{1}^{z_{0}}\right)$, we obtain that $u \in H^{1}\left(\mathcal{B}_{1}^{z_{0}}\right)$ with $\nabla u=\nabla u_{0}$ a.e. in $\mathcal{B}_{1}^{z_{0}}$.

Let $\tilde{u}_{x}: \mathcal{B}_{1}^{z_{0}} \rightarrow \mathbb{R}$ be the function defined as:

$$
\tilde{u}_{x}(x, y, z)=\left\{\begin{array}{l}
\frac{x}{\sqrt{x^{2}+y^{2}}} \cdot \varphi^{\prime}\left(\sqrt{x^{2}+y^{2}}\right) \cdot\left(Q\left(x^{\prime}, y^{\prime}, z\right)-f_{A_{1,3 / 2}} Q(s, t, z) \mathrm{d}(s, t)\right) \\
+\left(\frac{2 y^{2}}{\left.\left(\sqrt{x^{2}+y^{2}}\right)\right)^{3}}-1\right) \cdot \varphi\left(\sqrt{x^{2}+y^{2}}\right) \cdot\left(\frac{\partial Q}{\partial x^{\prime}}\left(x^{\prime}, y^{\prime}, z\right)\right) \\
\quad-\frac{2 x y}{\left(\sqrt{x^{2}+y^{2}}\right)^{3}} \cdot \varphi\left(\sqrt{x^{2}+y^{2}}\right) \cdot\left(\frac{\partial Q}{\partial y^{\prime}}\left(x^{\prime}, y^{\prime}, z\right)\right), \text { for } \frac{1}{2} \leq \sqrt{x^{2}+y^{2}}<1 \\
0, \text { for } 0 \leq \sqrt{x^{2}+y^{2}} \leq \frac{1}{2} .
\end{array}\right.
$$

for every $z \in\left(-z_{0}, z_{0}\right)$.

Using the same argument as before (we only control the $L^{2}$ norm), we can see that:

$$
\left\|\frac{\partial u_{k}}{\partial x}-\tilde{u}_{x}\right\|_{L^{2}\left(\mathcal{B}_{1}^{z_{0}}\right)} \leq c_{3}\left\|\nabla Q_{k}-\nabla Q\right\|_{L^{2}\left(\mathcal{A}_{1,3 / 2}^{z_{0}}\right)}
$$

and since $\nabla Q_{k} \rightarrow \nabla Q$ strongly in $L^{2}\left(\mathcal{A}_{1,3 / 2}^{z_{0}}\right)$, we obtain that $\frac{\partial u_{k}}{\partial x} \rightarrow \tilde{u}_{x}$ strongly in $L^{2}\left(\mathcal{B}_{1}^{z_{0}}\right)$. But at the same time, we have $\frac{\partial u_{k}}{\partial x} \rightarrow \frac{\partial u}{\partial x}$ weakly in $L^{2}\left(\mathcal{B}_{1}^{z_{0}}\right)$, hence $\frac{\partial u}{\partial x}=\tilde{u}_{x}$ a.e. in $L^{2}\left(\mathcal{B}_{1}^{z_{0}}\right)$ and $\frac{\partial u_{k}}{\partial x} \rightarrow \frac{\partial u}{\partial x}$ strongly in $L^{2}\left(\mathcal{B}_{1}^{z_{0}}\right)$. Applying the same argument, we finally prove that $\nabla u_{k} \rightarrow \nabla u$ strongly in $L^{2}\left(\mathcal{B}_{1}^{z_{0}}\right)$.

In the end, we see that:

$$
\begin{aligned}
\|\nabla u\|_{L^{2}\left(\mathcal{B}_{1}^{z_{0}}\right)} & \leq\left\|\nabla u-\nabla u_{k}\right\|_{L^{2}\left(\mathcal{B}_{1}^{z_{0}}\right)}+\left\|\nabla u_{k}\right\|_{L^{2}\left(\mathcal{B}_{1}^{z_{0}}\right)} \\
& \leq\left\|\nabla u-\nabla u_{k}\right\|_{L^{2}\left(\mathcal{B}_{1}^{z_{0}}\right)}+c_{3}\left\|\nabla Q_{k}\right\|_{L^{2}\left(\mathcal{A}_{1,3 / 2}^{z_{0}}\right)} \\
& \leq\left\|\nabla u-\nabla u_{k}\right\|_{L^{2}\left(\mathcal{B}_{1}^{z_{0}}\right)}+c_{3}\left\|\nabla Q_{k}-\nabla Q\right\|_{L^{2}\left(\mathcal{A}_{1,3 / 2}^{z_{0}}\right)}+c_{3}\|\nabla Q\|_{L^{2}\left(\mathcal{A}_{1,3 / 2}^{z_{0}}\right)} .
\end{aligned}
$$

Because $\nabla u_{k} \rightarrow \nabla u$ strongly in $L^{2}\left(\mathcal{B}_{1}^{z_{0}}\right)$ and because $\nabla Q_{k} \rightarrow \nabla Q$ strongly in $L^{2}\left(\mathcal{A}_{1,3 / 2}^{z_{0}}\right)$, we conclude that

$$
\|\nabla u\|_{L^{2}\left(\mathcal{B}_{1}^{\left.z_{0}\right)}\right.} \leq c_{3}\|\nabla Q\|_{L^{2}\left(\mathcal{A}_{1,3 / 2}^{z_{0}}\right)} .
$$


Now we transform in several steps the sets $\mathcal{B}_{1}^{z_{0}}$ and $\mathcal{A}_{1,3 / 2}^{z_{0}}$ from the previous lemma into the corresponding regions related to $\Omega_{\varepsilon}$ and $\mathcal{N}_{\varepsilon}^{\mathcal{T}}$, that is, $\mathcal{B}_{1}^{z_{0}}$ into $\mathcal{P}_{\varepsilon}^{z, m}$, which is included in $\mathcal{N}_{\varepsilon}^{\mathcal{T}}$, and $\mathcal{A}_{1,3 / 2}^{z_{0}}$ into a parallelepiped with an interior hole, surrounding $\mathcal{P}_{\varepsilon}^{z, m}$, which is included in $\Omega_{\varepsilon}$ (the hole is exactly the "connecting parallelepiped" $\left.\mathcal{P}_{\varepsilon}^{z, m}\right)$.

Let $T_{2}: \mathbb{R}^{3} \rightarrow \mathbb{R}^{3}$ be the transformation defined as:

$$
T_{2}(x, y, z)= \begin{cases}(0,0, z) & \text { if } x=y=0, \\ \left(\sqrt{x^{2}+y^{2}}, \frac{4}{\pi} \sqrt{x^{2}+y^{2}} \arctan \frac{y}{x}, z\right) & \text { if }|y| \leq x, x>0, \\ \left.-\sqrt{x^{2}+y^{2}},-\frac{4}{\pi} \sqrt{x^{2}+y^{2}} \arctan \frac{y}{x}, z\right) & \text { if }|y| \leq-x, x<0, \\ \left(\frac{4}{\pi} \sqrt{x^{2}+y^{2}} \arctan \frac{x}{y}, \sqrt{x^{2}+y^{2}}, z\right) & \text { if }|x| \leq y, y>0, \\ \left(-\frac{4}{\pi} \sqrt{x^{2}+y^{2}} \arctan \frac{x}{y},-\sqrt{x^{2}+y^{2}}, z\right) & \text { if }|x| \leq-y, y<0,\end{cases}
$$

with the inverse

$$
T_{2}^{-1}(\xi, \eta, z)= \begin{cases}(0,0, z) & \text { if } \eta=\xi=0, \\ \left(\xi \cos \frac{\pi}{4} \frac{\eta}{\xi}, \xi \sin \frac{\pi}{4} \frac{\eta}{\xi}, z\right) & \text { if }|\eta| \leq|\xi|, \xi \neq 0, \\ \left(\eta \sin \frac{\pi}{4} \frac{\xi}{\eta}, \eta \cos \frac{\pi}{4} \frac{\xi}{\eta}, z\right) & \text { if }|\xi| \leq|\eta|, \eta \neq 0 .\end{cases}
$$

More specifically, $T_{2}(x, y, z)=\left(\Lambda_{2}(x, y), z\right)$, where $\Lambda_{2}$ is, according to [17], a bi-Lipschitz continuous map that maps, in $\mathbb{R}^{2}$, the unit ball into the unit cube and the Jacobian of $\Lambda_{2}$ is constant almost everywhere in $\mathbb{R}^{2}$. Hence, the transformation $T_{2}$ is bi-Lipschitz and the Jacobian of $T_{2}$ is constant almost everywhere in $\mathbb{R}^{3}$.

In our case, we have: $T_{2}\left(\mathcal{B}_{1}^{z_{0}}\right)=(-1,1)^{2} \times\left(-z_{0}, z_{0}\right)$ and $T_{2}\left(\mathcal{A}_{1,3 / 2}^{z_{0}}\right)=\left((-3 / 2,3 / 2)^{2} \backslash(-1,1)^{2}\right) \times\left(-z_{0}, z_{0}\right)$.

Let $u \in H^{1}\left(\mathcal{B}_{1}^{z_{0}}\right), Q \in H^{1}\left(\mathcal{A}_{1,3 / 2}^{z_{0}}\right)$ and the constant $c>0$ such that $\|\nabla u\|_{L^{2}\left(\mathcal{B}_{1}^{z_{0}}\right)} \leq c\|\nabla Q\|_{L^{2}\left(\mathcal{A}_{1,3 / 2}^{z_{0}}\right)}$ be given the previous lemma, constant which is independent of $z_{0}$. Then we obtain that the functions $\tilde{u}:=u \circ T_{2}^{-1} \in$ $H^{1}\left((-1,1)^{2} \times\left(-z_{0}, z_{0}\right)\right)$ and $\tilde{Q}:=Q \circ T_{2}^{-1} \in H^{1}\left(\left((-3 / 2,3 / 2)^{2} \backslash(-1,1)^{2}\right) \times\left(-z_{0}, z_{0}\right)\right)$ and that there exists constants $c_{j}$ and $c_{J}$, which are also independent of $z_{0}$, but dependent on the constants given by the Jacobians of $T_{2}$ and $T_{2}^{-1}$, such that:

$$
c_{j}\|\nabla \tilde{u}\|_{L^{2}\left(T_{2}\left(\mathcal{B}_{1}^{z_{0}}\right)\right)}^{2} \leq\|\nabla u\|_{L^{2}\left(\mathcal{B}_{1}^{z_{0}}\right)}^{2} \leq c_{J}\|\nabla \tilde{u}\|_{L^{2}\left(T_{2}\left(\mathcal{B}_{1}^{z_{0}}\right)\right)}^{2}
$$

and

$$
c_{j}\|\nabla \tilde{Q}\|_{L^{2}\left(T_{2}\left(\mathcal{A}_{1,3 / 2}^{z_{0}}\right)\right)}^{2} \leq\|\nabla Q\|_{L^{2}\left(\mathcal{A}_{1,3 / 2}^{z_{0}}\right)}^{2} \leq c_{J}\|\nabla \tilde{Q}\|_{L^{2}\left(T_{2}\left(\mathcal{A}_{1,3 / 2}^{z_{0}}\right)\right)}^{2} .
$$

Hence, the inequality $\|\nabla u\|_{L^{2}\left(\mathcal{B}_{1}^{\left.z_{0}\right)}\right.} \leq c\|\nabla Q\|_{L^{2}\left(\mathcal{A}_{1,3 / 2}^{\left.z_{0}\right)}\right.}$ implies that there exists a constant $c_{0}$, also independent of $z_{0}$, such that:

$$
\|\nabla \tilde{u}\|_{L^{2}\left(T_{2}\left(\mathcal{B}_{1}^{z_{0}}\right)\right)} \leq c_{0}\|\nabla \tilde{Q}\|_{L^{2}\left(T_{2}\left(\mathcal{A}_{1,3 / 2}^{z_{0}}\right)\right)}
$$


Now if we use the transformation $T_{3}(x, y, z)=\varepsilon^{\alpha}(x, y, z)$ and denote $\bar{u}:=\tilde{u} \circ T_{3}^{-1}$ and $\bar{Q}:=\tilde{Q} \circ T_{3}^{-1}$, we get:

$$
\varepsilon^{-\alpha}\|\nabla \bar{u}\|_{L^{2}\left(\left(T_{3} \circ T_{2}\right)\left(\mathcal{B}_{1}^{z_{0}}\right)\right)}^{2}=\|\nabla \tilde{u}\|_{L^{2}\left(T_{2}\left(\mathcal{B}_{1}^{z_{0}}\right)\right)}^{2} \leq c_{0}^{2}\|\nabla \tilde{Q}\|_{L^{2}\left(T_{2}\left(\mathcal{A}_{1,3 / 2}^{z_{0}}\right)\right)}^{2}=c_{0}^{2} \varepsilon^{-\alpha}\|\nabla \bar{Q}\|_{L^{2}\left(\left(T_{3} \circ T_{2}\right)\left(\mathcal{B}_{1}^{z_{0}}\right)\right)}^{2}
$$

which implies that

$$
\|\nabla \bar{u}\|_{L^{2}\left(\left(T_{3} \circ T_{2}\right)\left(\mathcal{B}_{1}^{z_{0}}\right)\right)} \leq c_{0}\|\nabla \bar{Q}\|_{L^{2}\left(\left(T_{3} \circ T_{2}\right)\left(\mathcal{A}_{1,3 / 2}^{z_{0}}\right)\right)}
$$

Since the constant $c_{0}$ is independent of the choice of $z_{0}$, we can have $z_{0}=\frac{r \varepsilon-\varepsilon^{\alpha}}{\varepsilon^{\alpha}}$.

The final change of variables is based on the mapping $T_{4}: \mathbb{R}^{3} \rightarrow \mathbb{R}^{3}$ defined as: $T_{4}(x, y, z)=\left(\frac{x}{2 p}, \frac{y}{2 q}, \frac{z}{2 r}\right)$, where $p, q$ and $r$ are from relation (2.4). In this way, if we translate the origin into the center of the parallelepiped $\mathcal{P}_{\varepsilon}^{z, m}$, we obtain that $\left(T_{4} \circ T_{3} \circ T_{2}\right)\left(\mathcal{B}_{1}^{z_{0}}\right)=\mathcal{P}_{\varepsilon}^{z, m}$ and we denote by $\mathcal{R}_{\varepsilon}^{z, m}$ the set $\left(T_{4} \circ T_{3} \circ T_{2}\right)\left(\mathcal{A}_{1,3 / 2}^{z_{0}}\right)$, which is the box contained in $\Omega_{\varepsilon}$ (for $\varepsilon$ small enough) that "surrounds" $\mathcal{P}_{\varepsilon}^{z, m}$.

The transformation $T_{4}$ is bi-Lipschitz and applying the same arguments as before, we obtain that there exists a function $u \in H^{1}\left(\mathcal{P}_{\varepsilon}^{z, m}\right)$ ( $u$ can be seen as $\bar{u} \circ\left(T_{4}^{-1}\right)$ ) such that $u=Q$ on the "contact" faces $\mathcal{T}_{z}^{m}$ of $\mathcal{P}_{\varepsilon}^{z, m}$ and an $\varepsilon$-independent constant $c>0$ such that

$$
\|\nabla u\|_{L^{2}\left(\mathcal{P}_{\varepsilon}^{z, m}\right)} \leq c\|\nabla Q\|_{L^{2}\left(\mathcal{R}_{\varepsilon}^{z, m}\right)}
$$

Since the objects $\mathcal{R}_{\varepsilon}^{z, m}$ are pairwise disjoint (if we look only at the boxes surrounding the "connecting parallelepipeds" with centers in $\mathcal{Y}_{\varepsilon}^{z}$ ), repeating the same argument for every other "connecting parallelepiped" of this type (with centers in $\mathcal{Y}_{\varepsilon}^{z}$ ) and then repeating the same argument for all the others "connecting parallelepipeds" from $\mathcal{N}_{\varepsilon}^{\mathcal{T}}$ (that is, with centers in $\left.\mathcal{Y}_{\varepsilon}\right)$, we obtain $u \in H^{1}\left(\Omega_{\varepsilon} \cup \mathcal{N}_{\varepsilon}^{\mathcal{T}}, \mathcal{S}_{0}\right)$ an extension of $Q \in H^{1}\left(\Omega_{\varepsilon}, \mathcal{S}_{0}\right)$ such that:

$$
\left\{\begin{array}{l}
u=Q \text { in } \Omega_{\varepsilon} \\
u=Q \text { on } \partial \mathcal{N}_{\varepsilon}^{\mathcal{T}} \\
\|\nabla u\|_{L^{2}\left(\Omega_{\varepsilon} \cup \mathcal{N}_{\varepsilon}^{\mathcal{T}}\right)} \leq c\|\nabla Q\|_{L^{2}\left(\Omega_{\varepsilon}\right)}
\end{array}\right.
$$

Let $\Omega_{\varepsilon}^{\prime}=\Omega_{\varepsilon} \cup \mathcal{N}_{\varepsilon}^{\mathcal{T}}$. We want now to construct a function $v: \mathcal{N}_{\varepsilon}^{\mathcal{S}} \rightarrow \mathcal{S}_{0}$ such that $v=u$ on $\partial \Omega_{\varepsilon}^{\prime}$ and that there exists a constant $c>0$, independent of $\varepsilon$ such that $\|\nabla v\|_{L^{2}\left(\mathcal{N}_{\mathcal{E}}^{\mathcal{S}}\right)} \leq c\|\nabla u\|_{L^{2}\left(\Omega_{\varepsilon}^{\prime}\right)}$.

But in the case of the family $\mathcal{N}_{\varepsilon}^{\mathcal{S}}$, these "inner parallelepipeds" are pairwise disjoint for $\varepsilon$ small enough, therefore we can construct $v$ in each $\mathcal{C}_{\varepsilon}^{i}$, for every $i \in \overline{1, N_{\varepsilon}}$ and control, independent of $\varepsilon$, $\|\nabla v\|_{L^{2}\left(\mathcal{C}_{\varepsilon}^{i}\right)}$ with $\|\nabla u\|_{L^{2}\left(\mathcal{P}_{\varepsilon}^{i}\right)}$, where $\mathcal{R}_{\varepsilon}^{i}$ is the "surrounding" box for $\mathcal{C}_{\varepsilon}^{i}$, constructed in the same way as $\mathcal{R}_{\varepsilon}^{z, m}$.

Lemma A.6. Let $a, b \in \mathbb{R}_{+}^{*}$ with $a<b$, let $\mathcal{B}_{a}=\left\{x \in \mathbb{R}^{3}|| x \mid<a\right\}$ and let $\mathcal{A}_{a, b}=\mathcal{B}_{b} \backslash \overline{\mathcal{B}_{a}}$. Let $u \in H^{1}\left(\mathcal{A}_{1,2}, \mathcal{S}_{0}\right)$. Then the function $v: \mathcal{B}_{1} \rightarrow \mathcal{S}_{0}$ defined as

$$
v(x, y, z)=\left\{\begin{array}{l}
\varphi\left(\sqrt{x^{2}+y^{2}+z^{2}}\right) u\left(\left(\frac{2}{\sqrt{x^{2}+y^{2}+z^{2}}}-1\right)(x, y, z)\right) \\
\quad+\left(1-\varphi\left(\sqrt{x^{2}+y^{2}+z^{2}}\right)\right) f_{\mathcal{A}_{1,3 / 2}} u(\xi, \eta, \tau) \mathrm{d}(\xi, \eta, \tau), \text { for } \frac{1}{2} \leq \sqrt{x^{2}+y^{2}+z^{2}}<1 \\
f_{\mathcal{A}_{1,3 / 2}} u(\xi, \eta, \tau) \mathrm{d}(\xi, \eta, \tau), \text { for } 0 \leq \sqrt{x^{2}+y^{2}+z^{2}} \leq \frac{1}{2}
\end{array}\right.
$$


is from $H^{1}\left(\mathcal{B}_{1}, \mathcal{S}_{0}\right)$, where $\varphi \in C_{c}^{\infty}\left(\left(\frac{1}{2}, \frac{3}{2}\right)\right)$ is the following bump function defined as

$$
\varphi(\rho)=\left\{\begin{array}{l}
\exp \left\{4-\frac{4}{(2 \rho-1)(3-2 \rho)}\right\}, \forall \rho \in\left(\frac{1}{2}, \frac{3}{2}\right), \\
0, \forall \rho \in \mathbb{R} \backslash\left(\frac{1}{2}, \frac{3}{2}\right)
\end{array},\right.
$$

the product $\varphi(\rho)$ u represents product between a scalar and a $Q$-tensor and $f$ represents the average integral sign. Moreover, there exists a constant $c>0$ such that: $\left\|v_{t}\right\|_{L^{2}\left(\mathcal{B}_{1}\right)} \leq c\left\|u_{t}\right\|_{L^{2}\left(\mathcal{A}_{1,3 / 2}\right)}$ for any $t \in\{x, y, z\}$, where $v_{t}$ represents the partial derivative of $v$ with respect to $t$, and:

$$
\|\nabla v\|_{L^{2}\left(\mathcal{B}_{1}\right)} \leq c\|\nabla u\|_{L^{2}\left(\mathcal{A}_{1,3 / 2}\right)} .
$$

Remark A.7. Lemma A.6 is just a different version of Lemma A.5. The proof follows the same steps as in Lemma A.5.

Now if we use instead of $T_{2}$ the transformation $\Lambda_{3}$, from [17], which is a bi-Lipschitz mapping that transforms the unit ball into the unit cube, and then the transformations $T_{3}$ and $T_{4}$ as before, we end up with the function $v$ being an extension of $u$ that satisfies:

$$
\left\{\begin{array}{l}
v \in H^{1}\left(\mathcal{C}_{\varepsilon}^{i}\right) \\
v=u \text { on } \partial \mathcal{C}_{\varepsilon}^{i} \\
\|\nabla v\|_{L^{2}\left(\mathcal{C}_{\varepsilon}^{i}\right)} \leq c\|\nabla u\|_{L^{2}\left(\mathcal{R}_{\varepsilon}^{i}\right)}
\end{array}\right.
$$

Because the objects $\mathcal{R}_{\varepsilon}^{i}$ are pairwise disjoint for $\varepsilon$ small enough, we construct therefore an extension $v \in$ $H^{1}\left(\Omega, \mathcal{S}_{0}\right)$ of $u \in H^{1}\left(\Omega_{\varepsilon}^{\prime}, \mathcal{S}_{0}\right)$ such that:

$$
\left\{\begin{array}{l}
v=u \text { in } \Omega_{\varepsilon}^{\prime} \Rightarrow v=Q \text { in } \Omega_{\varepsilon} \text { and } v=Q \text { on } \partial \mathcal{N}_{\varepsilon}^{\mathcal{T}} \\
v=u \text { on } \partial \mathcal{N}_{\varepsilon}^{\mathcal{S}} \Rightarrow v=Q \text { on } \partial \mathcal{N}_{\varepsilon}^{\mathcal{S}} \\
\|\nabla v\|_{L^{2}(\Omega)} \leq c\|\nabla u\|_{L^{2}\left(\Omega_{\varepsilon}^{\prime}\right)} \leq \tilde{c}\|\nabla Q\|_{L^{2}\left(\Omega_{\varepsilon}\right)}
\end{array}\right.
$$

So we have $v \in H^{1}(\Omega), v=Q$ in $\Omega_{\varepsilon}, v=Q$ on $\partial \mathcal{N}_{\varepsilon}$ and there exists an $\varepsilon$-independent constant such that:

$$
\|\nabla v\|_{L^{2}(\Omega)} \leq c\|\nabla Q\|_{L^{2}\left(\Omega_{\varepsilon}\right)} .
$$

\section{A.4 Integrated energy densities}

In this subsection, we present two propositions that are used in order to prove that using relation (3.2), that is:

$$
f_{\text {hom }}(Q)=\frac{2}{p} \int_{\partial \mathcal{C}} f_{s}(Q, \nu) \mathrm{d} \sigma
$$

then by using, for example, the choice of the surface energy density defined in (3.8), which is:

$$
f_{s}^{L D G}(Q, \nu)=\frac{p}{4}\left(\left(a^{\prime}-a\right)\left(\nu \cdot Q^{2} \nu\right)-\left(b^{\prime}-b\right)\left(\nu \cdot Q^{3} \nu\right)+2\left(c^{\prime}-c\right)\left(\nu \cdot Q^{4} \nu\right)\right),
$$


we can obtain the corresponding homogenised functional defined in (3.9), that is:

$$
f_{\text {hom }}^{L D G}(Q)=\left(a^{\prime}-a\right) \operatorname{tr}\left(Q^{2}\right)-\left(b^{\prime}-b\right) \operatorname{tr}\left(Q^{3}\right)+\left(c^{\prime}-c\right)\left(\operatorname{tr}\left(Q^{2}\right)\right)^{2} .
$$

More specifically, Proposition A.8 treats the case of the classical quartic polynomial in the scalar invariants of $Q$ for the bulk energy, defined in (1.2), where the choice of the surface energy density is in (3.8), and the more general version of it, defined in (3.4), with the surface energy density defined in (3.16). Both cases have all of the terms from the picked surface energy densities of the form $\nu \cdot Q^{k} \nu$, with $k \geq 2$. Proposition A.9 treats only the Rapini-Papoular case, where the surface energy density is defined in (3.12).

Proposition A.8. For any $k \in \mathbb{N}, k \geq 2$ and for a fixed matrix $Q \in \mathcal{S}_{0}$, we have:

$$
\operatorname{tr}\left(Q^{k}\right)=\frac{1}{2} \int_{\partial \mathcal{C}}\left(\nu \cdot Q^{k} \nu\right) \mathrm{d} \sigma
$$

where $\partial \mathcal{C}$ is defined in (2.3) and $\nu$ is the exterior unit normal to $\partial \mathcal{C}$.

Proof. Let $Q^{k}=\left(\begin{array}{lll}q_{11, k} & q_{12, k} & q_{13, k} \\ q_{21, k} & q_{22, k} & q_{23, k} \\ q_{13, k} & q_{32, k} & q_{33, k}\end{array}\right)$. According to $(2.3)$, we have $\partial \mathcal{C}=\mathcal{C}^{x} \cup \mathcal{C}^{y} \cup \mathcal{C}^{z}$. We compute first the intergral for $\mathcal{C}^{x}$, on which $\nu=( \pm 1,0,0)^{T}$ :

$$
\int_{\mathcal{C}^{x}}\left(\nu \cdot Q^{k} \nu\right) \mathrm{d} \sigma=\int_{\mathcal{C}^{x}}\left(( \pm 1,0,0)^{T} \cdot\left( \pm q_{11, k}, \pm q_{21, k}, \pm q_{31, k}\right)^{T}\right) \mathrm{d} \sigma=\int_{\mathcal{C}^{x}} q_{11, k} \mathrm{~d} \sigma=2 q_{11, k}
$$

since $\mathcal{C}$ has length 1.

In the same way, we obtain:

$$
\int_{\mathcal{C}^{y}}\left(\nu \cdot Q^{k} \nu\right) \mathrm{d} \sigma=2 q_{22, k} \quad \text { and } \quad \int_{\mathcal{C}^{z}}\left(\nu \cdot Q^{k}\left(x_{0}\right) \nu\right) \mathrm{d} \sigma=2 q_{33, k},
$$

from which we obtain

$$
\int_{\partial \mathcal{C}}\left(\nu \cdot Q^{k} \nu\right) \mathrm{d} \sigma=2 \operatorname{tr}\left(Q^{k}\right)
$$

For the Rapini-Papoular case, we prove that:

Proposition A.9. For a fixed matrix $Q \in \mathcal{S}_{0}$, we have:

$$
6 \operatorname{tr}\left(Q^{2}\right)+4=\int_{\partial \mathcal{C}} \operatorname{tr}\left(Q-Q_{\nu}\right)^{2} \mathrm{~d} \sigma
$$

where $\partial \mathcal{C}$ is defined in (2.3), $Q_{\nu}=\nu \otimes \nu-\mathbb{I}_{3} / 3, \nu$ represents the exterior unit normal to $\partial \mathcal{C}$ and $\mathbb{I}_{3}$ is the $3 \times 3$ identity matrix.

Proof. First of all, we can see that $\operatorname{tr}\left(Q-Q_{\nu}\right)^{2}=\operatorname{tr}\left(Q^{2}\right)-\operatorname{tr}\left(Q Q_{\nu}\right)-\operatorname{tr}\left(Q_{\nu} Q\right)+\operatorname{tr}\left(Q_{\nu}^{2}\right)$. 


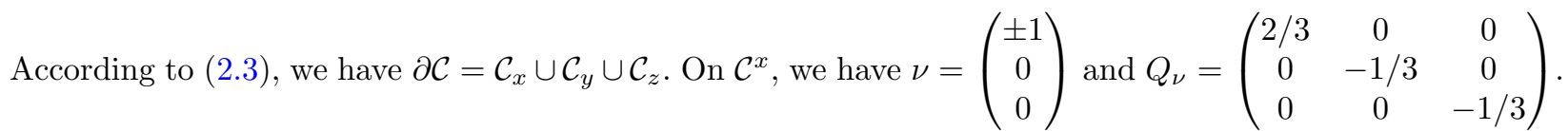
Then $\operatorname{tr}\left(Q_{\nu}^{2}\right)=\left(\frac{2}{3}\right)^{2}+\left(-\frac{1}{3}\right)^{2}+\left(-\frac{1}{3}\right)^{2}=\frac{2}{3}$. We also obtain $\operatorname{tr}\left(Q_{\nu}^{2}\right)=\frac{2}{3}$ on $\mathcal{C}^{y}$ and $\mathcal{C}^{z}$. Therefore we obtain:

$$
\int_{\partial \mathcal{C}} \operatorname{tr}\left(Q_{\nu}^{2}\right) \mathrm{d} \sigma=6 \cdot \frac{2}{3}=4
$$

where the constant 6 comes from the total surface of the cube $\mathcal{C}$.

Let $Q=\left(\begin{array}{ccc}q_{11} & q_{12} & q_{13} \\ q_{12} & q_{22} & q_{23} \\ q_{13} & q_{23} & -q_{11}-q_{22}\end{array}\right)$. Using the computations done earlier for $Q_{\nu}$ on $\mathcal{C}^{x}, \mathcal{C}^{y}$ and $\mathcal{C}^{z}$, we get:

$$
\begin{aligned}
& \int_{\mathcal{C}^{x}}\left(\operatorname{tr}\left(Q_{\nu} Q\right)+\operatorname{tr}\left(Q Q_{\nu}\right)\right) \mathrm{d} \sigma=2\left(\frac{2 q_{11}}{3}-\frac{q_{22}}{3}+\frac{q_{11}+q_{22}}{3}\right)=2 q_{11} \\
& \int_{\mathcal{C}^{y}}\left(\operatorname{tr}\left(Q_{\nu} Q\right)+\operatorname{tr}\left(Q Q_{\nu}\right)\right) \mathrm{d} \sigma=2\left(-\frac{q_{11}}{3}+\frac{2 q_{22}}{3}+\frac{q_{11}+q_{22}}{3}\right)=2 q_{22} \\
& \int_{\mathcal{C}^{z}}\left(\operatorname{tr}\left(Q_{\nu} Q\right)+\operatorname{tr}\left(Q Q_{\nu}\right)\right) \mathrm{d} \sigma=2\left(-\frac{q_{11}}{3}-\frac{q_{22}}{3}-\frac{2 q_{11}+2 q_{22}}{3}\right)=-2 q_{11}-2 q_{22} .
\end{aligned}
$$

Combining the last three relations, we get that

$$
\int_{\partial \mathcal{C}}\left(\operatorname{tr}\left(Q_{\nu} Q\right)+\operatorname{tr}\left(Q Q_{\nu}\right)\right) \mathrm{d} \sigma=0
$$

from which the conclusion follows, with the observation that the constant 6 in front of $\operatorname{tr}\left(Q^{2}\right)$ appears from the total surface of the cube $\mathcal{C}$, which has the length equal to 1.

Remark A.10. The constant 4 from Proposition A.9 is neglected when we are studying the asymptotic behaviour of the minimisers of the functional (3.13), since adding constants do not influence the form and the existence of the possible minimisers.

Acknowledgements. The work of Razvan-Dumitru Ceuca is supported by the Spanish Ministry of Economy and Competitiveness MINECO through BCAM Severo Ochoa excellence accreditation SEV-2013-0323-17-1 (BES-2017-080630) and through project MTM2017-82184-R funded by (AEI/FEDER, UE) and acronym "DESFLU". The author would like to thank Jamie Taylor and Giacomo Canevari for insightful discussions that have benefited this work and for their support granted during the making of it. The author would also like to thank his Ph.D. supervisors, Arghir-Dani Zarnescu and Eugen Varvaruca, for the constant mathematical and moral support offered during the proccess of generating this work. In the end, the author would like to thank the anonymous reviewers for their careful and critical reading of this manuscript, providing insightful and helpful comments and suggestions.

\section{REFERENCES}

[1] R. Adams and J. Fournier, Sobolev Spaces. Academic Press, London (2003).

[2] S. Alama, L. Bronsard and X. Lamy, Minimizers of the Landau-de Gennes energy around a spherical colloid particle. Arch. Ratl. Mech. Anal. 222 (2016) 427-450.

[3] S. Alama, L. Bronsard and X. Lamy, Spherical particle in nematic liquid crystal under an external field: the Saturn ring regime. J. Nonlinear Sci. 2018 (2018) 1-23.

[4] T. Baldacchini, Three-Dimensional Microfabrication Using Two-Photon Polymerization. 1st Edition. Elsevier (2015). 
[5] T.P. Bennett, G. D'Alessandro and K.R. Daly, Multiscale models of colloidal dispersion of particles in nematic liquid crystals. Phys. Rev. E 90 (2014) 062505.

[6] L. Berlyland, D. Cioranescu and D. Golovaty, Homogenization of Ginzburg-Landau model for a nematic liquid crystal with inclusions. J. de mathematiques pures et appliquées 84 (2016) 97-136.

[7] M. Buscaglia, T. Bellini, C. Chiccoli, F. Mantegazza, P. Pasini, M. Rotunno and C. Zannoni, Phys. Rev. E 74 (2006) 011706.

[8] M.C. Calderer, A. DeSimone, D. Golovaty and A. Panchenko, An effective model for nematic liquid crystal composites with ferromagnetic inclusions. SIAM J. Appl. Math. 74 (2014) 237-262.

[9] G. Canevari, M. Ramaswamy and A. Majumdar, Radial symmetry on three-dimensional shells in the Landau-de Gennes theory. Physica D 314 (2016) 18-34.

[10] G. Canevari, A. Segatti and M.M. Veneroni, Morse's index formula in VMO on compact manifold with boundary. J. Funct. Anal. 269 (2015) 3043-3082.

[11] G. Canevari and A. Segatti, Defects in Nematic Shells: a $\Gamma$-convergence discrete-to-continuum approach. Arch. Ratl. Mech. Anal. 229 (2018) 125-186.

[12] G. Canevari and A. Segatti, Variational analysis of nematic shells. Trends in Applications of Mathematics to Mechanics. Springer-INdAM series 27 (2018) 81-102.

[13] G. Canevari and A.D. Zarnescu, Design of effective bulk potentials for nematic liquid crystals via colloidal homogenisation. Math. Models Methods Appl. Sci. (2019) 10.1142/S0218202520500086.

[14] G. Canevari and A.D. Zarnescu, Polydispersity and surface energy strength in nematic colloids. Math. Eng. 2 (2020) $290-312$.

[15] D. Cioranescu and P. Donato, An introduction to homogenization. Oxford Lecture Series in Mathematics and Its Applications (1999).

[16] P.G. De Gennes and J. Prost, The Physics of Liquid Crystals. International series of monographs on physics. Clarendon Press (1993).

[17] J. Griepentrog, W. Höppner, H.-C. Kaiser and J. Rehberg, A bi-Lipschitz continuous, volume preserving map from the unit ball onto a cube. Note di Matematica 28 (2008) 177-193.

[18] D. Jayasri, M. Ravnik and Š. Žumer, Shape tuning the colloidal assemblies in nematic liquid crystals. Soft Matter. 8 (2012) 1657.

[19] L. Longa, D. Montelesan and H.R. Trebin, An extension of the Landau-Ginzburg-de Gennes theory for liquid crystals. Liquid Crys. 2 (1987).

[20] N.J. Mottram and C. Newton, Introduction to Q-tensor theory (2014). Preprint arXiv:1409.3542.

[21] I. Muševič, M. Škarabot, U. Tkalec, M. Ravnik and Š. Žumer, Two-dimensional nematic colloidal crystals self-assembled by topological defects. Science $\mathbf{3 1 3}$ (2006) 954.

[22] M. Ravnik, M. Škarabot, Š. Žumer, U. Tkalec, I. Poberaj, D. Babič, N. Osterman and I. Muševič. Entangled Nematic Colloidal Dimers and Wires. Phys. Rev. Lett. 99 (2007) 247801.

[23] F. Serra, S. Eaton, R. Cerbino, M. Buscaglia, G. Cerullo, R. Osellame and T. Bellini, Liquid crystals: nematic liquid crystals embedded in cubic microlattices: memory effects and bistable pixels (Adv. Funct. Mater. 32/2013). Adv. Funct. Mater. 23 (2013) 3990.

[24] F. Serra, K.C. Vishnubhatla, M. Buscaglia, R. Cerbino, R. Osellame, G. Cerullo and T. Bellini, Soft Matter 7 (2011) 10945.

[25] Y. Wang, G. Canevari and A. Majumdar, Order reconstruction for nematics on squares with isotropic inclusions: a Landau-de Gennes study. Preprint arXiv:1803.02597 (2018).

[26] W.P. Ziemer, Weakly differentiable functions. Springer-Verlag, New York (1989).

\section{Subscribe to Open (S2O) A fair and sustainable open access model}

This journal is currently published in open access under a Subscribe-to-Open model (S2O). S2O is a transformative model that aims to move subscription journals to open access. Open access is the free, immediate, online availability of research articles combined with the rights to use these articles fully in the digital environment. We are thankful to our subscribers and sponsors for making it possible to publish this journal in open access, free of charge for authors.

\section{Please help to maintain this journal in open access!}

Check that your library subscribes to the journal, or make a personal donation to the S2O programme, by contacting subscribers@edpsciences.org

More information, including a list of sponsors and a financial transparency report, available at: https://www.edpsciences.org/en/maths-s2o-programme 